\title{
Classificação dos estados cognitivos orientados pelo sujeito baseada na variabilidade da frequência cardíaca
}

\author{
Carlos Enrique Paucar Farfán \\ DISSERTAÇÃO APRESENTADA \\ $\mathrm{AO}$ \\ Instituto DE MATEMÁticA E EstatísticA \\ DA \\ UNIVERSIDADE DE SÃO PAULO \\ PARA \\ OBTENÇÃO DO TÍTULO \\ $\mathrm{DE}$ \\ Mestre EM CiÊnCIAS
}

\author{
Programa de Pós-Graduação em Ciência da Computação \\ Orientador: Prof. Dr. André Fujita
}

Durante o desenvolvimento deste trabalho o autor recebeu auxílio financeiro da

CAPES(88887.200887/2018-00) e HP-Lab

São Paulo, Junho de 2021 


\title{
Classificação dos estados cognitivos orientados pelo sujeito baseada
} na variabilidade da frequência cardíaca

\author{
Esta versão da dissertação contém as correções e alterações sugeridas \\ pela Comissão Julgadora durante a defesa da versão original do trabalho, \\ realizada em 28/05/2021. Uma cópia da versão original está disponível no \\ Instituto de Matemática e Estatística da Universidade de São Paulo.
}

Comissão Julgadora:

- Prof. Dr. André Fujita (orientador) - IME-USP

- Prof. Dr. Alexandre Alarcon Steiner - ICB-USP

- Prof. Dr. Daniel Yasumasa Takahashi - ICe-UFRN 
Autorizo a reprodução e divulgação total ou parcial deste trabalho, por qualquer meio convencional ou eletrônico, para fins de estudo e pesquisa, desde que citada a fonte. 


\section{Agradecimentos}

Passar pela USP foi uma experiência de muito aprendizagem, onde tive a possibilidade de conhecer muitos amigos que me ajudaram no meu crescimento acadêmico, profissional e pessoal, portanto quero agradecer ao pessoal que fui conhecendo em ordem cronológica. Meu orientador o Professor André Fujita que me aceito para trabalhar com ele no seu grupo, a meus colegas do grupo Fujita's Team: a Suzana, a Taiane, o Maciel, o Grover, o Edu , o Diego, o Guillerme, o Lucas, o Carlos Relvas e a Juliana.

Meus colegas com os que entrei na pós-graduação: o Luis Araujo, a Tathiane Rosa, a Thamillys e especialmente ao Samuel Plaça de Paula de quem aprendi muito nas matérias de teoria do IME e agradeço pela paciência que teve comigo.

Meus colegas do Laboratório de Bioinfo: o Hans, a Nury, a Evelyn, o Carlos Eduardo, o Miguel, a Candy, o Christian e o Reynaldo.

Ao grupo de ARGUSP(Autotuning Research Group - USP) dirigida pelo Professor Goldman e especialmente agradecer ao Pedro Henrique Rocha Bruel pelas dicas e sugestões que me ajudaram e motivaram na pesquisa.

Meus amigos da moradia: o Clayton, o Eric, o Angelo, o Raul, o Rodrigo, a Lauriceia, o Oscar, a Vanesa e o Demba.

Também a todos os que participaram na coleta de dados de minha pesquisa. Agradeço à Katia, secretária da CCP. Sou muito grato aos professores do Departamento de Computação do IMEUSP que me encorajaram a terminar este projeto, em particular ao professor Roberto Hirata quem me recebeu no inicio do mestrado e aos professores da Poli-USP em particular ao Professor Kechi Hirama que me deu a oportunidade de ser monitor nas aulas de Laboratório de Software.

Agradeço à CAPES e à HP-Lab pelo financiamento durante o desenvolvimento do projeto. Também agradeço à Comissão Nacional de Ética em Pesquisa (CONEP) pela autorização para continuar com a pesquisa.

Tudo o acontecido no mestrado não teria sido possível se não for por uma causa, minha mãe a Senhora Sofía Jacinta Farfán Zevallos que me deu o empurrão e a motivação para continuar nos estudos.

Finalmente agradeço a todos os leitores deste projeto. 


\section{Resumo}

Farfán, C. E. P. Classificação dos estados cognitivos orientados pelo sujeito baseada na variabilidade da frequência cardíaca.2021. Dissertação (Mestrado em Ciência da Computação - Instituto de Matemática e Estatística, Universidade de São Paulo, São Paulo, 2021.

A decodificação cerebral ganhou muita atenção durante as últimas décadas. Os primeiros estudos foram baseados em eventos discretos. Recentemente, foram desenvolvidos métodos para decodificar estados cognitivos mais contínuos e puramente subjetivos. Entretanto, todos eles requerem dispositivos caros, difíceis de manusear e desconfortáveis para o uso diário. Aqui propomos uma alternativa baseada na frequência cardíaca (FC). Já é bem conhecido que podemos discriminar algumas atividades físicas com base na FC. Entretanto, a questão não intuitiva é: podemos discriminar tarefas cognitivas com base na FC? Submetemos 25 sujeitos a quatro tarefas cognitivas: descansar em silêncio, lembrar dos eventos do dia anterior, cantar e subtrair números. Coletamos o eletrocardiograma duas vezes para cada indivíduo, separados por aproximadamente uma semana. Para coletar a FC, usamos uma faixa de sensor de tórax comercial utilizada por atletas. Treinamos uma máquina vetorial de suporte usando os dados coletados no primeiro dia. Depois a validamos no conjunto de dados coletados no segundo dia. Nossos resultados mostram precisão mais significativa do que o que esperamos ao acaso $(p<0,001)$. Dependendo do indivíduo e do conjunto de tarefas cognitivas, obtivemos quase $100 \%$ de acurácia. Também verificamos o potencial da FC de ser um biomarcador para identificar o indivíduo (acurácia de aproximadamente $18 \%, \mathrm{p}=0,001$ ). Assim, concluímos que a FC apresenta informações sobre os estados mentais.

Palavras-chave: Eletrocardiograma, Variabilidade da frequência cardíaca, Estado cognitivo, Classificação, Biomarcador. 


\section{Abstract}

\section{Farfán, C. E. P. Classification of Subject-Driven Cognitive States Based on HeartRate}

Variability.2021. Dissertação (Mestrado em Ciência da Computação - Instituto de Matemática e Estatística, Universidade de São Paulo, São Paulo, 2021.

Brain decodification has gained much attention over the last decades. The first studies were based on discrete events. Recently, methods for decoding more continuous and purely subject-driven cognitive states have been developed. However, all of them require expensive, difficult to handle, and uncomfortable devices for daily use. Here we propose an alternative based on heart rate (HR). It is already well known that we can discriminate some physical activities based on HR. However, the not intuitive question is, can we discriminate cognitive tasks based on HR? We submitted 25 subjects to four cognitive tasks: resting quietly, remembering their day's events, singing lyrics, or subtracting numbers. We collected the electrocardiogram twice for each individual, separated by approximately one week. To collect the HR, we used a commercial chest sensor band used by athletes. We trained a support vector machine using data collected on day one. Then we validated it in the dataset collected on day two. Our results show accuracy more significant than what we expect at random $(p<0.001)$. Depending on the individual and set of cognitive tasks, we obtained almost $100 \%$ accuracy. We also verified the HR's potential to be a biomarker to identify the individual (accuracy of approximately $18 \%, p=0.001)$. Altogether, we conclude the HR presents mental states' information and can help for daily use in the future.

Keywords: Electrocardiogram, Heart rate variability, Cognitive state, Classification, Biomarker. 


\section{Sumário}

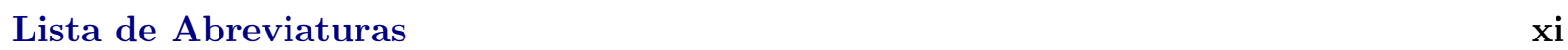

Lista de Figuras $\quad$ xiii

Lista de Tabelas $\quad$ xv

1 Introdução $\quad 1$

1.1 Considerações Preliminares . . . . . . . . . . . . . . . . . . . . 2

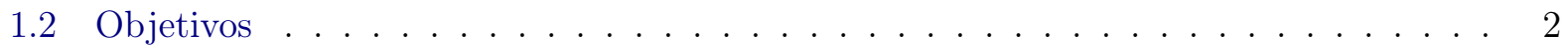

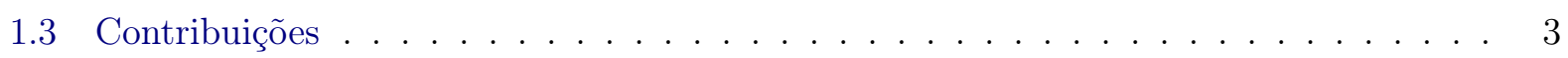

1.4 Motivação . . . . . . . . . . . . . . . . . . . . . . . . 3

1.5 Organização do Trabalho . . . . . . . . . . . . . . . . . . . . . 3

2 Conceitos $\quad \mathbf{5}$

2.1 Fundamentos básicos do sinal ECG . . . . . . . . . . . . . . . . . . . 5

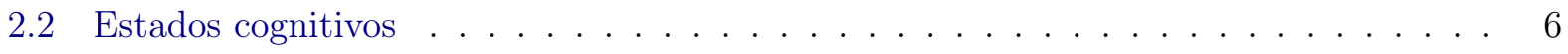

2.3 Decodificação de atividades cerebrais . . . . . . . . . . . . . . . . . . 6

2.4 Hardware selecionado . . . . . . . . . . . . . . . . . . . . 8

2.5 Variabilidade de Frequência Cardíaca . . . . . . . . . . . . . . . . . . . 8

2.5.1 Métricas no domínio do tempo . . . . . . . . . . . . . . . . 9

2.5.2 Métricas no domínio da frequência . . . . . . . . . . . . . . . . . . . . 11

2.5.3 Métricas não lineares . . . . . . . . . . . . . . . . . . . . . . . . . 11

2.6 Variáveis que influenciam ao HRV . . . . . . . . . . . . . . . . . . . . . . 12

2.7 Classificadores de Aprendizagem de Máquina . . . . . . . . . . . . . . . . . . 12

2.7 .1 Máquinas de Vetores de Suporte . . . . . . . . . . . . . . . . . . . . . . . . 12

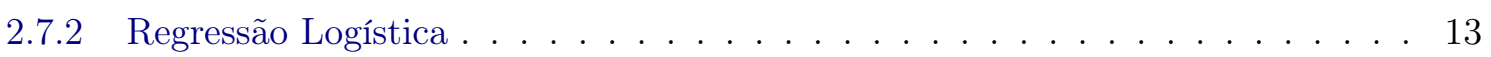

2.7 .3 Árvores de Decisão . . . . . . . . . . . . . . . . . . . . . . . . . . . . . . . 14

2.7 .4 Florestas Aleatórias . . . . . . . . . . . . . . . . . 15

2.8 Métricas de desempenho do classificador . . . . . . . . . . . . . . . . . . . . 15

2.9 Otimização de hiperparâmetros . . . . . . . . . . . . . . . . . . . . . . 16

2.10 Importância das características . . . . . . . . . . . . . . . . . . . . 17

2.11 Desenho de Experimentos . . . . . . . . . . . . . . . . . 18

2.11 .1 Desenhos Fatoriais Completos . . . . . . . . . . . . . . . . . . . 18

2.11 .2 Desenhos Fatoriais Fracionários . . . . . . . . . . . . . . . . 18

2.11 .3 Desenhos Aleatórios . . . . . . . . . . . . . . . . . . . . . . . . . 18 
2.11.4 Desenho Plackett Burman . . . . . . . . . . . . . . . . . . . . . . . . 19

2.11 .5 Desenhos Ótimos . . . . . . . . . . . . . . . . . . . . . . . 19

2.11.6 Abordagem de exploração do desenho de experimentos . . . . . . . . . . . . . 20

3 Coleta de Dados e Pré-processamento 22

3.1 Coleta de Dados . . . . . . . . . . . . . . . . . . . . . . . 22

3.1.1 Aplicativo de coleta de dados . . . . . . . . . . . . . . . . . . . . 23

3.1 .2 Protocolo de coleta de dados . . . . . . . . . . . . . . . . . . . . . 24

3.2 Carregamento e observação dos dados . . . . . . . . . . . . . . . . . . 25

3.3 Filtragem dos dados . . . . . . . . . . . . . . . . . . 26

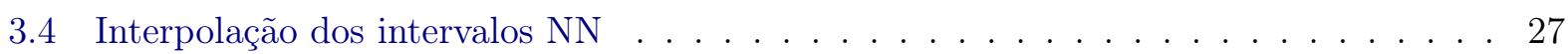

3.5 Cálculo das métricas da HRV . . . . . . . . . . . . . . . . . . . . . . 29

4 Experimentos e análise de resultados $\quad 31$

4.1 Modelagem do classificador . . . . . . . . . . . . . . . . . . . . 31

4.1 .1 Normalização dos dados . . . . . . . . . . . . . . . . . . . . . . . . 31

4.1 .2 Casos de análise . . . . . . . . . . . . . . . . . . . 31

4.2 Especificação de espaço de busca de hiperparâmetros . . . . . . . . . . . . . . . . 33

4.3 Desempenho no ajuste de hiperparâmetros . . . . . . . . . . . . . . . . . 35

4.4 Desempenho na seleção de métricas . . . . . . . . . . . . . . . . . . . . 35

4.5 Desempenho de ajuste de hiperparâmetros com seleção de métricas . . . . . . . . . . 37

4.6 Classificação dos participantes com base no sinal cardíaco . . . . . . . . . . . . . . 39

5 Discussão de Resultados e Conclusões $\quad 41$

$\begin{array}{ll}\text { A Banco de Dados } & 45\end{array}$

B Comitê de Ética em Pesquisa $\quad 45$

Referências Bibliográficas $\quad 50$ 


\section{Lista de Abreviaturas}

ANS: Autonomous nervous system

BOLD: Blood oxygen level dependent imaging

DOE: Design of Experiments

DT: Decision Tree

ECG: Electrocardiogram

ECoG: Electrocorticogram

EEG: Electroencephalogram

fMRI: Functional magnetic resonance imaging

fNIRS: Functional near-infrared spec-troscopy

GUI: Grafical User Interface

HCI: Human Computer Interaction

HF: High Frequency

HR: Heart Rate

HRV: Heart Rate Variability

LF: Low Frequency

LR: Logistic Regression

ML: Machine Learning

pNN50: Percentage of successive NN intervals $>50 \mathrm{~ms}$

PNS: Parasympathetic Nervous System

RF: Random Forest

rMSSD: root-Mean-Square of the Successive NN interval Difference

SDANN: Standard Deviation of the averaged NN intervals for all 5-min segments

SDNN index: mean of the standard deviations of all NN intervals for all 5-min segments

SDNN: Standard Deviation of all NN intervals over $24 \mathrm{~h}$

SNS: Sympathetic Nervous System

SVM: Support Vector Machine

ULF: Ultra Low Frequency

VLF: Very Low Frequency 


\section{Lista de Figuras}

2.1 Comportamentos das ondas de ECG em estados de ativação(despolarização) e recuperação(repolarização) dos átrios e ventrículos do coração. ${ }^{1} \ldots$. . . . . . . . . . 5

2.2 Variabilidade no intervalo entre batimentos. O eixo $X$ representa o tempo em segundos e o eixo $Y$ representa a amplitude do sinal em milivolts. ${ }^{2} \ldots \ldots \ldots$

2.3 Especificidade do domínio da cognição e exemplos de processos cognitivos subjacentes a esses mecanismos. Figura Adaptada ${ }^{3}$. . . . . . . . . . . . . . . . . . 7

2.4 Gráficos de correlação do sensor de frequência cardíaca Polar H7 vs ECG durante respiração guiada e normal da métrica rMSSD. A linha preta sólida representa a linha de equivalência $(r=1,0)$. Na parte inferior do gráfico esta o Erro Técnico de Medição (TEM, do inglês Technical Error of Measurement) dos 29 participantes avaliados. Adaptado de [PSA $\left.{ }^{+} 17\right]$.

2.5 A relação entre a frequência cardíaca (HR) e o intervalo R-R (RRi) presenta um comportamento hiperbólico; uma variação no $\mathrm{RRi}(\Delta \mathrm{RRi})$ resulta em mudanças marcadamente diferentes no HR $(\Delta \mathrm{HR})$. Adaptado de $[\mathrm{DT} 16] \ldots \ldots \ldots$

2.6 Matriz de confusão. . . . . . . . . . . . . . . . . . . . . . . . . . . . . . 16

3.1 Diagrama do pipeline do desenvolvimento do trabalho. . . . . . . . . . . . . . 22

3.2 Configuração do experimento. (A) Registro de sinais cardíacos com o sensor Polar H10; (B) Registro de vídeo do comportamento dos usuários,ações observáveis dos usuários (expressão facial, postura e gestos) e (C) Registro de vídeo do conteúdo atual da tela. . . . . . . . . . . . . . . . . . . . . . 23

3.3 Aplicativo de Coleta de Dados com as séries temporais das métricas da HRV dos últimos 45 dados recebidos do sensor com as opções para o usuário registrar uma sessão de atividade. . . . . . . . . . . . . . . . . . . . . . . 24

3.4 Média da HR e dos intervalos NN de todos os participante nas diferentes atividades cognitivas em cada tarefa. . . . . . . . . . . . . . . . . . . 25

3.5 Serie do tempo dos intervalos NN para um participante nas diferentes atividades

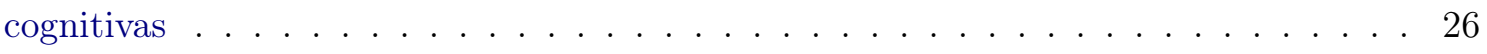

3.6 Espectro da potencia do sinal dos intervalos NN mostrando as bandas de frequência (VLF, LF e HF) para sessões de 10 minutos em diferentes dias. . . . . . . . . . . . . 27

3.7 Método do Malik para encontrar valores ectópicos nos intervalos RR . . . . . . . 28

3.8 (A) Intervalos RR na tarefa cognitiva de lembrança e (B) o filtro e interpolação aplicado na serie (A) para obter os Intervalos NN na tarefa cognitiva de lembrança, as series tem um tempo de coleta de 10 min. . . . . . . . . . . . . . . . 28 
3.9 Interpolação por janelas deslizantes sobre a serie dos intervalos NN, com um deslocamento de tamanho $d$ para janelas de tamanho fixo, para depois calcular as métricas da HRV por cada janela. . . . . . . . . . . . . . . . . . . . . . . 28

4.1 Acurácias de classificação de tarefas cognitivas intradiárias para o SVM. (A) Obtivemos a acurácia da classificação usando a primeira metade dos dados do dia 1 como o conjunto de treinamento e a segunda metade como o conjunto de validação. (B) Obtivemos a acurácia da classificação usando a primeira metade dos dados do dia 2 como o conjunto de treinamento e a segunda metade como o conjunto de validação.

4.2 Acurácias de classificação de tarefas cognitivas interdiárias. Usamos os dados do dia 1 como o conjunto de treinamento e os dados do dia 2 como o conjunto de validação.

4.3 Importância das métricas nos diferentes casos de análise interdiarios . . . . . . . . . 36

4.4 Comparação da acurácia média dos modelos Base(Base), Modelos com Ajuste de Hiperparâmetros(HP), Modelos com Seleção de Métricas(SM) e Modelos juntando o HP e a $\mathrm{SM}(\mathrm{HP}+\mathrm{SM})$ para os algoritmos de (A) SVM, (B) LR, (C) DT e (D) RF. Os casos estão ordenados de acordo a Tabela $4.1 \ldots \ldots$. . . . . . . . . . 38

4.5 Acurácias de classificação de tarefas cognitivas intradiárias para o SVM depois de aplicar o ajuste de hiperparâmetros e a seleção de métricas. (A) Obtivemos a acurácia da classificação usando a primeira metade dos dados do dia 1 como o conjunto de treinamento e a segunda metade como o conjunto de validação. (B) Obtivemos a acurácia da classificação usando a primeira metade dos dados do dia 2 como o conjunto de treinamento e a segunda metade como o conjunto de validação. . . . . . .

4.6 Acurácias de classificação de tarefas cognitivas interdiárias depois de aplicar o ajuste de hiperparâmetros e a seleção de métricas. Usamos os dados do dia 1 como o conjunto de treinamento e os dados do dia 2 como o conjunto de validação. . . . . . . . . . . . 39

4.7 Classificação de indivíduos. (A) Obtivemos a acurácia da classificação usando a primeira metade dos dados do dia 1 como o conjunto de treinamento e a segunda metade como o conjunto de validação. (B) Obtivemos a acurácia da classificação obtida usando os dados do primeiro dia como o conjunto de treinamento e os dados do segundo dia como o conjunto de validação. . . . . . . . . . . . . . . . . . . . . . . . 40

5.1 Matriz de confusão para identificação dos quatro estados cognitivos desenvolvidos no mesmo dia. . . . . . . . . . . . . . . . . . . . . . . . 43

5.2 Matriz de confusão para identificação dos quatro estados cognitivos desenvolvidos em dias diferentes. . . . . . . . . . . . . . . . . . . . . . . . . . . 43 


\section{Lista de Tabelas}

2.1 Comparação das características dos Parâmetros do Modelo contra os Hiperparâmetros do Modelo . . . . . . . . . . . . . . . . . . . . . . . . . . . . 17

2.2 Desenho Plackett-Burman em 12 execuções para até 11 fatores . . . . . . . . . . . . 19

3.1 Serviços Bluetooth GATT usados pelo Sensor Polar H10 ${ }^{4} \ldots$. . . . . . . . . . . . . 23

3.2 Média e Desvio Padrão da quantidade de registros de dados coletados para cada tarefa cognitiva nas diferentes sessões. . . . . . . . . . . . . . . 25

3.3 Métricas calculadas para cada janela nas diferentes sessões e usadas para a classificação 29

4.1 Casos de análise agrupando as diferentes atividades cognitivas no mesmo dia e em dias diferentes. . . . . . . . . . . . . . . . . . . . . . . . 32

4.2 Especificação do espaço de busca do SVM definido para o ajuste de hiperparâmetros ${ }^{5}$. 33

4.3 Especificação do espaço de busca do DT definido para o ajuste de hiperparâmetros ${ }^{6}$. 34

4.4 Especificação do espaço de busca do RF definido para o ajuste de hiperparâmetros ${ }^{7}$. 34

4.5 Especificação do espaço de busca do LR definido para o ajuste de hiperparâmetros ${ }^{8}$. 34

4.6 Porcentagem de melhoria em todos os casos de análise em relação ao caso base após da aplicação de ajuste de hiperparâmetros em cada algoritmo de ML. A primeira linha mostra o porcentagem de casos melhorados e a segunda o porcentagem de casos sem melhora. . . . . . . . . . . . . . . . . . . . . 35

4.7 Acurácias máximas encontradas após da aplicação do ajuste de hiperparâmetros em cada algoritmo de ML. A primeira linha mostra as acurácias do modelo base, a segunda linha mostra as acurácias aplicando o ajuste de hiperparâmetros e a terceira mostra a taxa de melhoria. . . . . . . . . . . . . . . . . . 36

4.8 Porcentagem de melhoria em todos os casos de análise em relação ao caso base após da aplicação de seleção de métricas em cada algoritmo de ML. A primeira linha mostra o porcentagem de casos melhorados e a segunda o porcentagem de casos sem melhora . . . . . . . . . . . . . . . . . . . . 37

4.9 Acurácias máximas encontradas após da aplicação de seleção de métricas em cada algoritmo de ML. A primeira linha mostra as acurácias do modelo base, a segunda linha mostra as acurácias aplicando a seleção de métricas e a terceira mostra a taxa de melhoria. . . . . . . . . . . . . . . . . . . . . . 37

4.10 Porcentagem de melhoria em todos os casos de análise em relação ao caso base após da aplicação de ajuste de hiperparâmetros com seleção de métricas em cada algoritmo de ML. A primeira linha mostra o porcentagem de casos melhorados e a segunda o porcentagem de casos sem melhora. 
4.11 Acurácias máximas encontradas após da aplicação do ajuste de hiperparâmetros com seleção de métricas em cada algoritmo de ML. A primeira linha mostra as acurácias do modelo base, a segunda linha mostra as acurácias aplicando o ajuste de hiperparâmetros e a terceira mostra a taxa de melhoria. . . . . . . . . . . . . . 38

5.1 Acurácia máxima para cada caso de análise interdiário . . . . . . . . . . . . . . . . 41 


\section{Capítulo 1}

\section{Introdução}

A decodificação do cérebro, ou seja, a discriminação de estados cognitivos específicos, ganhou muita atenção nas últimas décadas $\left[\mathrm{MHN}^{+} 04\right]$. As abordagens padrão de decodificação cerebral têm produzido resultados notáveis em termos de precisão de classificação. Entretanto, elas ainda apresentam algumas limitações críticas [HR06]. A maioria desses estudos precisa de informações explícitas sobre o tempo de eventos cognitivos, ou seja, eles exigem tempos de início e parada precisos para cada estado. Consequentemente, os desenhos dos experimentos se tornam muito artificiais. Eles não reproduzem fenômenos naturais, como o processamento cognitivo contínuo e dirigido pelo sujeito.

Uma alternativa é a conectividade funcional em imagens de ressonância magnética, que examina as correlações de sinais de imagem dependente do nível de oxigênio no sangue (BOLD, do inglês Blood Oxygen Level Dependent Imaging) através de regiões cerebrais. Uma vantagem é que ela pode analisar estados únicos de fluxo livre. Esta abordagem tem sido aplicada mais comumente aos dados de imagens de ressonância magnética funcional (fMRI, do inglês functional Magnetic Resonance Imaging) em estado de repouso para revelar uma série de redes cerebrais, com perfis espaciais e temporais distintos correspondentes a funções canônicas como visão, audição, linguagem, memória de trabalho, atenção visuoespacial, processamento de saliência(importância) no cérebro e memória episódica [BZYHH95, $\mathrm{HPS}^{+} 02, \mathrm{GKRM03}, \mathrm{KKJ}^{+}$03, $\mathrm{BDDS}$ 5, $\mathrm{BPJ}^{+}$06, $\mathrm{DRB}^{+}$06, $\mathrm{SMS}^{+} 07$, $\mathrm{KSR}^{+}$09, $\mathrm{SHY}^{+}$09]

Nesse sentido, $\left[\mathrm{SRR}^{+} 12\right]$ estendeu o alcance da decodificação do estado do cérebro. Eles perguntaram se poderiam usar padrões de conectividade funcional dentro e através das redes cerebrais para distinguir entre quatro estados cognitivos dirigidos pelo sujeito: repouso não dirigido, recuperação de memórias episódicas recentes, canto e subtrações em série. Eles adquiriram a imagem em corridas contínuas de 10 minutos, sem apresentação de estímulo e sem marcos temporais impostos pelo investigador, exceto o início e o final da varredura.

Embora todas essas pesquisas sejam bem-sucedidas, as tecnologias de decodificação cerebral ainda não estão presentes em nossa vida diária [Zeh15]. Uma das principais dificuldades para o desenvolvimento de interfaces de decodificação cerebral úteis para o dia-a-dia é a coleta de dados. Geralmente coletamos sinais cerebrais usando eletroencefalografia (EEG), eletrocorticografia (ECoG), ressonância magnética funcional (fMRI), ou mais recentemente, espectroscopia funcional de infravermelho próximo (fNIRS, do inglês functional Near-Infrared Spectroscopy). Estas tecnologias são muito caras, apresentam baixa razão sinal/ruído e o indivíduo não pode se mover durante a coleta de dados. Finalmente, os métodos baseados no sinal do cérebro ignoram o resto do corpo [Tha13]. A inclusão de todas as informações do corpo é atualmente impossível. Portanto, uma forma natural de avançar nas metodologias de decodificação cerebral seria incluir informações comprimidas de alta qualidade sobre como o corpo interage com o cérebro. Uma maneira de conseguir isso é usar a interocepção, ou seja, a percepção das condições fisiológicas do corpo [Cra03].

Curiosamente, a interocepção pode ser avaliada monitorando a atividade cardíaca [PTB14]. E podemos medir facilmente a frequência cardíaca (FC) por meio da eletrocardiografia (ECG). O ECG não é invasivo, tem uma configuração simples e apresenta uma relação sinal/ruído de magnitude 
mais alta do que as tecnologias de varredura cerebral. Também, o FC representa o estado interno do organismo; ou seja, é um parâmetro global que representa o estado comportamental do corpo, porque é considerado um indicador do Sistema Nervoso Autônomo (ANS, do inglês Autonomous Nervous System) [FFC19]. Por exemplo, as oscilações da FC na frequência de $0,1 \mathrm{~Hz}$ estão associadas a diferentes atividades nervosas simpáticas [Jul06]. Além disso, é bastante evidente que podemos discriminar algumas atividades físicas usando o FC. Por exemplo, a FC é maior ao correr do que ao caminhar. No entanto, a questão não intuitiva é: podemos discriminar estados cognitivos conduzidos pelo sujeito analisando apenas a atividade cardíaca? Aqui, com base nos mesmos experimentos realizados por [SRR ${ }^{+} 12$ ], foi possível demonstrar que podemos discriminar entre estados cognitivos impulsionados pelo sujeito de fluxo livre usando o sinal cardíaco.

\subsection{Considerações Preliminares}

Nos últimos anos, diferentes métricas foram desenvolvidas para os biomarcadores humanos (sinais de origem elétrica, química e acústica que transportam informações ocultas em sua estrutura), especialmente para obter informações sobre o estado de saúde, risco de uma doença ou a esperança de vida de uma pessoa. Além disso, foram feitos esforços dentro do campo da Interação Humano Computador (HCI, do inglês Human Computer Interaction) e da neurociência para desenvolver técnicas que permitam tratar os sinais dos biomarcadores que podem ser controlados pelo cérebro e desta forma criar interfaces que permitam uma comunicação entre o cérebro e o computador. Neste trabalho, focamos em um importante biomarcador, a Variabilidade da Frequência Cardíaca (HRV, do inglês Heart Rate Variability), utilizando técnicas de processamento de sinais e aprendizado de máquina. De acordo com [Alt17], a análise dos biomarcadores com as técnicas mencionadas nos permite:

- Melhorar a precisão das medições dos biomarcadores.

- Ajudar a caracterizar e compreender os sinais e processos fisiológicos.

- Separar o processo fisiológico desejado de outros processos observados.

- Diagnosticar doenças a partir destes sinais que refletem a função de vários órgãos.

- Na terapia automatizada, modificar o comportamento de um processo fisiológico, por exemplo um marca-passo.

\subsection{Objetivos}

O principal objetivo deste trabalho é identificar diferentes estados cognitivos orientados pelo participante do experimento, baseado nas métricas de variabilidade de frequência cardíaca de forma automatizada, com técnicas de processamento de sinais e aprendizagem de maquina.

Assim, nossos objetivos específicos são:

1. Monitorar, anotar e armazenar os dados relativos ao HRV em experimentos controlados.

2. Pré-processar e aplicar filtros aos sinais coletados.

3. Extrair e avaliar as métricas do HRV (métricas no domínio de tempo, no domínio da frequência e métricas não lineares).

4. Modelar e validar o classificador de atividades baseado nas métricas do HRV.

5. Identificar os estados cognitivos.

6. Otimizar os modelos de classificação.

7. Identificar as métricas mais importantes para a classificação nos diferentes casos de análise. 


\subsection{Contribuições}

Este trabalho contribui fornecendo o HRV como uma alternativa para identificar atividades cognitivas orientadas pelo mesmo sujeito abrindo assim caminho a futuros trabalhos no campo da interação humano computador e neurociência.

\section{Artigo submetido}

Os resultados obtidos a partir dos dados coletados de HRV com a otimização dos classificadores com as técnicas de Desenho de Experimento permitiram ter resultados comparáveis a dispositivos mais sofisticados os quais foram compilados no trabalho "Classification of Subject-Driven Cognitive States Based on HeartRate Variability"de Carlos Enrique Paucar Farfán, Grover Castro Guzman, Pedro Bruel ,Alfredo Goldman, Daniel Yasumasa Takahashi, André Fujita.

\section{Banco de Dados}

As métricas do HRV no domínio do tempo, no domínio da frequência e as métricas não lineares dos 25 participantes (Idade Média $=30,2 ;$ Desvio Padrão $=4,9$ ), que foram coletadas em duas sessões diferentes, onde em cada sessão executaram 4 atividades cognitivas.

\subsection{Motivação}

A principal motivação deste projeto foi entender melhor os processos psicofisiológicos compartilhados entre o cérebro e o coração em diferentes estados cognitivos de uma pessoa, utilizando um sensor compacto de baixo custo, afim de viabilizar a criação de soluções computacionais, em áreas como:

- Terapias para insônia.

- Avaliação da Neuropatia Autonômica Diabética.

- Otimização do desempenho esportivo e identificação do sobre-treinamento esportivo.

- Analisar o equilíbrio entre os ramos simpático e parassimpático do sistema nervoso autônomo.

- Prevenção de arritmias, insuficiências cardíacas.

- Estratificação do infarto agudo do miocárdio.

- Detecção de patologias cardíacas.

- Avaliação da hipertensão arterial, fibromialgia, síndrome da fadiga crônica.

- Estratificação do risco de morte súbita cardíaca após um infarto agudo do miocárdio.

- Avaliação da neuropatia diabética.

- Indicador da síndrome metabólica.

- Desempenho atlético.

- Marcador de nível de estresse.

- Entre outros.

\subsection{Organização do Trabalho}

No Capítulo 2 é feita a revisão bibliográfica dos fundamentos básicos do sinal ECG, estados cognitivos, variáveis que influenciam a HRV, o hardware utilizado, a interpretação das métricas da HRV, os algoritmos de aprendizagem de máquina utilizados, o ajuste de hiperparâmetros, as técnicas de seleção de métricas e as técnicas de desenho de experimentos. No Capítulo 3 é apresentada a 
metodologia de coleta de dados e o pre-processamento dos dados até o calculo das métricas da HRV. No capítulo 4 é descrita o modelagem dos classificadores, os casos de análise definidos, a especificação dos espaços de busca para o desenho de experimentos no ajuste de hiperparâmetros (HP), a importância e a seleção de métricas (SM) e a classificação dos participantes. Finalmente, o Capítulo 4 apresenta a Discussão de Resultados e Conclusões. Nos anexos se encontra o endereço web do Banco de Dados coletado e a autorização do comité de ética para coletar dados em humanos. 


\section{Capítulo 2}

\section{Conceitos}

\subsection{Fundamentos básicos do sinal ECG}

O ECG é o registro gráfico da atividade elétrica do coração. Para uma melhor análise e interpretação é necessário entender como é gerado este sinal, começando desde o nível celular, onde a informação é transmitida eletroquimicamente. O nó sinoatrial produz espontaneamente um potencial de ação (PA), o que determina geralmente a frequência cardíaca [Alt17], contraindo e relaxando os átrios e ventrículos, e ao mesmo tempo despolarizando e repolarizando as células.

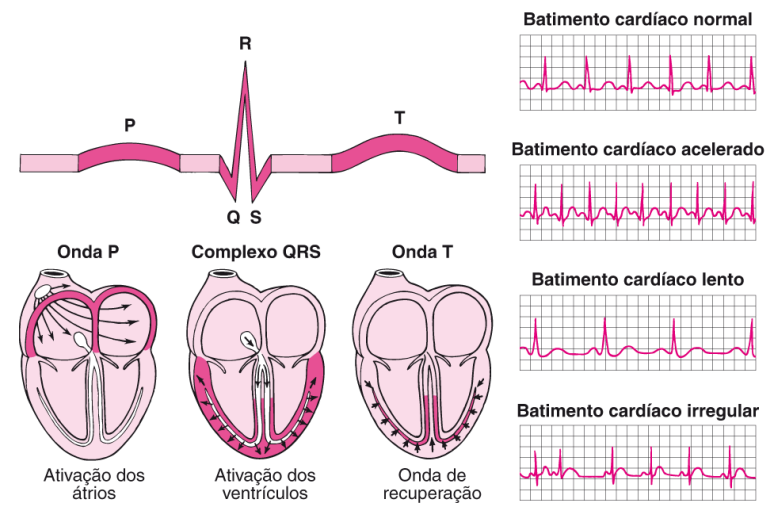

Figura 2.1: Comportamentos das ondas de ECG em estados de ativação(despolarização) e recuperação(repolarização) dos átrios e ventrículos do coração. ${ }^{1}$

A atividade elétrica do coração é causada pelas variações na voltagem dos PAs de milhões de células cardíacas, tal que o ECG reflete a propagação do impulso elétrico através do sistema de condução do coração [SL05]. Como se pode ver na Figura 2.1, o sinal do ECG tem ondas que mostram diferentes estados do coração:

- A onda P reflete a despolarização dos átrios.

- O complexo QRS reflete a despolarização dos ventrículos.

- A onda T reflete a repolarização ventricular.

- A onda $\mathrm{R}$ representa o estímulo elétrico das células que passam pela porção principal das paredes ventriculares. É a onda de maior voltagem, gerado pela quantidade de trabalho que as células tem que fazer por causa do espessor das paredes ventriculares. É por isso que a onda $\mathrm{R}$ é a maior onda gerada durante a condução normal.

Dentre estas ondas, a que tem maior relevância no cálculo das métricas é a onda $\mathrm{R}$, porque a partir desta conseguimos obter o intervalo RR, que é o tempo entre cada pico $\mathrm{R}$ do sinal do ECG, além de ser um indicativo da ocorrência de um batimento cardíaco.

\footnotetext{
${ }^{1}$ https://www.msdmanuals.com/-/media/manual/home/images/cvs_ecg_reading_pt.gif?la $=$ pt\&thn $=0$
} 


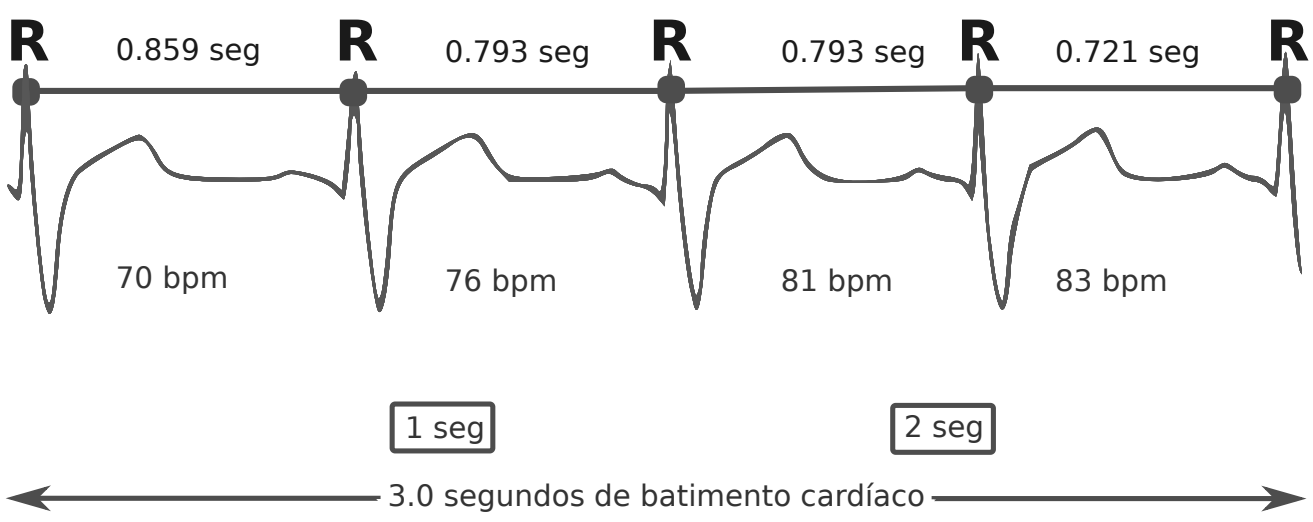

Figura 2.2: Variabilidade no intervalo entre batimentos. O eixo $X$ representa o tempo em segundos e o eixo $Y$ representa a amplitude do sinal em milivolts. ${ }^{2}$

\subsection{Estados cognitivos}

A cognição é a capacidade que temos os humanos para perceber e inferir informação baseado em experiências vivenciadas. Também se pode dizer que é a manifestação do processamento neuronal [BMMFT18]. Diferentes estudos focam em mensurar a capacidade cognitiva no desempenho de diferentes habilidades cognitivas (por exemplo, atenção seletiva, memória, lógica e raciocínio, velocidade de processamento) e os mecanismos subjacentes a essas habilidades (tais como, a herança genética, o ambiente físico, o entorno social e a personalidade) [BMMFT18] os quais produzem as diferenças intra e/ou interindividuais, uma vez que esses mecanismos podem afetar essas habilidades e por sua vez o desenvolvimento cognitivo. Em decorrência existem pesquisas que estudam as consequências da variação cognitiva nos humanos como por exemplo a deterioração cognitiva ao longo do tempo como no envelhecimento [ZAHHD ${ }^{+} 14$ ], em pessoas com ansiedade [EDSC07], em pessoas com depressão [ $\left.\mathrm{MCP}^{+} 10\right]$, com demência, Alzheimer e outras doenças mentais [DC04]. Além disso uma melhor compreensão da cognição humana é importante para entender diferentes doenças tais como obesidade [RMF $\left.{ }^{+} 16\right]$, escleroses múltipla [LTMC08], outros pesquisas estudaram o desempenho cognitivo com os maus hábitos como sua relação com os a privação de sono [APK07].

A medição dos processos cognitivos requer cuidado no desenho experimental e analítico, por tanto é necessário fazer repetições dos experimentos para ter dados mais fidedignos, já que medidas individuais podem acarretar erros [BMMFT18], aumentando assim a confiabilidade da medida da capacidade cognitiva. Diferenças no desempenho cognitivo entre indivíduos foram demonstradas em diversos estudos [Mik97].

A avaliação cognitiva computadorizada tem revolucionada a forma de medir o desempenho cognitivo, além de permitir identificar diferentes funções cognitivas, que são responsáveis pela regulação de diferentes comportamentos específicos. Técnicas modernas de identificação de funções cognitivas como o (CANTAB, do inglês Cambridge Neuropsychological Test Automated Battery [RJO+ 94], permitiram distinguir diferentes funções cognitivas, como são a Cognição Social, a Função Executiva, a Memoria, a Atenção e a Velocidade Psicomotora.

\subsection{Decodificação de atividades cerebrais}

A decodificação de atividades cerebrais é um dos principais objetivos da neurociência cognitiva $\left[\mathrm{RKG}^{+} 17\right]$, com ajuda da automatização computadorizada é possível coletar os dados com medidas precisas e minimizando assim o potencial de erros e preconceitos do administrador do experimento, trazendo assim vantagens em relação aos testes tradicionais. Sobre esta base tecnológica foram desenvolvidas diversas técnicas para encontrar os padrões da atividade cerebral, é onde o aprendizagem de máquina (ML, do inglês Machine Learning) tomou um lugar muito importante

\footnotetext{
${ }^{2}$ https://www.sciencedirect.com/topics/medicine-and-dentistry/interbeat-interval

${ }^{3}$ https://www.cambridgecognition.com/blog/entry/what-is-cognition
} 


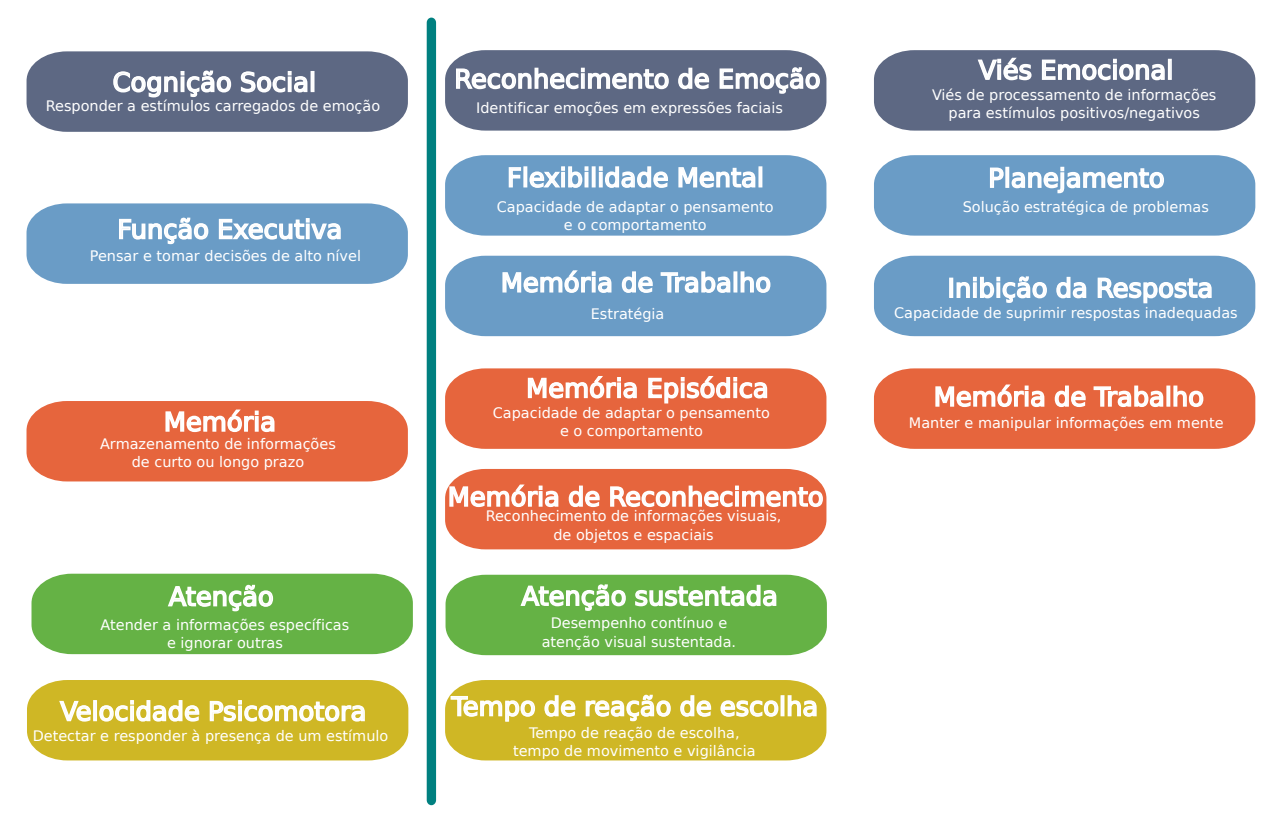

Figura 2.3: Especificidade do dominio da cognição e exemplos de processos cognitivos subjacentes a esses mecanismos. Figura Adaptada ${ }^{3}$.

nesta tarefa, principalmente para encontrar esses padrões de forma automatizada na decodificação cerebral, sobretudo quando se tem grandes bancos de dados com muitas características para analisar. A maioria destes bancos de dados são construídos a partir de determinados estímulos que geralmente são indicados pelo administrador do experimento. $\mathrm{Na}$ atualidade, existem diferentes pesquisas conduzidas a identificar atividades cerebrais, como as que pretendem identificar as fases de sono [ASS ${ }^{+}$16], identificação de emoções [KL16], identificação dos tipos de memória [Slo18], avaliação do desempenho cognitivo [EPB $\left.{ }^{+} 11\right]$, identificação do medo [MF19], avaliação da navegação espacial [GRP00, SRR ${ }^{+}$12], avaliação na toma de decisões [NJHH14], identificação da atenção seletiva [BAJ $\left.{ }^{+} 19\right]$, comparação de processos neuronais entre pessoas [CN14], entre outras pesquisas. A grande maioria destas pesquisas servem de base para construir Interfaces Humano Computador (IHC), tales como interfaces neuronais para funções motoras, funções sensitivas e de estimulação [GQVMCJ07].

Entre as principais aplicações da decodificação da atividade cerebral estão os estudos de IHC, onde principalmente foram explorados abordagens não invasivas como as imagens de ressonância magnética funcional (fMRI) [HR06], magneto-encefalografia (MEG) [CYBB17] e eletroencefalografia (EEG) [KHH14].

Um progresso considerável foi feito nas IHC, baseado em técnicas não invasivas [POS $\left.{ }^{+} 17\right]$, mas é importante destacar que as técnicas invasivas permitem uma decodificação mais precisa $\left[\mathrm{LHW}^{+}\right]$ como no caso dos sinais derivados do eletrocorticograma (ECoG) $\left[\mathrm{PSBA}^{+} 12\right]$ que permitem aos participantes aprender a controlar e modular as peças biomecânicas dentro de poucas sessões, para adquirir um movimento pretendido, em comparação com a necessidade de muitas sessões de treinamento das técnicas não invasivas. A melhora destas técnicas de decodificação neuronal estão possibilitando avanços em IHC, como por exemplo que as peças biomecânicas se adaptem aos sinais cerebrais da pessoa em vez de que a pessoa se adapte às peças.

A maioria destas pesquisas são direcionados para ajudar a pessoas com disfunções neurológicas, como pacientes epilépticos, autistas e pessoas com deficiências biomecânicas que precisem melhorar sua mobilidade ou funções motoras, neste último caso os sinais de controle neural ideais para cada razão particular também estão sendo revelados ou constantemente revisados e atualizados, bem como sendo associados a sinais de outras regiões do cérebro, para ter assim métodos combinados e torná-los disponíveis, com a finalidade de que a decodificação permita prever exatamente o movimento pretendido.

$\left[\mathrm{LHW}^{+}\right]$. 


\subsection{Hardware selecionado}

Para coletar os dados da atividade elétrica do coração são usados comumente dispositivos complexos de alta precisão, que normalmente usam eletrodos que são colocados no tórax e extremidades, além disso precisam de um console que processa e gerencia os sinais recebidos para produzir o sinal do ECG.

Para este trabalho consideramos utilizar uma alternativa mais compacta e econômica como os sensores de fita de eletrodos, que são colocados no tórax. Estes sensores garantem a mesma confiabilidade de dados do sinal ECG dos dispositivos convencionais mais complexos. Por exemplo, o caso do sensor Polar S810i [VSP ${ }^{+}$08] que foi comparado na análise das métricas da HRV no domínio do tempo e da frequência, onde os autores concluíram que o sensor de fita consegue entregar resultados consistentes durante os estados de relaxamento e exercício. Este tipo de sensores, especificamente os da família Polar, como o H7 [GDN16, PSA ${ }^{+}$17, ALAAANAT] e o H10 [SSNM20] mostraram desempenho ótimo em diversos experimentos, como podemos ver na Figura 2.4 onde mostra a comparação entre o sensor Polar H7 e um ECG estacionário.
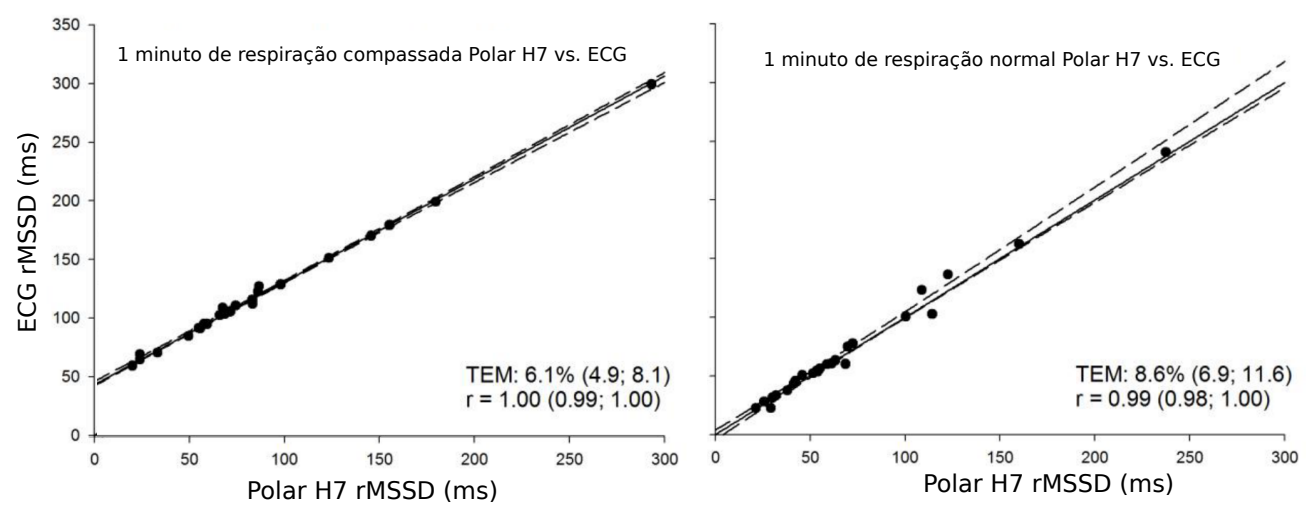

Figura 2.4: Gráficos de correlação do sensor de frequência cardíaca Polar H7 vs ECG durante respiração guiada e normal da métrica $r M S S D$. A linha preta sólida representa a linha de equivalência $(r=1,0)$. Na parte inferior do gráfico esta o Erro Técnico de Medição (TEM, do inglês Technical Error of Measurement) dos 29 participantes avaliados. Adaptado de [PSA+17].

Neste trabalho optamos pelo sensor Polar H10, devido aos antecedentes citados. O bom desempenho do sensor polar $\mathrm{H}_{10}{ }^{4}$ é devido a que este coleta os dados a uma frequência de $1000 \mathrm{~Hz}$ o que o torna tão preciso quanto outros equipamentos mais sofisticados. Os dados são processados no mesmo sensor, tal que entregam a frequência cardíaca e intervalos $\mathrm{RR}$ a uma frequência de $1 \mathrm{~Hz}$.

Entre outras características deste dispositivo é que utiliza o protocolo de comunicação (BLE, do inglês Bluetooh Low Energy), que é uma tecnologia de comunicação entre dispositivos de curto alcance, como telefones celulares e computadores. A tecnologia BLE é um novo padrão de comunicação entre os sensores, é uma melhora ao conhecido protocolo de comunicação Blueetooth, porque permite um consumo de energia baixo entre os dispositivos implicados [DYL19].

\subsection{Variabilidade de Frequência Cardíaca}

Para entender a importância da Variabilidade da Frequência Cardíaca (HRV, do inglês Heart Rate Variability) é necessário saber o que são os intervalos RR e como são calculados. Os intervalos RR são a distância em milissegundos (ms) entre os complexos QRS, especialmente identificando e ressaltando a distância entre picos RR consecutivos, como se pode ver na Figura 2.2. A partir dos intervalos RR se pode inferir o cálculo da Frequência Cardíaca (HR, do inglês Heart Rate), que é o número de batimentos por segundos ou pode-se dizer que é a velocidade que o coração está batendo

\footnotetext{
${ }^{4}$ https://support.polar.com/e_manuals/H10_HR_sensor/Polar_H10_user_manual_English/Content/ Resources/Printed_documentation/manual.pdf
} 
em um determinado momento. As flutuações nos intervalo RR e o HR são inversamente proporcionais, o que indica que ambos mantêm uma relação não linear [BKK $\left.{ }^{+} 17\right]$. Uma representação deste comportamento entre os intervalos RR e a frequência cardíaca é mostrada na Figura 2.5.

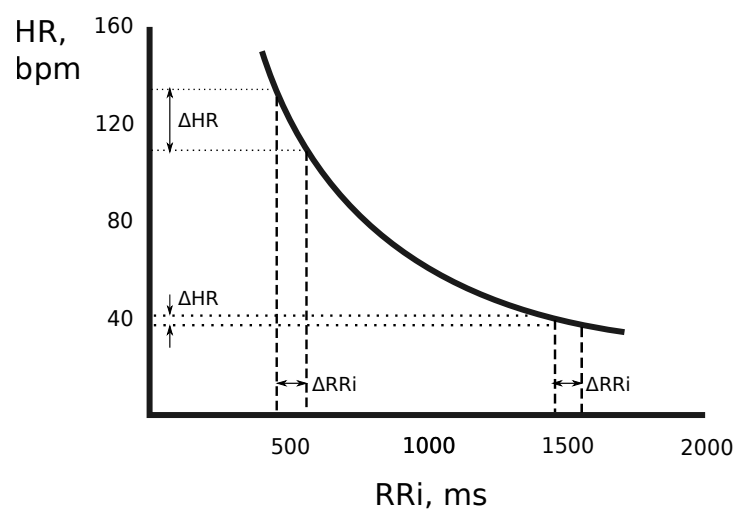

Figura 2.5: A relação entre a frequência cardíaca $(H R)$ e o intervalo $R$ - $R$ (RRi) presenta um comportamento hiperbólico; uma variação no RRi ( $\Delta R R i)$ resulta em mudanças marcadamente diferentes no $H R(\Delta H R)$. Adaptado de [DT16].

O HRV ou também conhecido como variabilidade do comprimento do ciclo cardíaco é um fenômeno fisiológico de variação no intervalo de tempo entre batimentos cardíacos ou também entre os intervalos $\mathrm{RR}\left[\mathrm{MBC}^{+} 96\right]$, tal que é considerado como um biomarcador muito importante do Sistema Nervoso Autônomo (ANS, do inglês Autonomous Nervous System) [MBC ${ }^{+}$96], que tem um rol importante na identificação de diversas patologias, por exemplo um baixo HRV foi demostrado em ser um forte preditor de mortalidade por arritmia cardíaca [Szt04], proporcionando assim para os cardiologistas um indicador de estudos clínicos. O HRV é melhor descrito pelas suas métricas, onde para o desenvolvimento deste projeto decidimos trabalhar com as seguintes:

- Métricas no domínio de tempo.

- Métricas no domínio da frequência.

- Métricas não lineares.

Mediante o toolbox gHRV $\left[\mathrm{RLLV}^{+} 14\right]$ que tem funções implementadas foi possível calcular as mencionadas métricas.

\subsubsection{Métricas no domínio do tempo}

Estas métricas são calculadas tradicionalmente a partir da série de intervalos NN, que são os intervalos RR sem valores ectópicos(ruídos). Algumas destas métricas entregam uma melhor informação de acordo ao tempo coletado que pode ser de longo (até 24 horas) ou de curto (até 5 min) prazo, a fim de representar as informações relacionadas principalmente à atividade parassimpática ou simpática $\left[\mathrm{BKK}^{+} 17\right]$. Nas seguintes fórmulas, a terminologia usada estava dada por $\mathrm{RR}_{i}$ que é o i-ésimo intervalo $\mathrm{RR}, \mathrm{HR}_{i}$ que é a i-ésima frequência cardíaca e $n$ que é o tamanho da janela do intervalo de dados considerado para o cálculo das seguintes métricas:

- Média dos intervalos RR (MRR): Indica como o HRV esteve durante um intervalo de tempo. Quanto maior for esta medida, maior é o HRV e vice-versa [SG17]. Geralmente os valores variam entre $600<\mathrm{MRR}<1200$. Unidade em milissegundos $(\mathrm{ms})$.

$$
\mathrm{MRR}=\frac{1}{n} \sum_{i=1}^{n} \mathrm{RR}_{i}
$$


- Frequência cardíaca média (MHR): Normalmente uma mhr baixa indica repouso, enquanto uma mhr alta corresponde algum esforço gerado. Geralmente em estado de repouso os valores variam entre $60<\mathrm{MHR}<100$. Unidade em batimentos por minuto $(\mathrm{bpm})$.

$$
\mathrm{MHR}=\frac{1}{n} \sum_{i=1}^{n} \mathrm{HR}_{i}
$$

- Desvio padrão dos intervalos NN (SDNN): Reflete a variação global das séries RR, sua utilização é recomendável em registros de longo prazo. Ajuda na estratificação médica de risco cardíaco e predição tanto da morbidade quanto da mortalidade $\left[\mathrm{MBC}^{+} 96\right]$. Geralmente em estado de repouso os valores variam entre $20<$ SDNN $<60$. Unidade em milissegundos $(\mathrm{ms})$.

$$
\mathrm{SDNN}=\sqrt{\frac{1}{n-1} \sum_{i=1}^{n}\left(\mathrm{NN}_{i}-\mathrm{MRR}\right)^{2}}
$$

- Coeficiente de variação $\left(\mathbf{C V} \_\mathbf{R R}\right)$ : Expressa a variação entre duas ondas $\mathrm{R}$ consecutivas. A diminuição desta métrica está associada ao envelhecimento. Geralmente em estado de repouso os valores variam entre $4 \%<\mathrm{CV} \_\mathrm{RR}<15 \%$.

$$
\mathrm{CV}_{-} \mathrm{RR}=\mathrm{SDNN} / \mathrm{MRR}
$$

- Raiz quadrada média de diferenças sucessivas de intervalos NN (rMSSD): Útil para estudar a variabilidade a curto prazo. Acentua o conteúdo de alta frequência da série de intervalos NN. É considerada a medida mais relevante e precisa da atividade do Sistema Nervoso Autônomo no curto prazo. Geralmente em estado de repouso os valores variam entre $10<\mathrm{rMSSD}<50$. Unidade em milissegundos $(\mathrm{ms})$.

$$
\mathrm{rMSSD}=\sqrt{\frac{1}{n} \sum_{i=1}^{n-1}\left(\mathrm{RR}_{i+1}-\mathrm{RR}_{i}\right)^{2}}
$$

- Desvio padrão da frequência cardíaca (HRSTD): Reflete a variação global da Frequência Cardíaca, bem como a curto e longo prazos. Unidade em batimentos por minuto (bpm).

$$
\operatorname{HRSTD}=\sqrt{\frac{1}{n-1} \sum_{i=1}^{n-1}\left(\mathrm{HR}_{i}-\mathrm{MHR}\right)^{2}}
$$

- Porcentagem de NN50 (pNN50): Quociente de número de pares de intervalos NN sucessivos que diferem em mais de 50ms (NN50) entre número total de intervalos NN. Útil para estudar a variabilidade de curto prazo. Geralmente em estado de repouso os valores variam entre $4<$ pNN50 $<30$.

$$
\mathrm{pNN} 50=\frac{\#(\mathrm{NN}>50)}{\#(\mathrm{NN})}
$$

As métricas mencionadas têm importância clínica, tal que algumas pesquisas [RPR ${ }^{+}, \mathrm{SMWY} 13$, $\mathrm{CKK}^{+}$17] indicam que uma baixa HRV (ou menor variabilidade entre batimentos cardíacos) significa que o corpo está sob estresse devido a treinamento físico excessivo, emoções intensas por causa de estressores que podem ser internos ou externos. Uma maior HRV (ou maior variabilidade entre os batimentos cardíacos) geralmente significa que o corpo tem uma grande capacidade de tolerar o estresse ou está se recuperando facilmente do estresse acumulado. Quando a pessoa está em repouso, uma alta HRV é geralmente favorável e uma baixa HRV é desfavorável. Quando a pessoa está em 
um estado ativo, uma HRV mais baixa é geralmente favorável, enquanto uma HRV alta pode ser desfavorável [Alt17]. Portanto é recomendado medir estes valores em estados de repouso e atividade para ter um melhor panorama do estado de saúde da pessoa.

\subsubsection{Métricas no domínio da frequência}

A análise espectral nos permite observar e quantificar componentes periódicos incorporados no ritmo cardíaco como picos do espectro de potência. Em sujeitos saudáveis e em repouso, a HRV apresenta um comportamento oscilatório influenciado pela atividade respiratória, pressão arterial e mecanismos de feedback para a regulação da temperatura [Alt17]. Para calcular estas métricas é possível usar a Transformada Rápida de Fourier (FFT) ou modelo Auto-Regressivo (AR). A distribuição da potência absoluta ou relativa são estimadas em quatro bandas (definidas pelo grupo de trabalho da Sociedade Europeia de Cardiologia e da Sociedade Americana de Estimulação e Eletrofisiologia [SG17, $\left.\left.\mathrm{MBC}^{+} 96\right]\right)$ :

- Banda ULF: Potência do espectro na faixa $\leq 0.003 \mathrm{~Hz}$. Corresponde à frequências ultra baixas do espectro de potência da HRV. É recomendável medir usando registros de 24 horas para que possa ter mais importância. [KSB05]. Unidade em milissegundos ao quadrado $\left(m s^{2}\right)$.

- Banda VLF: Potência do espectro na faixa 0.0033-0.04Hz. Corresponde à frequências muito baixas do espectro de potência da HRV. Diferentes pesquisas associaram uma baixa potência da VLF à morte arrítmica $\left[\mathrm{BFS}^{+}\right.$92], desordem de estresse pós-traumático, alta inflamação [LBS $\left.{ }^{+} 08, \mathrm{CFS}^{+} 07\right]$ e baixos níveis de testosterona [TLJBE07]. Unidade em milissegundos ao quadrado $\left(m s^{2}\right)$.

- Banda LF: Potência do espectro na faixa $0.04-0.15 \mathrm{~Hz}$. Corresponde à frequências baixas do espectro de potência da HRV. Reflete principalmente a atividade barorreceptora durante as condições de repouso [USMA98]. A potência do LF pode ser produzida tanto pelo Sistema Nervoso Parassimpático (SNP) quanto pelo Sistema Nervoso Simpático (SNS). Unidade em milissegundos ao quadrado $\left(m s^{2}\right)$.

- Banda HF: Potência do espectro na faixa $0.15-0.40 \mathrm{~Hz}$. Corresponde à frequências altas do espectro de potência da HRV. Reflete a atividade parassimpática e é chamada de banda respiratória, pois corresponde às variações da FC relacionadas ao ciclo respiratório [GT07], que é usada frequentemente como indicador do tom vagal cardíaco que a sua vez foi indicado como um indicador fisiológico de estresse [Por95]. Unidade em milissegundos ao quadrado $\left(m s^{2}\right)$.

- LF/HF ratio: Esta métrica pode estimar a relação entre a atividade do sistema nervoso simpático (SNS) e do sistema nervoso parassimpático (SNP) sob condições controladas [SG17]. É a razão entre os valores de LF e HF.

- Potência total: É a soma da energia nas bandas ULF, VLF, LF e HF. Durante a ativação simpática a taquicardia resultante é geralmente acompanhada por uma redução acentuada na potência total, enquanto o inverso ocorre durante a ativação vagal [Mal96]. Unidade em milissegundos ao quadrado $\left(m s^{2}\right)$.

\subsubsection{Métricas não lineares}

A HRV é influenciada por muitas variáveis que têm um comportamento não linear e não oscilatório, como as causadas por arritmias ou movimentos repentinos do corpo, o que torna a análise da HRV mais difícil com as métricas anteriormente descritas. A não linearidade significa que uma relação entre variáveis não pode ser plotada como uma linha reta [Alt17]. Como mencionam alguns estudos [SG17], as medições não lineares indexam a imprevisibilidade de uma série temporal, que 
resulta da complexidade dos mecanismos que regulam a HRV, como por exemplo estresse ou estado de ânimo.

As métricas selecionadas para nossa análise foram:

- Entropia Aproximada (ApEn): Quantifica a imprevisibilidade das flutuações nas series temporais, reflete a verosimilhança que observações de padrões não sejam seguidos por observações similares. Um valor alto de ApEn indica que a série temporal é menos previsível e vice-versa.

$$
A p E n=\ln \left[\frac{C_{m}(r)}{C_{m+1}(r)}\right]
$$

onde $C_{m}(r)$ é a prevalência dos valores repetitivos de tamanho $m$ em series de tempo de frequência cardíaca e $r$ é o critério de similitude[PG94].

- Dimensão Fractal (FracDim): Mede a complexidade do sinal de HRV. Em especial, mostra as características de auto-similaridade nas séries temporais de HRV. O cálculo foi realizado de acordo com o algoritmo descrito em [KFP91].

$$
\text { FracDim }=\frac{\ln \left(C_{m}\left(r_{b}\right)\right)-\ln \left(C_{m}\left(r_{a}\right)\right)}{\ln \left(r_{b}\right)-\ln \left(r_{a}\right)}
$$

onde $m$ também é conhecido como a dimensão de incorporação e $r$ é a longitude de escala.

\subsection{Variáveis que influenciam ao HRV}

Existem muitos fatores que podem influenciar as métricas da Variabilidade de Frequência Cardíaca (HRV), por ser um indicador que mede o equilíbrio do Sistema Autônomo Simpático e o Sistema Autônomo Parassimpático, e estes dependem das seguintes variáveis controláveis (que dependem de nós) e não controláveis (que não dependem de nós), segundo [QH14]:

- O nível de atividade física $\left[\mathrm{BSM}^{+} 07\right]$.

- A ingestão de alimentos foi demostrada que reduz a atividade parassimpática [LZOC].

- A temperatura do ar que afeta a temperatura corporal $\left[\mathrm{MMJ}^{+} 11\right]$.

- A posição do corpo (ex. de pé ou deitado) [OOC86].

- Variáveis psicológicas (ex. emoções, estresse, ansiedade) [CKK $\left.{ }^{+} 17\right]$.

- A idade [OOC86].

- Hora do dia $\left[\mathrm{MMW}^{+} 00\right]$.

- Os alimentos ingeridos (ex. cafeína) [SvMKK02].

- Os medicamentos ingeridos (ex. antidepressivos) $\left[\mathrm{KQG}^{+} 10\right]$.

\subsection{Classificadores de Aprendizagem de Máquina}

\subsubsection{Máquinas de Vetores de Suporte}

O potencial do algoritmo das máquinas de vetores de Suporte (SVM, do inglês Support Vector Machine) para classificar os sinais de frequência cardíaca foi demonstrado em vários trabalhos relacionados sobre a classificação do sinal de HRV [KMN09, ALAAANAT16, PTU14, ASM08]. Os classificadores SVM são baseados em avanços na teoria de aprendizagem estatística. O principal objetivo da classificação SVM é conceber uma maneira computacionalmente eficiente de aprender bons hiperplanos separadores em um espaço de recursos de alta dimensão. Por bons hiperplanos 
queremos dizer aqueles que otimizam o limites de generalização capazes de lidar com amostras de tamanho considerável [CST00]. Como os dados nem sempre podem ser separados linearmente em seu espaço de recursos original, é necessário mapear esses dados com funções de mapeamento $K$ para outros espaços de dimensões altas para encontrar hiperplanos que permitem que as amostras sejam linearmente separáveis maximizando as margens de separação entre as amostras. O principal problema que tenta resolver o SVM é maximizar a separação dos vetores de suporte paralelos ao hiperplano que separa as classes, a largura entre os vetores de suporte dos elementos das diferentes classes, mas isso nos leva a um problema de minimização que finalmente é refletido na equação Lagrangiana $L$ que tem que ser minimizada sujeito a restrições, desta forma se tem a Equação 2.1 que é o Lagrangiano $L$ reduzido, para que a maximização dependa do produto escalar dos pares de vetores de suporte.

$$
L=\sum_{i=1} \alpha_{i}-\frac{1}{2} \sum \sum \alpha_{i} \alpha_{j} y_{i} y_{j} \phi\left(x_{i}\right) . \phi\left(x_{j}\right)
$$

Além disso, a regra de decisão 2.2, que permite classificar as amostras, também dependerá do produto escalar entre as funções de mapeamento das diferentes amostras, .

$$
\sum_{i=1}^{N}\left(\alpha_{i} y_{i} \phi\left(x_{i}\right) \cdot \phi\left(x_{j}\right)+b \geq 0\right)
$$

Para facilitar o cálculo de localização da função de mapeamento, foi definida a função Kernel, que define um produto ponto no espaço de atributos transformado.

$$
K\left(x_{i}, x_{j}\right)=\phi\left(x_{i}\right) . \phi\left(x_{j}\right)
$$

Finalmente a classificação de um vetor $x_{j}$ fora do conjunto de treinamento é realizada por

$$
f\left(x_{j}\right)=\operatorname{sign}\left(\sum_{i=1}^{N}\left(\alpha_{i} y_{i} K\left(x_{i}, x_{j}\right)+b\right)\right)
$$

onde o vetor de treinamento correspondente $x_{i}$ é chamado de vetor de suporte.

Uma das principais características do SVM é o Kernel Trick, aplicável nos casos em que, por exemplo, os dados que não são linearmente separáveis no espaço gerado pelos atributos de entrada podem ser linearmente separáveis no espaço dos atributos obtidos pela aplicação de um determinado Kernel [NC00]. Existem diferentes funções do Kernel que podem ser usadas:

- Kernel Linear: $K\left(x_{i}, x_{j}\right)=x_{i} \cdot x_{j}$

- Kernel Sigmoid : $K\left(x_{i}, x_{j}\right)=\tanh \left(a x_{i} \cdot x_{j}+b\right)$ onde $a$ e $b$ são termos constantes.

- Kernel Polynomial: $K\left(x_{i}, x_{j}\right)=\left(a+x_{i} x_{j}\right)^{b}$ onde $a$ é um termo constante e $b$ é o grau do kernel.

- Kernel de Funções de Base Radial (RBF): $K\left(x_{i}, x_{j}\right)=\exp \left(-a\left\|x_{i}-x_{j}\right\|^{2}\right)$ onde $\left\|x_{i}-x_{j}\right\|$ é a distancia Euclidiana entre $x_{i}$ e $x_{j}$.

Implementações posteriores permitiram que o SVM fosse estendido para problemas de classificação multiclasses com diversas técnicas [HL02]. Neste estudo, diferentes funções de kernel com seus respectivos parâmetros foram avaliadas para o procedimento de ajuste de hiperparâmetros, que detalhamos na Tabela 4.2 .

\subsubsection{Regressão Logística}

A regressão logística (LR, do inglês Logistic Regression) é um modelo estatístico ou algoritmo que tem a capacidade de resolver problemas de classificação. O LR calcula as mudanças no logaritmo das 
probabilidades da variável de resposta, ao invés de mudanças na própria variável de resposta, como a regressão linear faz. Como o logaritmo das probabilidades está linearmente relacionado às variáveis explicativas, a relação regressiva entre a resposta e as variáveis explicativas não é linear [SE05].

Baseado no número de categorias a regressão logística pode ser organizada em:

Binomial: A variável resposta pode ter só dois possíveis valores, 0 ou 1 que podem ter distintos significados, em sua forma básica usa uma função logística ou também conhecida como função sigmóide para modelar uma variável dependente binária através de um conjunto de dados explicativos [DOM02].

$$
\text { Função sigmóide }=\sigma(x)=f(x)=\frac{1}{1+e^{-x}}
$$

A representação usada para a regressão logística binomial é:

$$
y=e^{\left(b_{0}+b_{1} x\right)} /\left(1+e^{\left(b_{0}+b_{1} x\right)}\right)
$$

Multinomial: A variável objetivo pode ter 3 ou mais possíveis valores. É uma extensão da LR binaria para mais de duas classes, que usa a função softmax, esta função comprime todos os valores no intervalo $[0,1]$ e a soma dos elementos é 1.

$$
\operatorname{softmax}(x)_{i}=\frac{e^{x_{j}}}{\sum_{j=1}^{n} e^{x_{j}}}
$$

Existem dois métodos comuns para realizar a classificação multi-classe usando o algoritmo de regressão logística de classificação binária: um contra de todos e um contra um, dado $C$ o número de classes, temos que para o caso um contra todos, treinamos o classificador binário separando para cada classe as outras $C-1$ classes e executamos todas essas classificações obtendo assim um score em cada combinação. Para o outro caso um contra um, treinamos o classificador escolhendo cada 2 classes com um total de combinações $(C-1) / 2$ e escolhemos a classe com votos máximos. Os hiperparâmetros analisados para o algoritmo LR podem ser encontrados na Tabela 4.5.

\subsection{3 Árvores de Decisão}

O algoritmo da árvore de decisão (DT, do inglês Decision Tree) tem uma estrutura de árvore que modela os resultados prováveis por meio de seus ramos que representam as regras de decisão onde cada nó representa um atributo [SH77]. Este algoritmo usa a técnica de "dividir e conquistar" para dividir os registros, transformando o recurso selecionado em um nó de decisão e então este processo é repetido recursivamente em cada subárvore até que uma das seguintes condições coincida.

- Todos os registros correspondem ao mesmo valor da característica.

- Não há mais nós na árvore.

Entre os hiper-parâmetros mais usuais do arvore de decisão estão:

- O critério de impuridade é a função que mede a qualidade de uma partição, geralmente se usam o critério de impureza de Gini e o critério de entropia para a ganancia de informação.

$$
\begin{gathered}
\text { Entropia }=-\sum_{i=1}^{n} p_{i} \log \left(p_{i}\right) \\
\text { Indice Gini }=1-\sum_{i=1}^{n} p_{i}^{2}
\end{gathered}
$$

- A quantidade de atributos necessárias para obter a melhor partição, em diferentes ferramentas de software assume diferentes valores derivados do número de atributos que podem ser a raiz quadrada ou o logaritmo destes. 
- O número mínimo de amostras necessárias para estar em um nó folha.

- O número mínimo de amostras necessárias para particionar um nó interno.

- A profundidade da árvore.

Todas essas características do DT são considerados como hiperparâmetros os quais são avaliados na Tabela 4.3 para o ajuste dos mesmos.

\subsubsection{Florestas Aleatórias}

A (RF, do inglês Random Forest) consiste em um grande número de árvores de decisão individuais que operam como um conjunto, cada árvore individual na floresta aleatória fornece uma previsão de classe e a classe com mais votos torna-se o modelo de previsão [Bre99]. O grande número de modelos (árvores) relativamente não correlacionados operando como um comitê superará qualquer um dos modelos constituintes individuais. Pode-se dizer que se trata de um algoritmo democrático, onde a baixa correlação entre os modelos é a chave, de forma que eles podem produzir previsões por conjunto mais precisas do que qualquer uma das previsões individuais. A razão para esse efeito maravilhoso é que as árvores protegem umas às outras de seus erros individuais. Enquanto algumas árvores podem estar erradas, muitas outras estarão corretas, desta forma esses grupos de árvores são capazes de se mover na direção correta [MS16]. Um termo importante de mencionar é o Ganho de Informação que é uma medida útil para construir as árvores de decisão que é calculado como a diminuição da entropia depois que o conjunto de dados é particionado sobre um atributo.

$$
\text { Ganho de Informação }=\operatorname{Entropia}(T)-\operatorname{Entropia}(T, X)
$$

onde $T$ é a variável objetivo, $X$ é o atributo onde vai se particionar e Entropia $(T, X)$ é a entropia calculada depois de que os dados foram divididos no atributo $X$.

Para o RF são consideradas os mesmos hyperparámetros do que o DT, só que aumentando o número de estimadores ( $n_{-}$estimators) $\left[\mathrm{PFMGO}^{+} 11\right]$ que é a quantidade de árvores na floresta.

Os hiperparâmetros analisados para o algoritmo de RF podem ser encontrados na Tabela 4.4.

\subsection{Métricas de desempenho do classificador}

Matriz de confusão: É uma matriz bidimensional, indexada em uma dimensão pela verdadeira classe de um objeto e na outra pela classe que o classificador atribui, para resumir o desempenho de classificação de um classificador com relação a alguns dados de teste [Pra17].

- Verdadeiros Positivos (VP): São os casos quando a classe atual do punto de dados foi verdadeira e a predição também foi verdadeira.

- Falsos Positivos (FP): São os casos quando a classe atual do punto de dados foi falsa e a predição foi verdadeira. (Erro tipo I)

- Falso Negativo (FN): São os casos quando a classe atual do punto de dados foi verdadeira e a predição foi falsa. (Erro tipo II)

- Verdadeiro Negativo (VN): São os casos quando a classe atual do punto de dados foi falsa e a predição foi falsa.

Métricas derivadas da matriz de confusão: Da matriz de confusão é possível conseguir as seguintes métricas de classificação $\left[\mathrm{SFC}^{+} 11\right]$ :

- Precisão: Indica dentre todas as classificações positivas quantas foram realmente corretas.

$$
\text { Precisão }=\frac{V P}{V P+F P}
$$




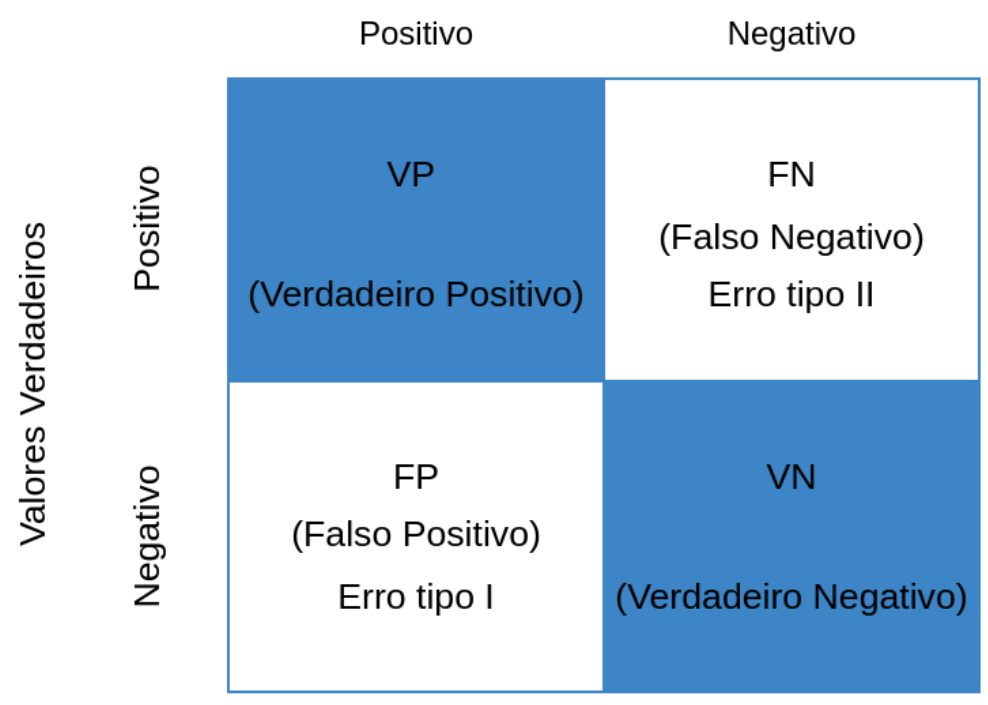

Valores Preditos

Figura 2.6: Matriz de confusão.

- Sensibilidade: Indica dentre todas as situações de classe positivo como valor esperado, quantas estão corretas. Também conhecida como Taxa Positiva Verdadeira (TPV).

$$
T P V=\frac{V P}{V P+F N}
$$

- Especificidade: Indica dentre todas as situações de classe negativa como valor esperado, quantas estão corretas. Também conhecida como Taxa Negativa Verdadeira $(T N V)$.

$$
T N V=\frac{V N}{V N+F P}
$$

- F1-Score: Média harmônica entre Recall e Precisão.

$$
F 1-\text { Score }=2 * \frac{\text { Precisão } * \text { Recall }}{\text { Precisão }+ \text { Recall }}
$$

- Acurácia: É a razão entre o número de predições corretas e o número total de amostras de entrada.

$$
\text { Acurácia }=\frac{V P+V N}{V P+F P+F N+V N}=\frac{\text { Número de predições corretas }}{\text { Número total de predições }}
$$

É recomendável usar quando as classes tem quantidades semelhantes de observações.

\subsection{Otimização de hiperparâmetros}

Em aprendizagem de máquina um hiperparâmetro é um parâmetro cujo valor é usado para controlar o processo de aprendizagem. Em contraste, os valores dos parâmetros do modelo são aprendidos ou inferidos durante o ajuste do modelo de aprendizado sobre o conjunto de treinamento porque se referem à tarefa de seleção de modelo. Um exemplo de um modelo de hiperparâmetro é a quantidade de estimadores que pode usar a Floresta Aleatória (RF), o tipo de Kernel que vai usar o SVM. Dados esses hiperparâmetros, o algoritmo de treinamento aprende os parâmetros dos dados. Na Tabela 2.1 podemos observar as diferenças entre parâmetros e hiperparâmetros. 
Tabela 2.1: Comparação das características dos Parâmetros do Modelo contra os Hiperparâmetros do Modelo

\begin{tabular}{l|l}
\hline \multicolumn{1}{c|}{ Parâmetro do modelo } & \multicolumn{1}{c}{ Hiperparâmetro do modelo } \\
\hline Eles são exigidos pelo modelo ao fazer previsões. & $\begin{array}{l}\text { Eles são freqüentemente usados em processos } \\
\text { para ajudar a estimar os parâmetros do modelo. }\end{array}$ \\
\hline Eles são estimados ou aprendidos com os dados. & $\begin{array}{l}\text { Muitas vezes, eles podem ser definidos } \\
\text { usando heurísticas. }\end{array}$ \\
\hline $\begin{array}{l}\text { Freqüentemente, eles não são definidos } \\
\text { manualmente pelo profissional. }\end{array}$ & $\begin{array}{l}\text { Freqüentemente, eles são especificados } \\
\text { pelo profissional. }\end{array}$ \\
\hline $\begin{array}{l}\text { Eles geralmente são salvos como parte do } \\
\text { modelo aprendido. }\end{array}$ & $\begin{array}{l}\text { Eles geralmente são ajustados para um } \\
\text { determinado problema de modelagem preditiva. }\end{array}$ \\
\hline
\end{tabular}

Nas aplicações de aprendizado de máquina, selecionar os hiperparâmetros apropriados para modelos de classificação é uma etapa crucial que impacta a generalização e o desempenho do classificador $\left[\mathrm{LLY}^{+} 06\right]$, também problemas de reprodutibilidade são esperados quando um grande espaço de busca de hiperparâmetro é apresentado, especialmente quando a pesquisa é feita manualmente [FPea11], para a qual é necessário automatizar esta etapa do modelagem, que é importante no contexto do AutoML (Automated Machine Learning) [BBBK11, BCC12]. A automação de ajuste de hiperparâmetros facilita a comparação de diferentes modelos que recebem o mesmo nível de ajuste para o problema em questão, permitindo uma comparação mais justa desses modelos [FH19]. Além disso, ajuda a melhorar o desempenho de algoritmos de aprendizado de máquina e em diversos estudos permitiu novos desempenhos de ponta [CDM15]. Entre as técnicas mais comuns de otimização de hiperparâmetros estão o Grid Search, Random Search, Bayesian Optimization, Gradient-based Optimization, Evolutionary Optimization, Population-based, Early Stopping-based [FH19].

\subsection{Importância das características}

Reconhecer as características, atributos ou métricas mais importantes das classes permite reduzir o ruido no modelo treinado e assim evitar acurácias baixas, redução de tempo no treinamento e eficiência na administração de recursos computacionais. Por tanto a seleção de características é considerado importante em diversas aplicações de aprendizagem de máquina [Sha15]. Entre os principais métodos que existem para a seleção de atributos estão:

- Métodos de Filtro: São independentes dos algoritmos de ML, por exemplo podemos usar a correlação de Pearson quando a variável de resposta e as características são continuas, mas quando as características são categóricos o ANOVA é recomendável. Para o caso quando a variável de resposta seja categórica é possível usar o Análise Discriminante Linear se os atributos são contínuos e o chi-quadrado, se os atributos são categóricos [SIL07].

- Métodos de Envoltura: Precisam de um algoritmo de ML e usam o desempenho como critério de avaliação, procurando as características mais apropriados para o modelo adicionando ou removendo-os. Entre os métodos mais comuns estão a Seleção para frente (Forward Selection), Eliminação para trás (Backward Elimination), Eliminação recursiva de atributo (Recursive Feature Elimination) [Sha15].

- Métodos Integrados: É implementado sobre algoritmos que tem seus próprios métodos de seleção de características, onde os métodos de filtro e envoltura são combinados. Por exemplo a regressão Lasso e Ridge tem funções de penalidade integradas para reduzir o sobreajuste [Sha15]. 


\subsection{Desenho de Experimentos}

O (DOE, do inglês Experimental Design ou Design of Experiments), é definido como um ramo da estatística aplicada que trata do planejamento, condução, análise e interpretação de testes controlados para avaliar os fatores que controlam o valor de um parâmetro ou grupo de parâmetros [20007]. O DOE é um método sistemático para determinar a relação entre os fatores que afetam um processo e a saída desse processo. Em outras palavras, é usado para encontrar relações de causa e efeito [WA14]. Um dos principais objetivos é obter a máxima informação com o mínimo de experimentos.

Os termos mais usuais na literatura do DOE são:

- Variável Resposta: O resultado do experimento.

- Fator: Variáveis que afetam a variável de resposta e tem varias alternativas.

- Nível: Os valores que um fator pode assumir.

- Fatores Primários: Os fatores cujos efeitos precisam ser quantificados.

- Fatores Secundários: Fatores que impactam o desempenho, mas cujo impacto não estamos interessados em quantificar.

- Replicação: Repetição de todos ou alguns experimentos.

- Unidade Experimental: Entidade que é usada para o experimento.

- Interação: Dois fatores A e B interagem se o efeito de um depende do nível do outro.

O DOE apresenta diferentes tipos de técnicas as quais devem ser usadas segundo o problema, entre as principais estão as seguintes:

\subsubsection{Desenhos Fatoriais Completos}

Usa todas as possíveis combinações em todos os níveis de todos os fatores. Dado $k$ fatores e o i-ésimo fator tendo $n_{i}$ níveis [Mon92]. O número requerido de experimentos é:

$$
n=\prod_{i=1}^{k} n_{i}
$$

\subsubsection{Desenhos Fatoriais Fracionários}

Útil quando o desenho Fatorial Completo resulta num alto número de experimentos, tal que não todos os experimentos poderiam ser executados. Usa subconjuntos de níveis de fatores e as possíveis combinações destes. Dado $k$ fatores e o i-ésimo tendo $n_{i}$ níveis, e subconjuntos de níveis seleccionados $m_{i} \leq n_{i}$ [Mon92] O número requerido de experimentos é:

$$
n=\prod_{i=1}^{k} m_{i}
$$

\subsubsection{Desenhos Aleatórios}

Como o nome indica, este tipo de desenho de experimentos envolve a atribuição aleatória de condições experimentais. No entanto, os números não devem ser escolhidos sem qualquer critério. Este tipo de projeto experimental é surpreendentemente poderoso e frequentemente resulta em uma alta probabilidade de criar um projeto quase ótimo [Woo07]. As etapas simplificadas para o desenho aleatório incluem o seguinte: 
- Escolha uma série de experimentos para executar, não entanto pode ser difícil escolher um número porque depende da quantidade de recuperação de sinal que você deseja.

- Atribua a cada variável um estado com base em uma amostra uniforme. Por exemplo, se houver 5 estados, cada estado tem uma probabilidade de $20 \%$.

Desenhos aleatórios geralmente funcionam bem para grandes sistemas com muitas variáveis, 50 ou mais. Deve haver poucas interações entre as variáveis e muito poucas variáveis que contribuem significativamente.

\subsubsection{Desenho Plackett Burman}

Os desenhos Plackett Burman (PB) são muito úteis para detectar economicamente grandes efeitos principais, assumindo que todas as interações são desprezíveis quando comparadas com os poucos efeitos principais importantes. Da mesma forma que para o Screening Design o Desenho PB ajuda a identificar os efeitos mais significativos quando se tem muitos fatores para considerar [Zey13]. Por exemplo na Tabela 2.2 podemos ver um desenho PB em 12 execuções que pode ser usado para um experimento contendo até 11 fatores.

Tabela 2.2: Desenho Plackett-Burman em 12 execuções para até 11 fatores

\begin{tabular}{|r|r|r|r|r|r|r|r|r|r|r|r|}
\hline & $\boldsymbol{X} \mathbf{1}$ & $\boldsymbol{X} 2$ & $\boldsymbol{X} 3$ & $\boldsymbol{X} 4$ & $\boldsymbol{X} 5$ & $\boldsymbol{X} 6$ & $\boldsymbol{X} \mathbf{7}$ & $\boldsymbol{X} 8$ & $\boldsymbol{X 9}$ & $\boldsymbol{X 1 0}$ & $\boldsymbol{X 1 1}$ \\
\hline $\mathbf{1}$ & +1 & +1 & +1 & +1 & +1 & +1 & +1 & +1 & +1 & +1 & +1 \\
\hline $\mathbf{2}$ & -1 & +1 & -1 & +1 & +1 & +1 & -1 & -1 & -1 & +1 & -1 \\
\hline $\mathbf{3}$ & -1 & -1 & +1 & -1 & +1 & +1 & +1 & -1 & -1 & -1 & +1 \\
\hline $\mathbf{4}$ & +1 & -1 & -1 & +1 & -1 & +1 & +1 & +1 & -1 & -1 & -1 \\
\hline $\mathbf{5}$ & -1 & +1 & -1 & -1 & +1 & -1 & +1 & +1 & +1 & -1 & -1 \\
\hline $\mathbf{6}$ & -1 & -1 & +1 & -1 & -1 & +1 & -1 & +1 & +1 & +1 & -1 \\
\hline $\mathbf{7}$ & -1 & -1 & -1 & +1 & -1 & -1 & +1 & -1 & +1 & +1 & +1 \\
\hline $\mathbf{8}$ & +1 & -1 & -1 & -1 & +1 & -1 & -1 & +1 & -1 & +1 & +1 \\
\hline $\mathbf{9}$ & +1 & +1 & -1 & -1 & -1 & +1 & -1 & -1 & +1 & -1 & +1 \\
\hline $\mathbf{1 0}$ & +1 & +1 & +1 & -1 & -1 & -1 & +1 & -1 & -1 & +1 & -1 \\
\hline $\mathbf{1 1}$ & -1 & +1 & +1 & +1 & -1 & -1 & -1 & +1 & -1 & -1 & +1 \\
\hline $\mathbf{1 2}$ & +1 & -1 & +1 & +1 & +1 & -1 & -1 & -1 & +1 & -1 & -1 \\
\hline
\end{tabular}

\subsubsection{Desenhos Ótimos}

Do inglês Optimal Design é o conjunto de métodos concebidos para construir matrizes de desenho com fatores numéricos e categóricos misturados, com o objetivo de minimizar um conjunto de critérios de avaliação de um desenho [MPB05]. Existem diversos critérios de otimalidade para selecionar os experimentos entre os principais estão A-Optimality que minimiza o traço da inversa da matriz de informação, G-Optimality que minimiza a variança máxima de predição, V-Optimality que minimiza a predição promédio da variança,neste trabalho usamos o criterio de otimalidade $D$ Optimality um dos mais usados na literatura [BP94]. Vale ressaltar que os mencionados critérios dependem de um modelo para a geração dos experimentos nos desenhos [Zey13].

\section{D-Desenho Ótimo:}

D-Optimal Design são otimizações diretas com base em um critério de otimização escolhido e no modelo que será ajustado. O critério de otimalidade usado na geração de desenhos é o de maximizar $\left|X^{\prime} X\right|$, o determinante da matriz de informação $X^{\prime} X$ [Zey13]. Este critério de otimalidade resulta na minimização da variância generalizada das estimativas de parâmetro para um modelo pré-especificado. Como resultado, a otimização de um determinado desenho D-ótimo depende do 
modelo, ver secção 2.11.6. Ou seja, o experimentador deve especificar um modelo para o projeto antes que um computador possa gerar as combinações de tratamento específicas. Dado o número total de execuções de tratamento para um experimento e um modelo especificado, o algoritmo do computador escolhe o conjunto ideal de execuções de design a partir de um conjunto candidato de possíveis execuções de tratamento de design. Esse conjunto candidato de execuções de tratamento geralmente consiste em todas as combinações possíveis de vários níveis de fator que se deseja usar no experimento [BP94].

Em outras palavras, o conjunto candidato é uma coleção de combinações de tratamento a partir das quais o algoritmo D-ótimo escolhe as combinações de tratamento a serem incluídas no projeto. O algoritmo do computador geralmente usa um processo de revisão e troca para selecionar o conjunto de execuções de tratamento.

As razões para usar desenhos D-ótimo em vez de desenhos clássicos padrão geralmente são porque os recursos computacionais são limitados ou se tem um tempo limite para resolver o problema e/ou também quando existe restrições nas configurações dos fatores [MPB05]. Por essas razões foi selecionado este desenho devido que os hyperparametros dos modelos de aprendizagem de maquina apresentavam restrições e que a combinação dos atributos das classes eram numerosos o que complicaria aos recursos computacionais [Zey13].

\section{KL-Exchange}

Muito útil quando os respostas dos pontos do desenho de experimentos dependem de fatores quantitativos e qualitativos, mas também está destinado para trabalhar só com fatores quantitativos, o algoritmo do KL-Exchange surge com o intuito de melhorar a capacidade do algoritmo de Fedorov [Fed13], porque o $K L$-Exchange não avalia todos os possíveis intercâmbios dos pontos do desenho e pontos candidatos, tal que só $k$ pontos de desenhos são considerados para a remoção no desenho, ou seja aqueles com as menores variações de previsão, ademais só L pontos candidatos são considerados para entrar no desenho, ou seja aqueles com as maiores variações de previsão. O KL-Exchange foi inspirado na fórmula de atualização para determinante após a troca de um ponto do desenho por um ponto candidato[AD89].

\subsubsection{Abordagem de exploração do desenho de experimentos}

Dadas as especificações do experimento, uns dos principais passos é gerar o desenho de experimentos é criar um conjunto de pontos candidatos. O conjunto de candidatos é uma tabela de dados com uma linha para cada ponto (corrida) a ser considerado para o desenho. Para o desenho de experimentos com Desenhos Ótimos é necessário definir modelos para gerar os pontos do desenho de experimentos. Os modelos são descrições abstratas do mundo real que entregam uma representação aproximada de funções mais complexas de sistemas físicos [PW00]. A seguinte expressão matemática representa um modelo.

$$
Y\left(x_{i}\right)=\theta^{T} f\left(x_{i}\right)+\epsilon, x_{i} \in \mathbb{R}^{k}, \theta \in \mathbb{R}^{m}
$$

onde $x$ é a variável explanatória, $\theta \in \mathbb{R}^{m}$ é um vector de $m$ parâmetros desconhecidos, $f=$ $\left[f_{1}, \ldots, f_{m}\right]^{T}$ é um vector de $m$ funções continuas definidas num conjunto compacto $X$ que é o rango de regressão tal que $X \subseteq \mathbb{R}^{k}$ e $\epsilon$ denota um processo de erro aleatório [DPZ13]. Estes modelos podem ser definidos por funções lineares, quadráticos ou polinômicos de algum determinado grau que também podem ser combinados com funções com interações e outras funções não lineares[LR07].

\section{Modelos Lineares:}

Os modelos lineares são definidos por funções linearmente independentes, que são representados pela seguinte expressão matemática.

$$
f(x)=a_{n} x_{n}+a_{n-1} x_{n-1}+\ldots+a_{1} x_{1}+a_{0}
$$


onde $a_{n}, a_{n-1}, \ldots, a_{1}$ e $a_{0}$ são números reais com $a_{n} \neq 0$.

\section{Modelos Quadráticos:}

Definidos por funções polinomiais de grau $n=2$, estes funções podem ser definidas pela seguinte expressão:

$$
f(x)=a_{n} x^{n}+a_{n-1} x^{n-1}+\ldots+a_{1} x+a_{0}
$$

onde $a_{n}, a_{n-1}, \ldots, a_{1}$ e $a_{0}$ são números reais com $a_{n} \neq 0$.

\section{Modelos com Interações:}

Uma interação entre $x_{n}$ e $x_{m}$ significa que a relação entre $x_{n}$ e $f(x)$ difere de acordo ao valor de $x_{m}$ e vice versa, uma ventagem é que o modelo é mais flexível, não entanto as vezes é mais difícil de interpretar [TG13], por exemplo uma formula com interação esta representado pela seguinte expressão:

$$
f(x)=a_{n} x_{n} x_{m}+a_{n-1} x_{n-1}+\ldots+a_{1} x_{1}+a_{0}
$$

onde $a_{n}, a_{n-1}, \ldots, a_{1}$ e $a_{0}$ são números reais com $a_{n} \neq 0$, um caso especial pode ser o modelo completo de segundo ordem:

$$
f(x)=a_{5} x_{1}+a_{4} x_{2}+a_{3} x_{1}^{2}+a_{2} x_{2}^{2}+a_{1} x_{1} x_{2}+a_{0}
$$




\section{Capítulo 3}

\section{Coleta de Dados e Pré-processamento}

Para o desenvolvimento deste capítulo, foram utilizados principalmente conceitos de processamento de sinais digitais, conceitos de Variabilidade de Frequência Cardíaca, conceitos de aprendizagem de máquina e teoria de desenho de experimentos. Para ter um panorama de como foram aplicados estes conceitos foi desenhada a seguinte Figura 3.1 que mostra o pipeline do trabalho:

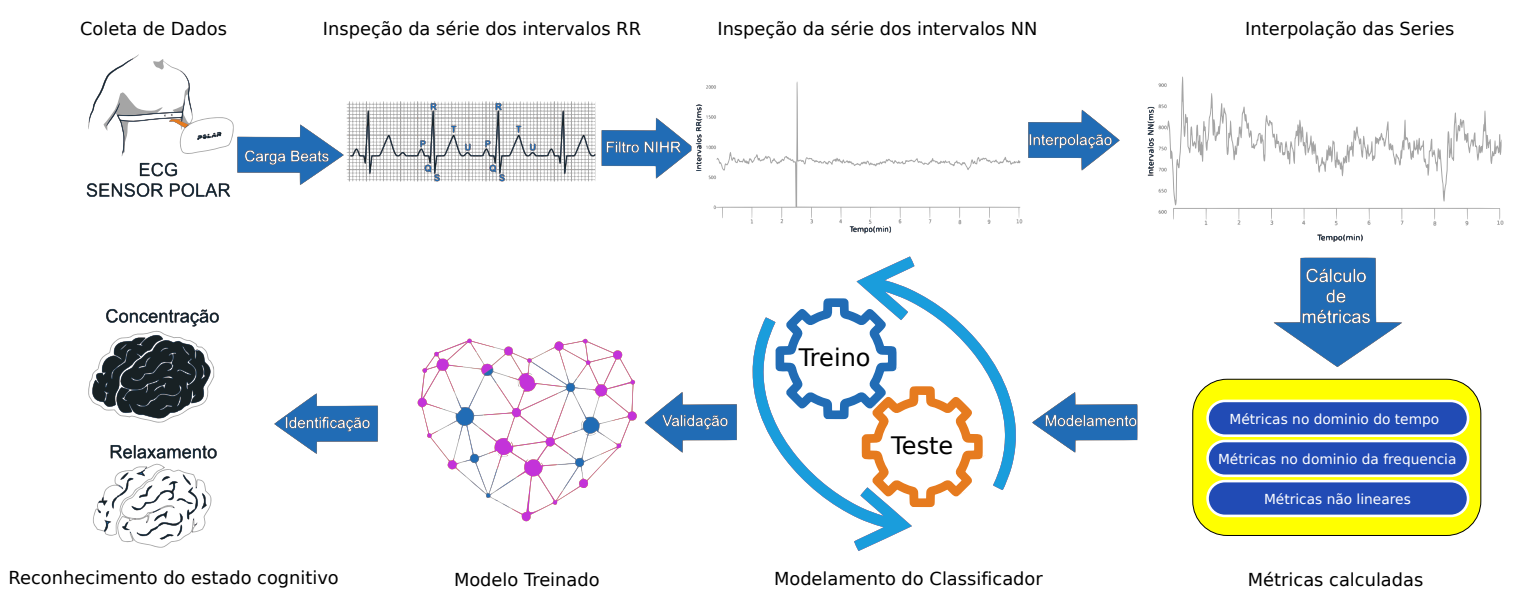

Figura 3.1: Diagrama do pipeline do desenvolvimento do trabalho.

\subsection{Coleta de Dados}

Trinta sujeitos saudáveis destros, todos do sexo masculino, com idade entre 23 e 44 anos participaram deste estudo. Os sujeitos participaram de duas sessões, separadas por aproximadamente uma semana. Coletamos todos os dados antes do meio dia. Para a coleta de dados na segunda sessão a maioria delas foram realizadas aproximadamente no mesmo horário que a primeira.

Excluímos cinco sujeitos devido à declaração de desconforto, nervosismo, ou porque tinham consumido cafeína. Assim, nosso conjunto de dados final foi composto de 25 sujeitos. Nenhum dos 25 sujeitos apresentava doença cardiorrespiratória, não usavam drogas ou remédios, nem tinham feito exercícios exaustivos 24 horas antes da coleta de dados. Todos eles dormiram aproximadamente 
oito horas na noite anterior à coleta de dados. O protocolo do experimento foi aprovado pelo Comitê de Ética em Pesquisa em Humanos da Escola de Artes, Ciências e Humanidades (EACH) da Universidade de São Paulo (USP). A Figura 3.2 descreve as componentes usadas dentro da arquitetura do sistema de coleta e processamento de dados.

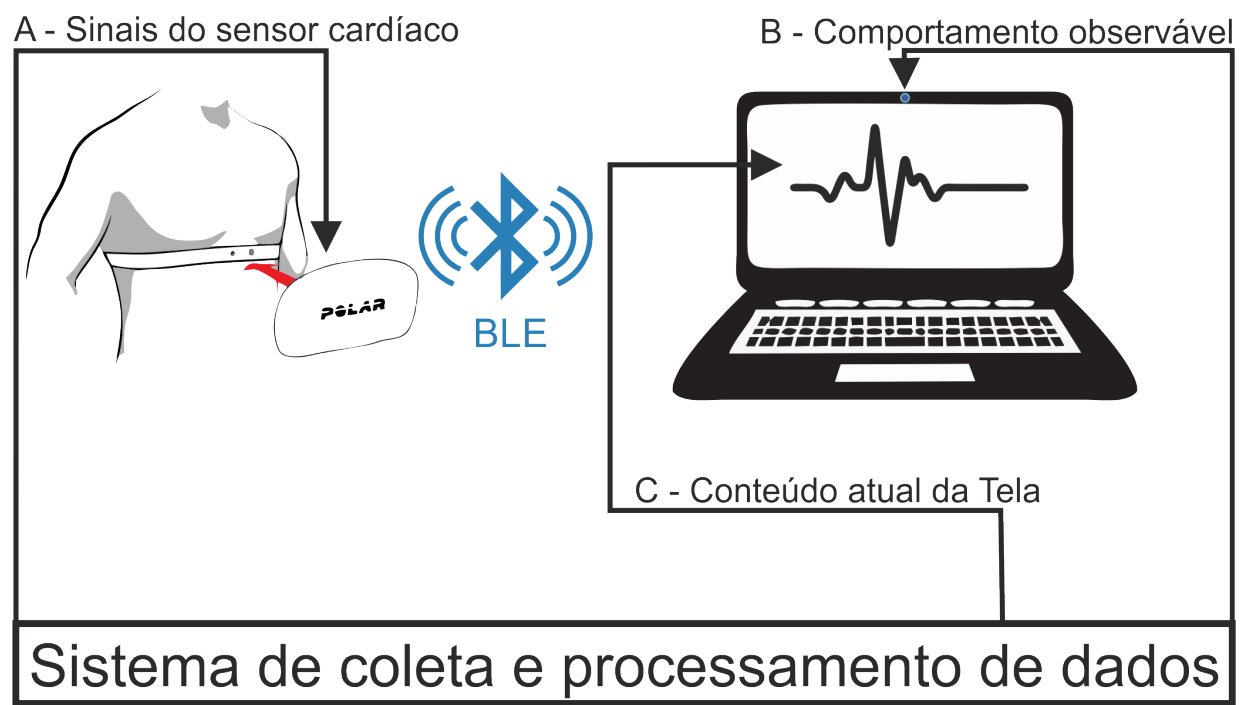

Figura 3.2: Configuração do experimento. (A) Registro de sinais cardíacos com o sensor Polar H10; (B) Registro de vídeo do comportamento dos usuários, ações observáveis dos usuários (expressão facial, postura e gestos) e (C) Registro de vídeo do conteúdo atual da tela.

\subsubsection{Aplicativo de coleta de dados}

Para realizar a coleta de dados foi desenvolvido uma aplicação desktop, onde a linguagem selecionada foi Python pela capacidade de integração e compatibilidade entre as bibliotecas de manipulação do sensor e as bibliotecas das técnicas de aprendizado de máquina. $\mathrm{O}$ aplicativo permitiu o registro dos dados para cada indivíduo nas diferentes atividades cognitivas. O aplicativo armazenou as séries temporais num banco de dados de arquivos (CSV, do inglês Comma Separated Value), durante um tempo determinado de 10 minutos. Além disso, mostra de forma paralela o comportamento das seguintes métricas: Heart Rate, rMSSD, pNN50, HRSTD, SDNN, MeanRR, LF, HF, LFHF, Power, FracDim, num tamanho de janela de 45 segundos para cada métrica. A conexão entre o sensor Polar H10 e o computador é feita mediante a tecnologia de comunicação (BLE, do inglês Bluetooth Low Energy) ${ }^{1}$, um tipo de comunicação inteligente, porque a transmissão dos dados coletados pelo sensor são ativadas quando o sensor está ligado, ou seja quando os electrodos da fita estão em contato com a pele, o que permite um menor consumo de energia [Sod19].

O sensor tem diferentes serviços, tal que dentro de cada serviço há características diferentes que podem ser utilizadas. A tecnologia BLE do sensor Polar H10 usa os Serviços Bluetooth (GATT, do inglês General Attributes) indicados na Tabela 3.1:

Tabela 3.1: Serviços Bluetooth GATT usados pelo Sensor Polar $\mathrm{H}^{2}{ }^{2}$.

\begin{tabular}{l|l|l} 
Device Information(0x180A) & Battery Service(0x180F) & Heart Rate Service(0x180D) \\
\hline Manufacturer Name String & Battery level & Heart Rate Measurement \\
Model Number String & & Body Sensor Location \\
Serial Number String & & Heart Rate Control Point
\end{tabular}

No âmbito deste trabalho a característica usada foi a Heart Rate Measurement (0x2A37) [LY13], que têm os seguintes campos disponíveis:

\footnotetext{
${ }^{1}$ https://www.bluetooth.com/specifications/specs/

${ }^{2}$ https://github.com/polarofficial/polar-ble-sdk
} 
- Gravação da frequência cardíaca (bpm)

- Gravação dos intervalos RR (ms)

- Indicador de contato do sensor

Mediante o aplicativo são capturados a frequência cardíaca e os intervalos RR ao mesmo tempo, para calcular em tempo real as métricas da HRV. Dentre as métricas no domínio do tempo foram selecionadas: mrr, mhr, sdnn, cv_rr, rmssd, hrstd e pNN50. Dentre as métricas no domínio da frequência foram selecionadas: ULF power, VLF power, LF power, HF power, Power e LF/HF. No caso das métricas não lineares foram selecionadas: ApEn e FracDim. Para o cálculo deste conjunto de métricas foi usado o gHRV $\left[\mathrm{RLLV}^{+} 14\right]$ um pacote de software (toolbox), disponível em Python.

Real Time Capture Data HRV

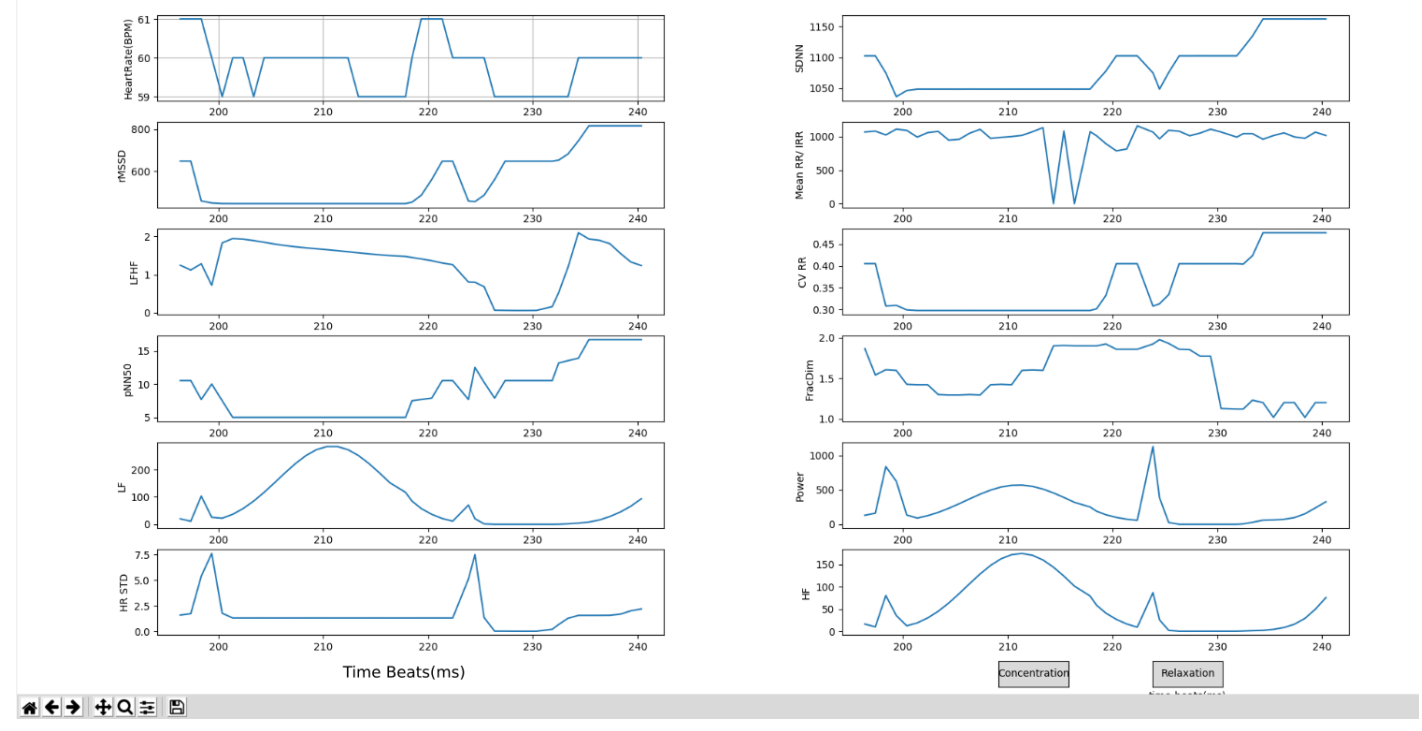

Figura 3.3: Aplicativo de Coleta de Dados com as séries temporais das métricas da HRV dos últimos 45 dados recebidos do sensor com as opções para o usuário registrar uma sessão de atividade.

Diversos toolbox para a análise da HRV foram avaliados, mediante um análise comparativo entre estas ferramentas, o gHRV ofereceu principalmente o cálculo das métricas no domínio do tempo, no domínio da frequência e não lineares, além disso ofereceu maior compatibilidade com a leitura dos dados entregues pelo sensor cardíaco, devido ao módulo dedicado para a leitura do seus dados com uma Interface Gráfica do Usuário disponível para a visualização dos dados. Outra ventagem deste toolbox é que presenta uma documentação mais completa e o código fonte aberto em comparação com outros toolbox, o que permite uma maior escalabilidade com a integração de outras aplicações e implementações de novas métricas da HRV, como por exemplo as métricas geométricas.

\subsubsection{Protocolo de coleta de dados}

A coleta de dados foi feita em duas sessões em ambientes controlados, mantendo principalmente um controle sobre o mesmo horário entre as sessões dos diferentes dias, sobre as condições de descanso do participante (que deve ter sido pelo menos 8 horas na noite anterior), sobre o estado físico e mental do participante e sobre não ter consumido cafeína as últimas 12 horas pelo menos. Todo o mencionado foi perguntado para o participante antes de iniciar com a sessão de coleta de dados, para avaliar se ele estava em condições de começar com o experimento. Depois de ter avaliado aos participantes, pedimos para eles que se sentassem em ângulo reto e fechassem os olhos durante todas as tarefas solicitadas, estas tarefas foram quatro, tal que são as seguintes:

- Estado de repouso: Os participantes foram instruídos a ficar em repouso, mantendo a concentração na respiração. 
- Tarefa de memória (A): Os participantes foram instruídos a lembrar dos eventos que ocorreram desde o dia anterior até o momento do experimento.

- Tarefa de canto mental (B): Os participantes foram instruídos a cantar suas músicas favoritas mentalmente.

- Tarefa de subtração (C): Os participantes foram instruídos a contar de forma decrescente desde 5000 de sete em sete.

Estas atividades cognitivas foram extraídas e adaptadas do [SRR $\left.{ }^{+} 12\right]$, as quais foram executadas por um tempo de 10 minutos cada. A primeira tarefa em todas as sessões sempre foi o estado de repouso, recomendável para estabelecer uma linha de base segundo [QH14]. Em seguida, as demais tarefas foram realizadas de forma aleatória e contrabalanceadas ao longo de todos os participantes. Na Tabela 3.2 é possível ver a quantidade de registros em média que foi coletado para cada atividade cognitiva com seu respetivo desvio padrão nas diferentes sessões. Isto foi perto de 600 registros por cada sessão, porque o sensor retorna os dados numa frequência de um $1 \mathrm{~Hz}$.

Tabela 3.2: Média e Desvio Padrão da quantidade de registros de dados coletados para cada tarefa cognitiva nas diferentes sessões.

\begin{tabular}{lcccc|cccc}
\cline { 2 - 8 } & \multicolumn{4}{c|}{ Sessão 1 } & \multicolumn{4}{c}{ Sessão 2 } \\
\cline { 2 - 8 } & Repouso & Música & Memória & Subtração & Repouso & Música & Memória & Subtração \\
\hline Média & 604.72 & 538.48 & 515.04 & 590.12 & 598.2 & 517.32 & 569.56 & 535.24 \\
\hline Desv. Padrão & 66.69 & 102.42 & 137.47 & 70.38 & 84.28 & 106.90 & 67.40 & 93.33 \\
\hline
\end{tabular}

\subsection{Carregamento e observação dos dados}

Nesta etapa a data coletada foi carregada e organizada por cada tarefa cognitiva e por cada participante nas diferentes sessões. Como os principais dados obtidos direitamente pelo sensor são a HR e os intervalos RR, na Figura 3.4 se mostra um histograma com a média e desvio padrão do comportamento de essas métricas por cada sessão e atividade cognitiva. Para cada tarefa cognitiva

A)

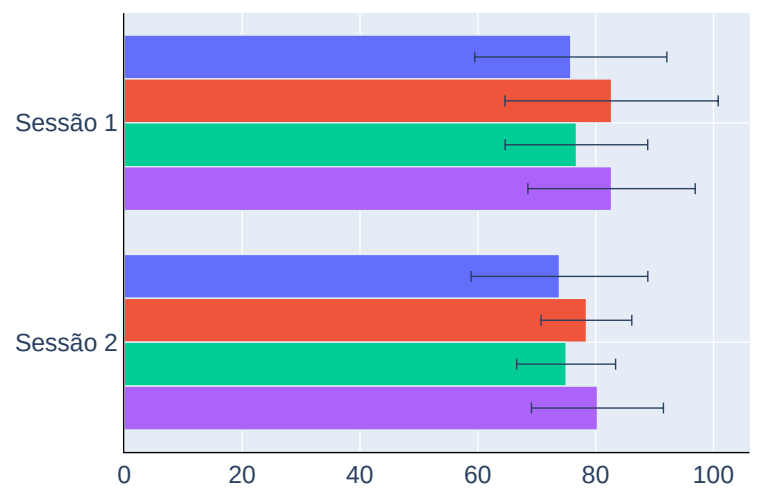

Média da HR nas diferentes atividades cognitivas
B)

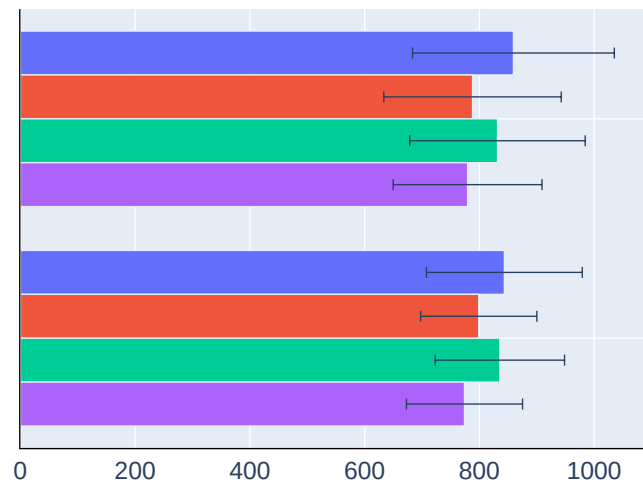

- Repouso

- Memória

- Música

- Subtração

Figura 3.4: Média da HR e dos intervalos $N N$ de todos os participante nas diferentes atividades cognitivas em cada tarefa.

foram calculadas as seguintes métricas MRR, MHR, SDNN, CV_RR, rMSSD, HRSTD, pNN50, ULF power, VLF power, LF power, HF power, Total Power e LFHF, ApEn, FracDim a partir dos intervalos RR e a frequência cardíaca (HR). Uma vez organizado os dados, para cada atividade 
cognitiva foi removido os 50 primeiros registros e os 50 últimos registros em cada, para desta forma limpar e obter exatamente os registros das atividades executadas.

Depois observamos preliminarmente o comportamento dos dados por cada individuo como é sugerido em [KK06] como parte do pré-processamento, o que permite um melhor entendimento dos dados. Como por exemplo na Figura 3.5 podemos diferenciar parcialmente as 4 atividades cognitivas mediante os intervalos NN.

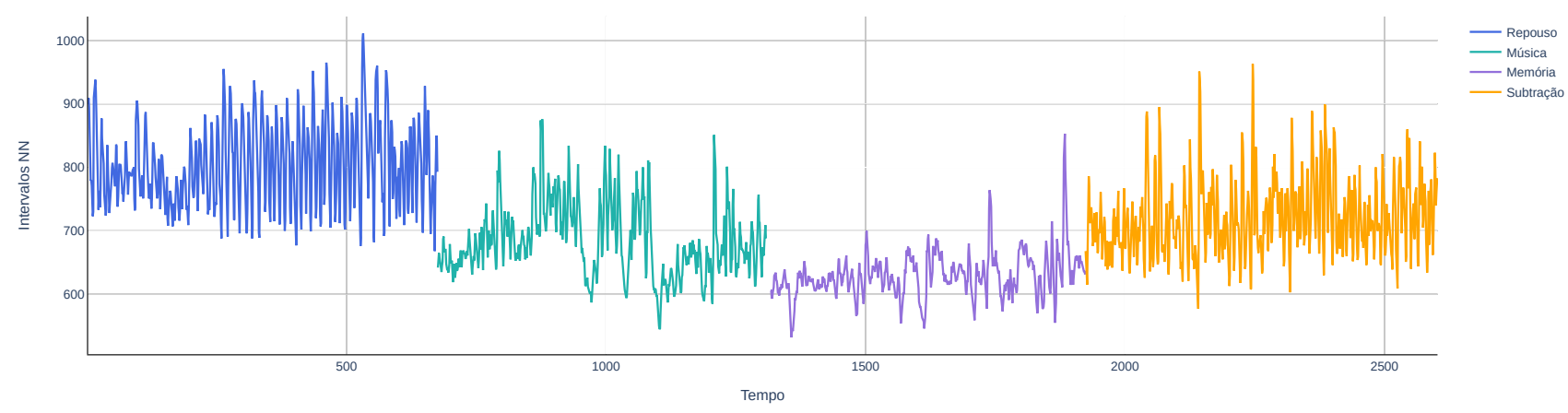

Figura 3.5: Serie do tempo dos intervalos NN para um participante nas diferentes atividades cognitivas

Também foi possível perceber padrões nas métricas no domínio da frequência, em alguns participantes, por exemplo na Figura 3.6 mostra os espectros de potencia no periodograma de Welch [Wel67] dos intervalos RR normalizados entre as sessões de dias diferentes. Por exemplo, no painel "Repouso 1" e "Subtrair 1" correspondem à primeira sessão e o "Repouso 2" e "Subtrair 2" correspondem à sessão 2. Assim, é possível perceber similaridades nos espectros de potência, das diferentes sessões, para um mesmo participante numa mesma tarefa, especialmente as similaridades foram encontradas no espectro da potencia baixa (LF power), cor laranja; também mostra uma diferença marcada entre as diferentes atividades cognitivas.

\subsection{Filtragem dos dados}

Depois de ter coletado os dados com o sensor Polar $\mathrm{H}_{10}{ }^{3}$, especificamente as series dos intervalos RR e da Frequência Cardíaca, foi necessário fazer a limpeza dos intervalos RR atípicos para assim obter os intervalos NN ou intervalos RR normalizados.

Para remover o ruído na série da Frequência Cardíaca, aplicamos um filtro nos dados. Este processo consistiu primeiramente em remover todos os valores de Frequência Cardíaca que estão fora dos limites do intervalo $24 \leq H R \leq 198$ que consideramos aceitáveis, baseado no estudo de [OOC86]. Estes limites foram estabelecidos na execução do pipeline e depois os valores eliminados foram completados com uma interpolação linear.

Depois, o filtro aplicado na serie dos intervalos RR consistiu em remover aqueles que estavam fora dos limites do intervalo $300 \leq I R R \leq 2000$. Depois de ter aplicado o filtro foi necessária a interpolação linear para completar os valores atípicos (outliers) removidos. Imediatamente, foi aplicado um filtro conhecido como método do Malik [KF95, ASHM00], desenhado para eliminar batimentos ectópicos na medição da HRV de curto prazo, ele é descrito melhor no Algoritmo 3.7, finalmente é aplicado uma interpolação linear para substituir os valores ectópicos removidos. Os batimentos cardíacos ectópicos são alterações em um batimento cardíaco que, de outra forma, é normal, produzidos geralmente por um a um batimento cardíaco prematuro. O ritmo ectópico também é conhecido como contração atrial prematura, contração ventricular prematura e extrasístole [KHA07]. E os valores atípicos outliers são valores mais extremos que os valores ectópicos,

\footnotetext{
${ }^{3}$ https://www.polar.com/br
} 

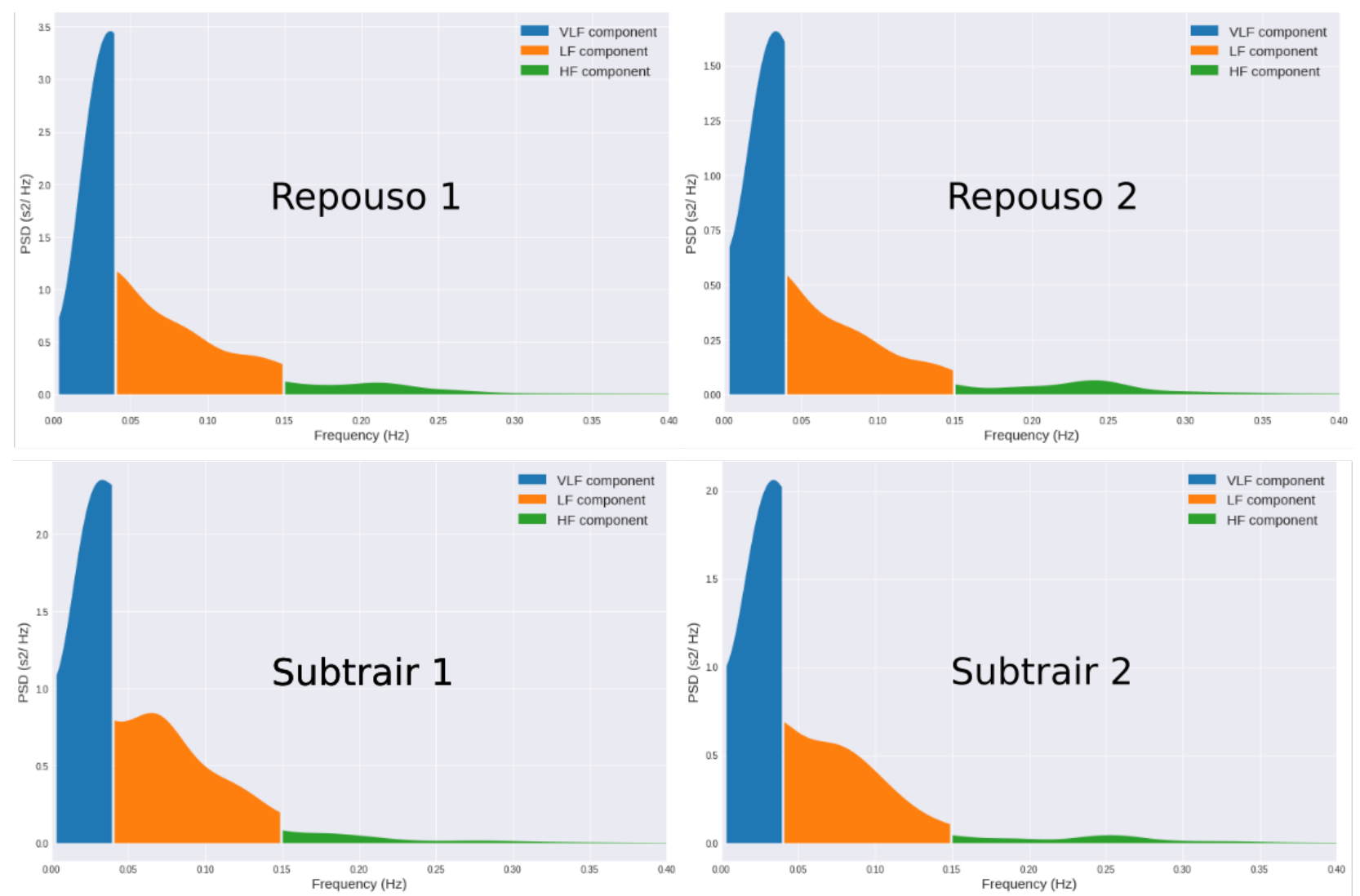

Figura 3.6: Espectro da potencia do sinal dos intervalos $N N$ mostrando as bandas de frequência (VLF, LF e HF) para sessões de 10 minutos em diferentes dias.

usualmente são gerados por um mal contato com os eletrodos do sensor, bateria baixa, interferência na sinal para recuperar o sinal elétrico da despolarização do coração e outras questões próprias do sensor.

Uma vez filtrado e limpo de ruido, os intervalos RR serão chamados de intervalos NN, ou também conhecidos como intervalos RR normalizados.

Na Figura 3.8-A é possível ver uma serie de intervalos RR obtida direitamente do sensor e na Figura 3.8-B a mesma serie com os filtros aplicados com as técnicas mencionadas para obter os intervalos NN. Para esta etapa de preprocessamento foi usado o toolbox hrv-analysis [Cha21].

\subsection{Interpolação dos intervalos NN}

Após obter a série de intervalos NN, foi definido uma janela deslizante de tamanho 45 segundos, que percorre a série dos intervalos NN com um deslocamento $d$ inicial de 1 registro, como se pode ver na Figura 3.9. Foi necessário fazer a interpolação dos dados por cada janela por dois motivos principais: primeiro o sensor tem uma taxa de amostragem de $1000 \mathrm{~Hz}$ [GDN16] para a coleta de dados, mas ele processa os dados coletados e os entrega a uma frequência de $1 \mathrm{~Hz}$. Segundo, o toolbox $\left[\mathrm{RLLV}^{+} 14\right]$ recomenda e requer que as janelas estejam reamostradas para poder calcular principalmente as métricas da HRV no domínio da frequência. Assim, uma reamostragem de 4.0 $\mathrm{Hz}$ foi feita mediante uma interpolação linear, como é recomendado em diversos trabalhos [Cli02, SVS04], para analisar o HRV e calcular assim suas métricas de forma confiável, principalmente nas métricas do domínio da frequência as que são calculadas mediante a Transformada Rápida de Fourier (FFT, do inglês Fast Fourier Transform), método de análise espectral não paramétrica. 
Figura 3.7: Método do Malik para encontrar valores ectópicos nos intervalos $R R$
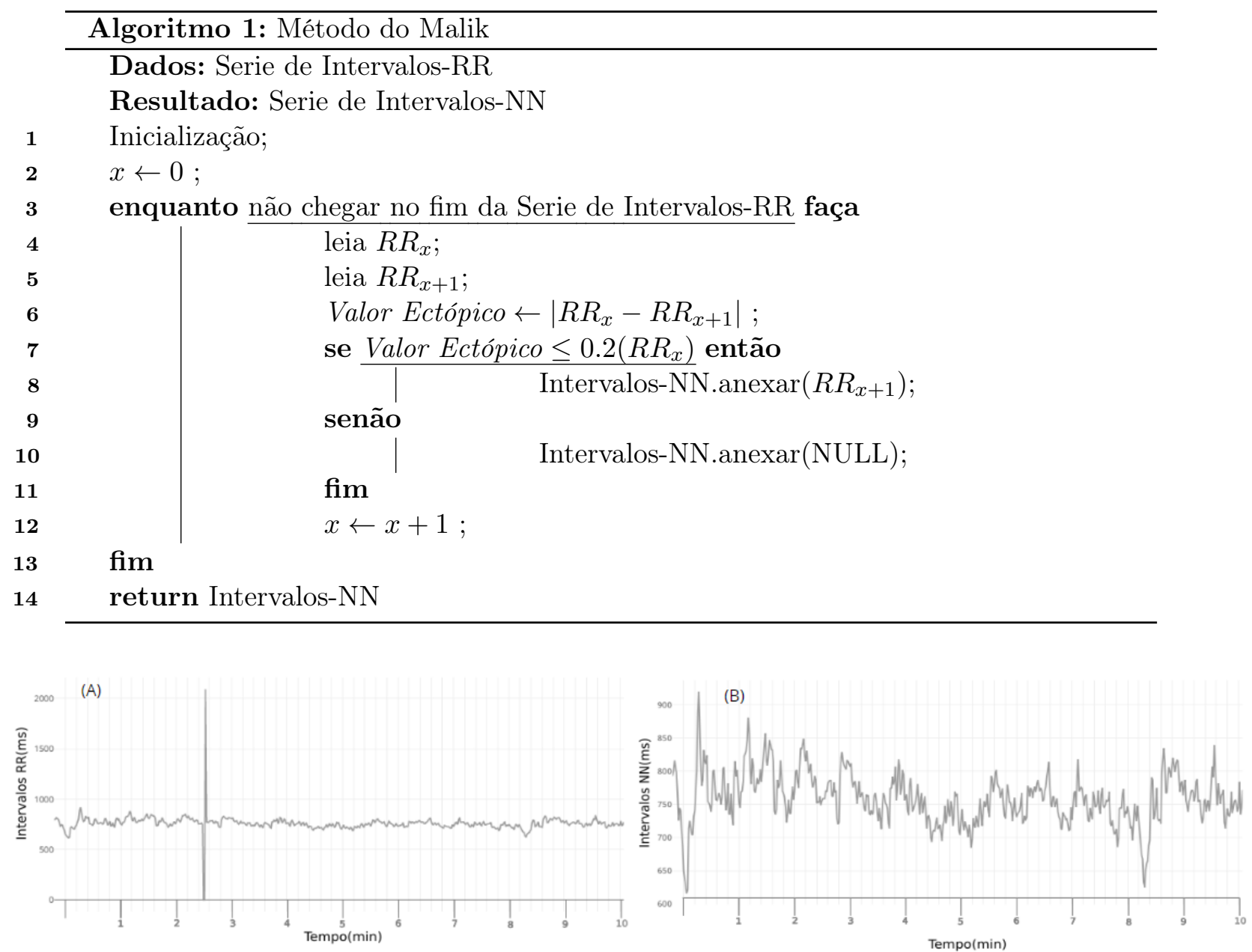

Figura 3.8: (A) Intervalos $R R$ na tarefa cognitiva de lembrança e (B) o filtro e interpolação aplicado na serie (A) para obter os Intervalos $N N$ na tarefa cognitiva de lembrança, as series tem um tempo de coleta de 10 min.

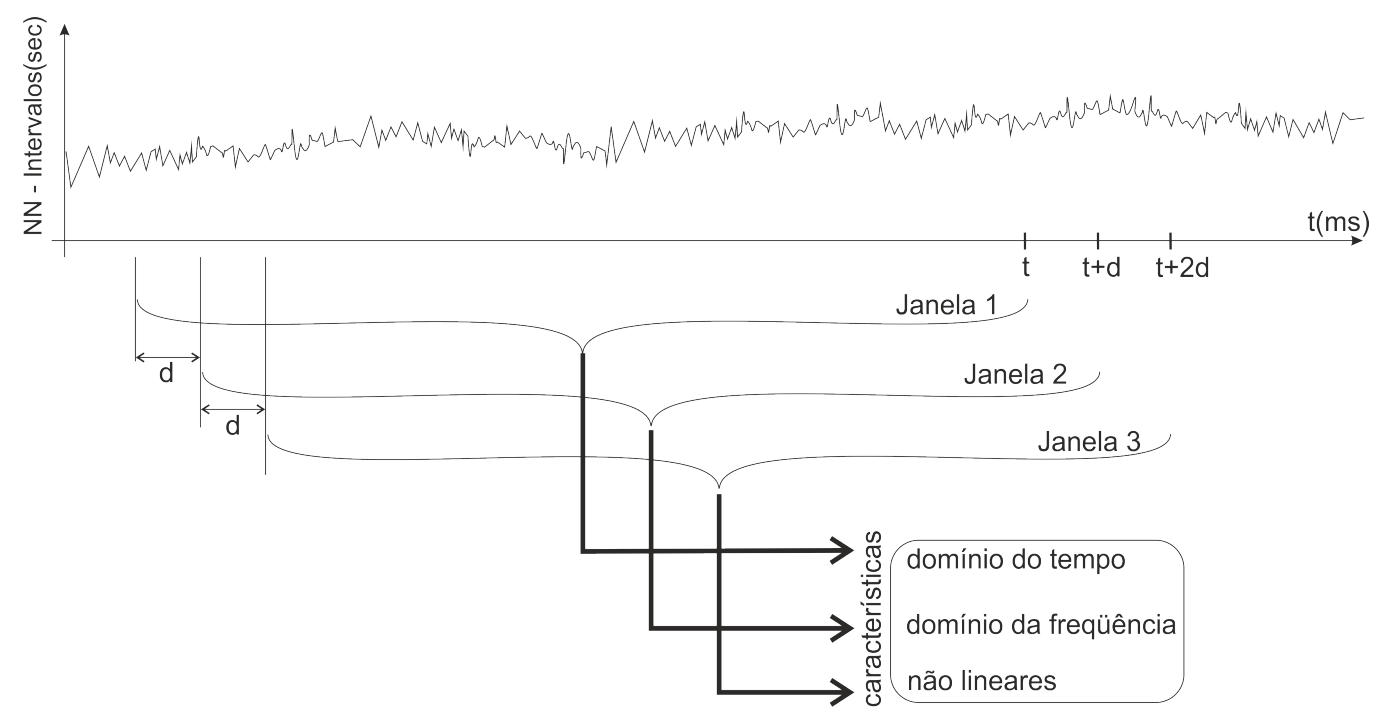

Figura 3.9: Interpolação por janelas deslizantes sobre a serie dos intervalos NN, com um deslocamento de tamanho d para janelas de tamanho fixo, para depois calcular as métricas da HRV por cada janela. 


\subsection{Cálculo das métricas da HRV}

Uma vez obtido os dados pré-processados e livres de ruído, realizamos, para cada janela, o cálculo das métricas da HRV no domínio do tempo, no domínio da frequência e as não lineares. O cálculo das métricas foi realizado com ajuda do toolbox gHRV [RLLV+14]. As métricas indicadas na Tabela 3.3 junto com os intervalos NN e a frequência cardíaca, serviram como um conjunto de dados para treinar os classificadores.

Tabela 3.3: Métricas calculadas para cada janela nas diferentes sessões e usadas para a classificação

\begin{tabular}{l|l|l} 
Domínio do tempo & Domínio da Frequência & Não Lineares \\
\hline pnn50 & hf & \\
mrr & lf & ApEn \\
cv_rr & vlf & FracDim \\
rmssd & ulf & \\
hrstd & lfhf & \\
sdnn & power &
\end{tabular}

mhr 


\section{Capítulo 4}

\section{Experimentos e análise de resultados}

\subsection{Modelagem do classificador}

Nesta seção foram avaliados o desempenho de diferentes modelos de classificação baseados principalmente nos algoritmos de Support Vector Machine (SVM), Random Forest (RF), Logistic Regression (LR) e Decision Tree (DT). Para isso foi importante seguir um conjunto de passos preliminares que ajudaram na análise, começando com a codificação dos rótulos de cada classe, normalização dos dados, identificação das métricas que mais influenciam o classificador, a divisão dos dados em treino e teste, treinamento dos diferentes modelos, hiper-parametrização dos modelos escolhidos baseado nas métricas de desempenho do classificador, validação, re-treinamento dos modelos e finalmente testar o modelo para garantir que funciona para dados independentes.

\subsubsection{Normalização dos dados}

No contexto de abordagens de preprocessamento, normalizar significa colocar todos os dados na mesma escala para que cada métrica tenha uma contribuição similar no modelagem do classificador, o tipo de normalização usado nesta etapa foi o Padrão ou também conhecido como normalização ZScore, que tem a propriedade de ser menos sensível ao ruido [SS20] e é dado da seguinte forma, seja $X_{i}$ um valor que pertence ao atributo $X, X_{\text {média }}$ a média dos valores de $X$ e $X_{\text {desvio_padrão o desvio }}$ padrão dos valores de $X$ [FLZH19], então o dado normalizado é:

$$
X_{\text {normalizado }}=\frac{X_{i}-X_{\text {média }}}{X_{\text {desvio_padrão }}}
$$

Entre as principais vantagens da normalização estão a melhora em velocidade do processo de treinamento dos dados e principalmente minimizar o viés que pode induzir alguma métrica [NMB14].

Como parte do preprocessamento dos dados também foi necessário codificar as variáveis categóricas para um treinamento mais rápido dos modelos, além de que muitos modelos de ML só aceitam variáveis numéricas [PP17], tal que as diferentes classes (Repouso, Memória, Canto, Subtrair) foram correspondidas com a tupla de valores $(0,1,2,3)$.

\subsubsection{Casos de análise}

Foram definidos 22 casos de análise derivados da combinação das diferentes classes, o primeiro conjunto de 11 casos de análise foram baseadas no análise de classificação intradiaria e os outro 11 casos de análise no análise de classificação interdiaria, estes casos de análise foram detalhados na Tabela 4.1, com a finalidade de encontrar quais das tarefas cognitivas são mais diferenciadas umas das outras. Dentro de cada caso de análise foram preparados os dados de treino e validação, o que representa um passo muito importante no desenvolvimento do pipelines de Aprendizagem de Máquina, porque ajudou na organização dos dados para o modelamento do classificador. 
Tabela 4.1: Casos de análise agrupando as diferentes atividades cognitivas no mesmo dia e em dias diferentes.

\begin{tabular}{|l|l|}
\hline Número do Caso & \multicolumn{1}{c|}{ Caso de Análise } \\
\hline Caso 1 & 4 Classes intradiário (repouso,memória,música,subtração) \\
\hline Caso 2 & 3 Classes intradiário (repouso,memória,subtração) \\
\hline Caso 3 & 3 Classes intradiário (memória,subtração,música) \\
\hline Caso 4 & 3 Classes intradiário (repouso,música,subtração) \\
\hline Caso 5 & 3 Classes intradiário (repouso,música,memória) \\
\hline Caso 6 & 2 Classes intradiário (repouso,subtração) \\
\hline Caso 7 & 2 Classes intradiário (repouso,memória) \\
\hline Caso 8 & 2 Classes intradiário (repouso,música) \\
\hline Caso 9 & 2 Classes intradiário (memória,música) \\
\hline Caso 10 & 2 Classes intradiário (subtração,música) \\
\hline Caso 11 & 2 Classes intradiário (subtração,memória) \\
\hline Caso 12 & 4 Classes interdiário (repouso,memória,música,subtração) \\
\hline Caso 13 & 3 Classes interdiário (repouso,memória,subtração) \\
\hline Caso 14 & 3 Classes interdiário (memória,subtração,música) \\
\hline Caso 15 & 3 Classes interdiário (repouso,música,subtração) \\
\hline Caso 16 & 3 Classes interdiário (repouso,música,memória) \\
\hline Caso 17 & 2 Classes interdiário (repouso,subtração) \\
\hline Caso 18 & 2 Classes interdiário (repouso,memória) \\
\hline Caso 19 & 2 Classes interdiário (repouso,música) \\
\hline Caso 20 & 2 Classes interdiário (memória,música) \\
\hline Caso 21 & 2 Classes interdiário (subtração,música) \\
\hline Caso 22 & 2 Classes interdiário (subtração,memória) \\
\hline
\end{tabular}

Com os dados de treino ajustamos os modelos, enquanto os dados de validação nos serviram para calcular o erro dos modelos, mediante a avaliação dos modelos treinados, a Figura 4.5 e a Figura 4.2 mostram a média das acurácias dos 25 participantes por cada caso de análise, de forma ordenada, onde para o Caso 12 se obteve as acurácias mais baixas, e no Caso 7 se obteve as acurácias mais altas, estes resultados foram antes do ajuste de hiper-parâmetros e a seleção de métricas da HRV.

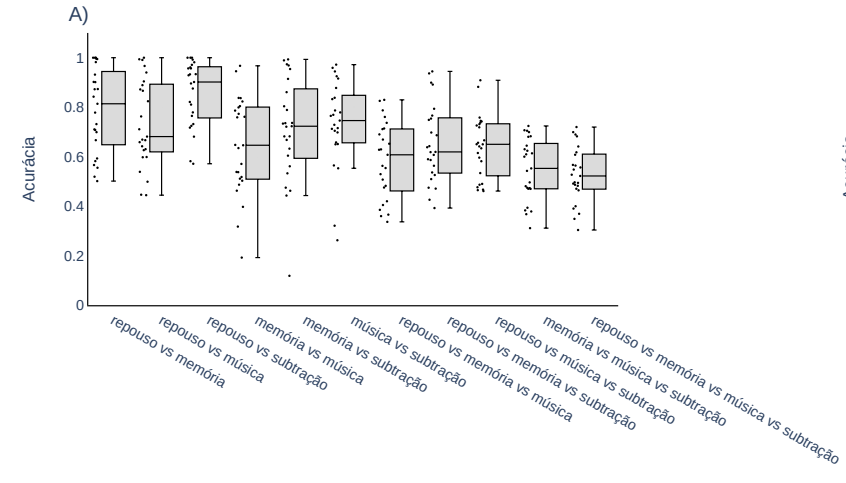

Casos de análise

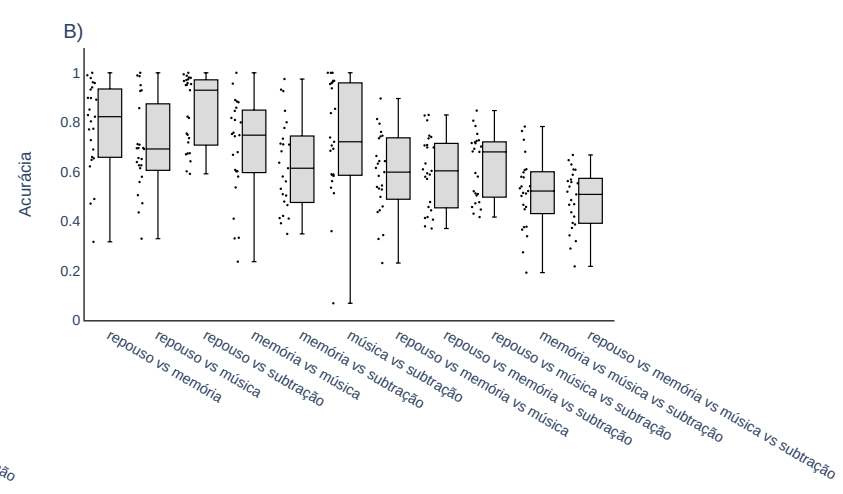

Casos de análise

Figura 4.1: Acurácias de classificação de tarefas cognitivas intradiárias para o SVM. (A) Obtivemos a acurácia da classificação usando a primeira metade dos dados do dia 1 como o conjunto de treinamento e a segunda metade como o conjunto de validação. (B) Obtivemos a acurácia da classificação usando a primeira metade dos dados do dia 2 como o conjunto de treinamento e a segunda metade como o conjunto de validação. 


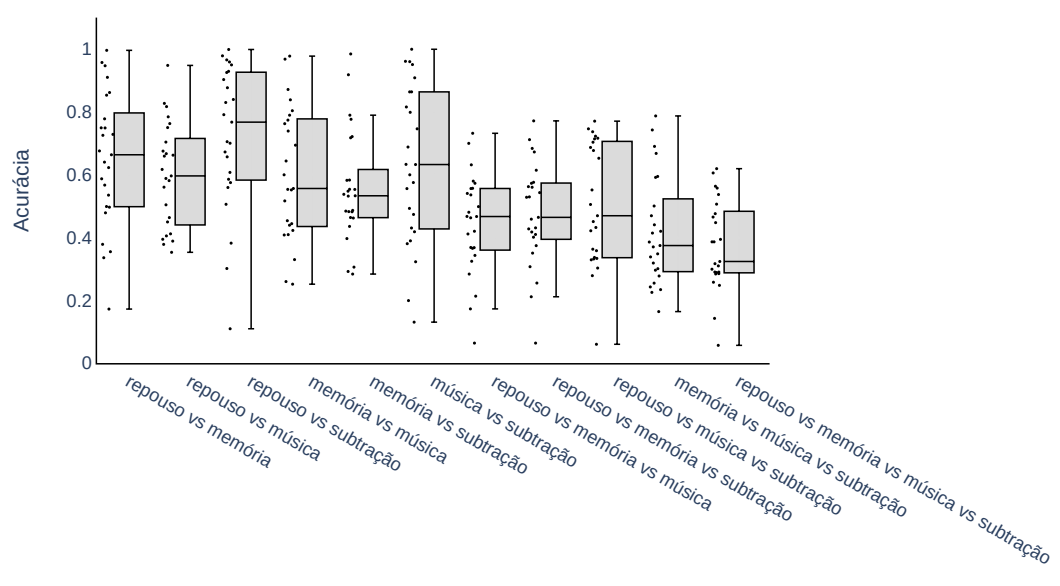

Casos de análise

Figura 4.2: Acurácias de classificação de tarefas cognitivas interdiárias. Usamos os dados do dia 1 como o conjunto de treinamento e os dados do dia 2 como o conjunto de validação.

\subsection{Especificação de espaço de busca de hiperparâmetros}

Os espaços de busca são o conjunto de configurações de hiperparâmetros combinadas para avaliar o comportamento dos hiperparâmetros com base na acurácia dos diferentes modelos de classificação dos algoritmos (SVM, DT, RF, DT). Para poder avaliar a eficácia dos modelos hiperparâmetrizados, os modelos base foram definidos para cada experimento. Esses modelos base são modelos com os hiperparâmetros padrão fornecidos pelo Scikit-Learn [FPea11]. A especificação do espaço de busca e o tempo de execução base para o algoritmo SVM são especificados na Tabela 4.2, para o DT na Tabela 4.3, para o RF na Tabela 4.4 e para o LR na Tabela 4.5. Nas tabelas mencionadas a primeira coluna mostra os hiperparâmetros para cada algoritmo que foram ajustados, a segunda coluna mostra a quantidade de valores que pode assumir o hiperparâmetro, a terceira coluna mostra o tamanho total de execuções definidas no espaço de busca, a quarta coluna mostra o tempo base em segundos requerido por cada execução e a quinta coluna mostra o total de horas.

Para manter padronizado o tamanho do espaço de busca nos diferentes algoritmos, inicialmente foram selecionados 120 amostras aleatórias do desenho de experimentos gerado para cada algoritmo em cada participante e cada caso de análise. Por exemplo no SVM foi requerido aproximadamente 10.4 horas para os 25 participantes num caso de análise, esse tempo aproximado teve que ser replicado para os 22 casos de análise, para o qual foi necessário da computação de alto desempenho para poder ajustar melhor os hiperparâmetros em cada situação específica, e assim foi feito para os outros algoritmos.

Tabela 4.2: Especificação do espaço de busca do SVM definido para o ajuste de hiperparâmetros ${ }^{1}$.

\begin{tabular}{lcccc}
\hline SVM & Valores & Tamanho Total & $\begin{array}{c}\text { Tempo Base } \\
\text { em Segundos }\end{array}$ & Total Horas \\
\hline C & 10 & & & \\
kernel & 4 & & & 12.48 \\
gamma & 2 & 120 & & \\
cache_size & 4 & & & \\
class_weight & 2 & & & \\
\hline
\end{tabular}

\footnotetext{
${ }^{1}$ https://scikit-learn.org/stable/modules/generated/sklearn.svm.SVC.html

${ }^{2}$ https://scikit-learn.org/stable/modules/generated/sklearn.tree.DecisionTreeClassifier.html

${ }^{3}$ https://scikit-learn.org/stable/modules/generated/sklearn.ensemble.RandomForestClassifier.html

${ }^{4}$ https://scikit-learn.org/stable/modules/generated/sklearn.linear_model.LogisticRegression.html
} 
Tabela 4.3: Especificação do espaço de busca do DT definido para o ajuste de hiperparâmetros ${ }^{2}$.

\begin{tabular}{lcccc}
\hline DT & Valores & Tamanho Total & $\begin{array}{c}\text { Tempo Base } \\
\text { em Segundos }\end{array}$ & Total Horas \\
\hline criterion & 2 & & \\
max_features & 17 & 120 & 10.25 & 8.54 \\
min_samples_split & 3 & & \\
min_samples_leaf & 3 & & \\
max_depth & 10 & & \\
\hline
\end{tabular}

Tabela 4.4: Especificação do espaço de busca do RF definido para o ajuste de hiperparâmetros ${ }^{3}$.

\begin{tabular}{lcccc}
\hline RF & Valores & Tamanho Total & $\begin{array}{c}\text { Tempo Base } \\
\text { em Segundos }\end{array}$ & Total Horas \\
\hline n_estimators & 200 & & \\
criterion & 2 & & \\
max_features & 17 & 120 & 12.0 \\
min_samples_split & 3 & & \\
min_samples_leaf & 3 & & \\
max_depth & 10 & & \\
\hline
\end{tabular}

Tabela 4.5: Especificação do espaço de busca do LR definido para o ajuste de hiperparâmetros ${ }^{4}$.

\begin{tabular}{lcccc}
\hline LR & Valores & Tamanho Total & $\begin{array}{c}\text { Tempo Base } \\
\text { em Segundos }\end{array}$ & Total Horas \\
\hline penalty & 4 & 120 & 11.40 & 9.5 \\
C & 10 & & \\
class_weight & 2 & & \\
solver & 5 & & & \\
\hline
\end{tabular}




\subsection{Desempenho no ajuste de hiperparâmetros}

Nesta etapa do trabalho usamos uma biblioteca de software conhecida como ExperimentalDesign.jl [Bru20] criada para o Desenho de Experimentos (DOE), que contém implementações de vários métodos DOE, tais como desenhos fatoriais, desenhos de Plackett-Burman, desenhos aleatórios e desenhos ótimos, este toolbox foi desenvolvido na linguagem Julia que tem como principal vantagem presentar um melhor rendimento para execuções que exigem muito poder computacional, o que foi útil no nosso trabalho para a construção das matrizes dos espaços de busca e para encontrar a melhor combinação de hiperparâmetros nos diferentes participantes ao longo de todos os casos de análise definidos. A partir da definição desses espaços de busca dos hiperparâmetros, os delineamentos de experimentos foram criados com a técnica D-Optimal Design e a estratégia $K L$ Exchange [AD89], uma variação dos desenhos ótimos.

Para a construção dos desenhos ou matrizes experimentais com a técnica D-Optimal Design é necessário definir modelos de hipóteses de comportamento dos fatores ou hiperparâmetros dos diferentes algoritmos de aprendizado de máquina, nesta etapa usamos modelos lineares e modelos com interações [Zey13].

D-Optimal Design foi escolhido por que os desenhos factoriais requeriam muito tempo em execuções e os fatores de análise neste caso os hiperparâmetros estavam restritos por intervalos de possíveis valores onde foi necessário otimizar maximizar o ganho de informação nas execuções maximizando a D-eficiência.

Esses desenhos de experimentos foram explorados no nível de todos os 22 casos de análise e todos os 25 participantes. Para cada desenho de experimentos, foram estabelecidas 120 configurações iniciais de experimentos, para calcular a respectiva acurácia em cada um desses experimentos. Uma vez obtidas as configurações dos hiperparâmetros com suas respectivas acurácias, avaliou-se o comportamento dos hiperparâmetros, desenhando outros 40.000 experimentos de configurações de hiperparâmetros combinados aleatoriamente, onde para cada configuração sua respectiva acurácia foi estimada treinando um modelo linear e outro modelo com interações, para então selecionar a melhor configuração do hiperparâmetros e assim calcular sua acurácia verdadeira correspondente e verificar se houve melhora em relação ao modelo base, esta verificação de melhora na acurácia foi realizada para cada participante nos diferentes casos de análise estabelecidos. O porcentagem de melhoria obtido por cada algoritmo de aprendizado de máquina pode ser visto na Tabela 4.6.

Tabela 4.6: Porcentagem de melhoria em todos os casos de análise em relação ao caso base após da aplicação de ajuste de hiperparâmetros em cada algoritmo de ML. A primeira linha mostra o porcentagem de casos melhorados e a segunda o porcentagem de casos sem melhora.

\begin{tabular}{lrrrr}
\hline & \multicolumn{4}{c}{ Experimentos (\%) } \\
& SVM & DT & LR & RF \\
\hline Melhora na acurácia & 57.3 & 19.3 & 33.3 & 36.7 \\
Sem melhora & 42.7 & 80.7 & 66.7 & 63.3 \\
\hline
\end{tabular}

Após a realização da análise acima mencionada, foram obtidas melhorias significativas na acurácia dos diferentes modelos de aprendizado de máquina, como pode ser visto na Tabela 4.7, onde são apresentadas as melhorias mais importantes obtidas na acurácia de cada algoritmo, comparando a acurácia do modelo base com a acurácia do modelo com os hiperparâmetros ajustados.

\subsection{Desempenho na seleção de métricas}

Neste trabalho, consideramos avaliar o ajuste de hiperparâmetros para o conjunto reduzido de métricas selecionadas, devido que nem sempre a mesma configuração de hiperparâmetros que existe para todas as métricas terá o mesmo efeito sobre as métricas selecionadas [DHDMN03].

Para cada um dos 22 casos de análise, uma análise da importância das métricas foi desenvolvida com a ajuda de um Desenho Ótimo de experimentos de tamanho de 120 registros, neste desenho 
Tabela 4.7: Acurácias máximas encontradas após da aplicação do ajuste de hiperparâmetros em cada algoritmo de ML. A primeira linha mostra as acurácias do modelo base, a segunda linha mostra as acurácias aplicando o ajuste de hiperparâmetros e a terceira mostra a taxa de melhoria.

\begin{tabular}{lrrrr}
\hline & \multicolumn{4}{c}{ Algoritmos de ML } \\
& SVM & DT & LR & RF \\
\hline Modelo Base & 0.61 & 0.54 & 0.65 & 0.65 \\
Modelo Hiperparâmetrizado & 0.92 & 0.87 & 0.96 & 0.87 \\
Taxa de Máxima Melhoria & 1.5 & 1.6 & 1.5 & 1.3 \\
\hline
\end{tabular}

de experimentos uma regressão linear $y(x)=\theta_{i} f_{i}(x)+\ldots+\theta_{m} f_{m}(x)+\varepsilon(x)$ foi estimada, para obter uma matriz de coeficientes $\theta$ da regressão linear, mostrando uma estimação da importância das métricas que contribuem para a acurácia. Em seguida, uma média desses coeficientes $\theta$ obtidos é estimada, para obter uma estimativa da importância das características sobre todos os casos de análise e sobre todos os 25 participantes. Antes de calcular a estimativa média dos coeficientes para cada caso de análise, foi verificada a homocedasticidade dos modelos lineares, para os quais foram realizados gráficos QQ-plot e testes de normalidade dos resíduos de Kolmogorov-Smirnov , em todos os casos de análise mostrou que os modelos lineares foram homocedásticos ( $p$-value $\geq 0.05)$. Essa heurística nos permitiu obter a Figura 4.3 onde podemos ver a média da importância das métricas ao longo de todos os casos de análise.

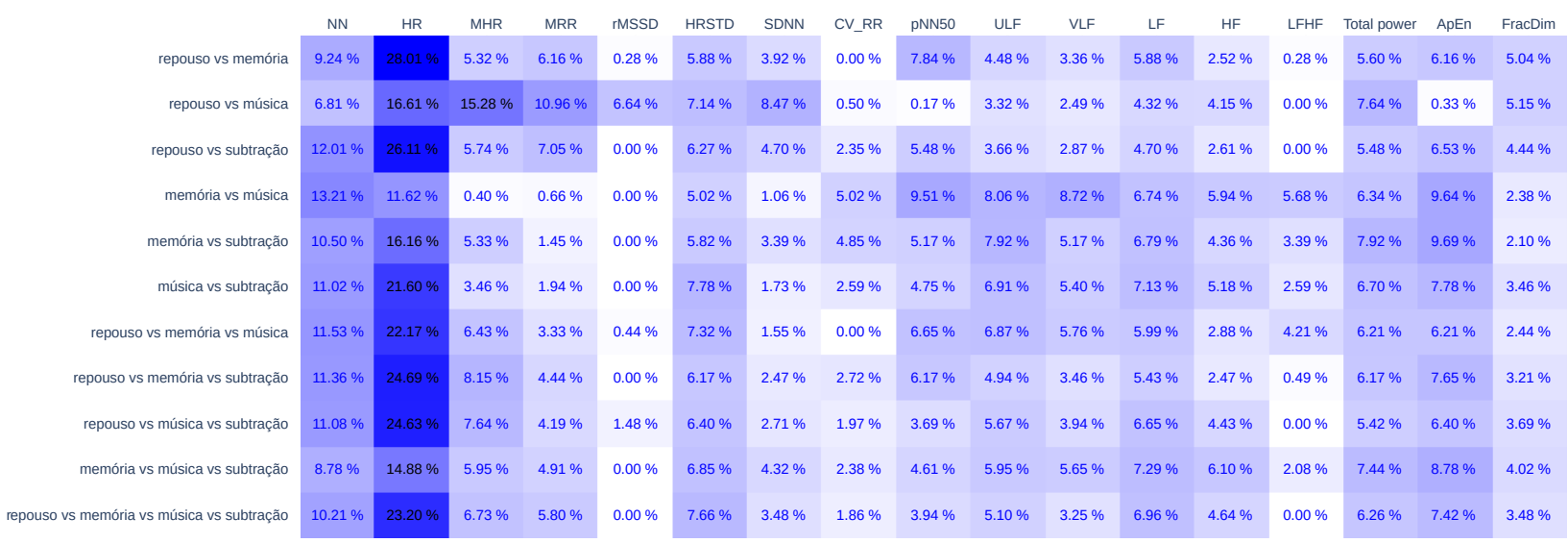

Figura 4.3: Importância das métricas nos diferentes casos de análise interdiarios

A frequência cardíaca (HR), os intervalos normalizados RR (NN) e o desvio padrão da frequência cardíaca (HRSTD) se encontraram entre as métricas mais importantes ao longo de todos os experimentos feitos. Em seguida, essas características foram selecionadas para cada caso de análise, obtendo-se melhorias significativas em cada modelo de aprendizado de máquina, a porcentagem de melhorias obtidas em todos os experimentos estão na Tabela 4.8, tendo presente que o número total de casos é 550, porque está dado ao longo dos 25 participantes e os 22 casos de análise, tal que a primeira linha mostra o porcentagem dos casos nos diferentes participantes que conseguiram melhoria na acurácia, como é caso do RF onde $40,7 \%$ dos casos avaliados conseguiram ser melhorados e na segunda linha esta o porcentagem de casos que não conseguiram melhoria em todos os algoritmos de ML.

Na Tabela 4.9 são mostradas as melhorias mais importantes obtidas na acurácia para cada algoritmo, a primeira linha mostra a acurácia no modelo base, a segunda linha mostra as melhores acurácias com as métricas selecionadas para o mesmo caso do modelo base, e a terceira linha mostra a taxa máxima de melhoria para cada algoritmo de ML, onde a melhoria mais alta foi para o DT, praticamente dobrando a acurácia do modelo base. 
Tabela 4.8: Porcentagem de melhoria em todos os casos de análise em relação ao caso base após da aplicação de seleção de métricas em cada algoritmo de ML. A primeira linha mostra o porcentagem de casos melhorados e a segunda o porcentagem de casos sem melhora.

\begin{tabular}{lrrrr}
\hline & \multicolumn{4}{c}{ Experimentos (\%) } \\
& SVM & DT & LR & RF \\
\hline Melhora na acurácia & 22.0 & 32.5 & 31.8 & 40.7 \\
Sem melhora & 78.0 & 67.5 & 68.2 & 59.3 \\
\hline
\end{tabular}

Tabela 4.9: Acurácias máximas encontradas após da aplicação de seleção de métricas em cada algoritmo de ML. A primeira linha mostra as acurácias do modelo base, a segunda linha mostra as acurácias aplicando a seleção de métricas e a terceira mostra a taxa de melhoria.

\begin{tabular}{lrrrr}
\hline & \multicolumn{4}{c}{ Algoritmos de ML } \\
& SVM & DT & LR & RF \\
\hline Modelo Base & 0.51 & 0.51 & 0.50 & 0.51 \\
Métricas Selecionadas & 0.91 & 0.99 & 0.83 & 0.89 \\
Taxa de Máxima Melhoria & 1.8 & 2.0 & 1.7 & 1.8 \\
\hline
\end{tabular}

\subsection{Desempenho de ajuste de hiperparâmetros com seleção de mé- tricas}

Com os resultados obtidos na seleção de métricas e o ajuste de hiperparâmetros confirmamos que os métodos propostos baseados em Desenhos Ótimos têm um efeito significativo na melhoria da acurácia dos modelos de aprendizado de máquina. Por isso nesta secção misturamos as potencialidades de ambos abordagens o ajuste de hiperparâmetros e a seleção de métricas. Para cada participante em cada caso da análise foi estimada o melhor conjunto de métricas com o melhor conjunto de hiperparâmetros para cada um dos distintos modelos nos diferentes algoritmos avaliados. A Tabela 4.10 mostra a porcentagem de melhorias obtidas em todos os experimentos para cada algoritmo depois de ter testado os diferentes modelos selecionando as métricas mais importantes e a melhor configuração de hiperparâmetros para cada modelo avaliado.

Tabela 4.10: Porcentagem de melhoria em todos os casos de análise em relação ao caso base após da aplicação de ajuste de hiperparâmetros com seleção de métricas em cada algoritmo de ML. A primeira linha mostra o porcentagem de casos melhorados e a segunda o porcentagem de casos sem melhora.

\begin{tabular}{lrrrr}
\hline & \multicolumn{4}{c}{ Experimentos (\%) } \\
& SVM & DT & LR & RF \\
\hline Melhora na acurácia & 58.7 & 38.0 & 52.4 & 48.5 \\
Sem Melhora & 41.3 & 62.0 & 47.6 & 51.5 \\
\hline
\end{tabular}

Na Tabela 4.11 são mostradas as melhorias mais importantes obtidas na acurácia para cada algoritmo misturando a técnica de ajuste de hiperparâmetros e seleção de métricas, a primeira linha mostra a acurácia no modelo base, a segunda linha mostra a acurácia do modelo com a mistura das técnicas e a terceira linha mostra a taxa máxima de melhoria. 
Tabela 4.11: Acurácias máximas encontradas após da aplicação do ajuste de hiperparâmetros com seleção de métricas em cada algoritmo de ML. A primeira linha mostra as acurácias do modelo base, a segunda linha mostra as acurácias aplicando o ajuste de hiperparâmetros e a terceira mostra a taxa de melhoria.

\begin{tabular}{lrrrr}
\hline & \multicolumn{4}{c}{ Algoritmos de ML } \\
& SVM & DT & LR & RF \\
\hline Modelo Base & 0.54 & 0.56 & 0.63 & 0.55 \\
Melhoria & 0.91 & 0.89 & 0.79 & 0.91 \\
Taxa de Máxima Melhoria & 1.6 & 1.7 & 1.7 & 1.7 \\
\hline
\end{tabular}

A)

- Base SVM • HP SVM • SM SVM • HP +SM SVM

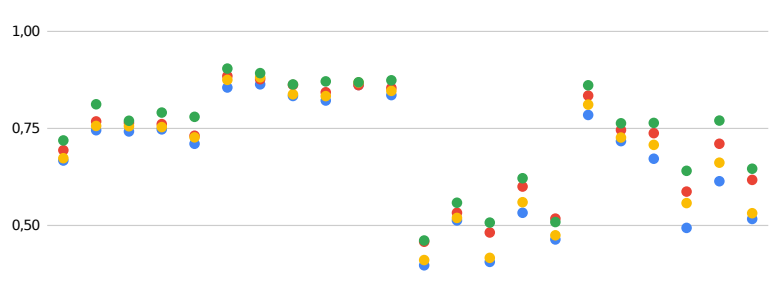

0,25

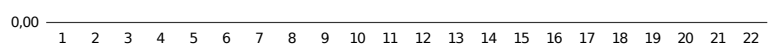

Número do Caso

C)

- Base DT • HP DT • SM DT • HP + SM DT

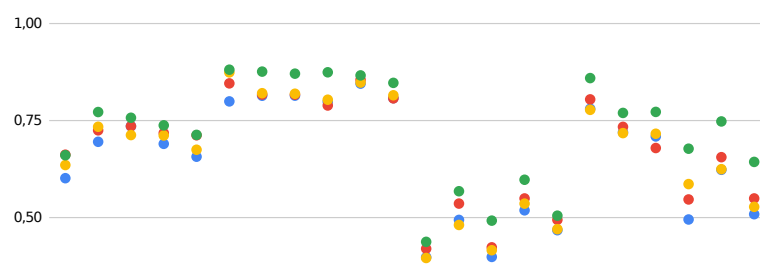

0,25

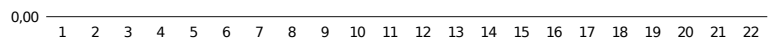

Número do Caso
B)

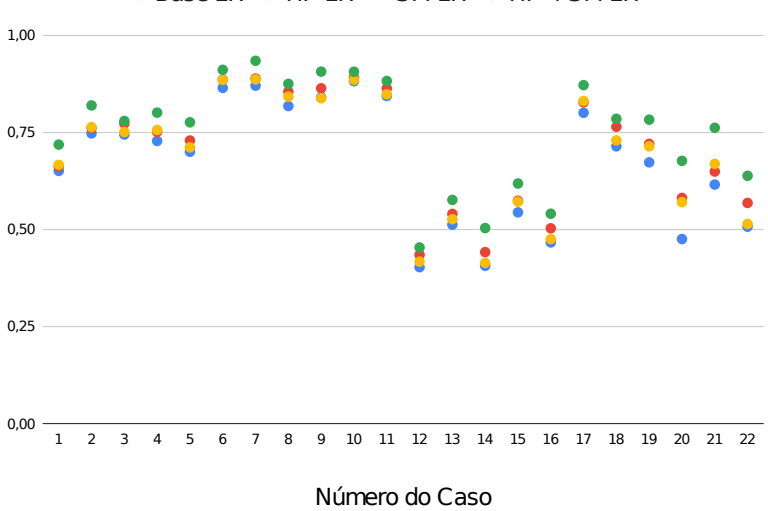

D) - Base RF • HPRF • SMRF • HP +SMRF

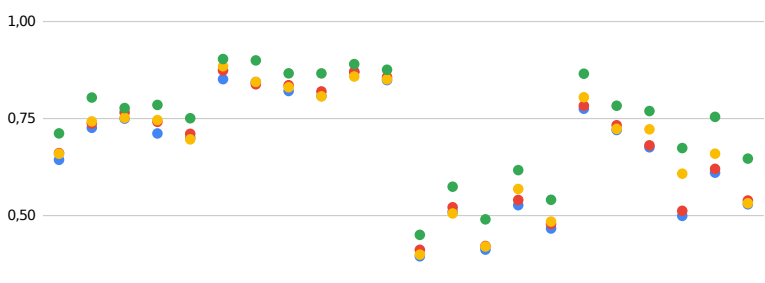

0,25

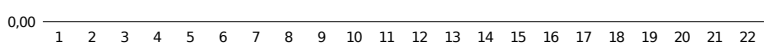

Número do Caso

Figura 4.4: Comparação da acurácia média dos modelos Base(Base), Modelos com Ajuste de Hiperparâmetros(HP), Modelos com Seleção de Métricas(SM) e Modelos juntando o HP e a SM(HP+SM) para os algoritmos de (A) SVM, (B) LR, (C) DT e (D) RF. Os casos estão ordenados de acordo a Tabela 4.1 

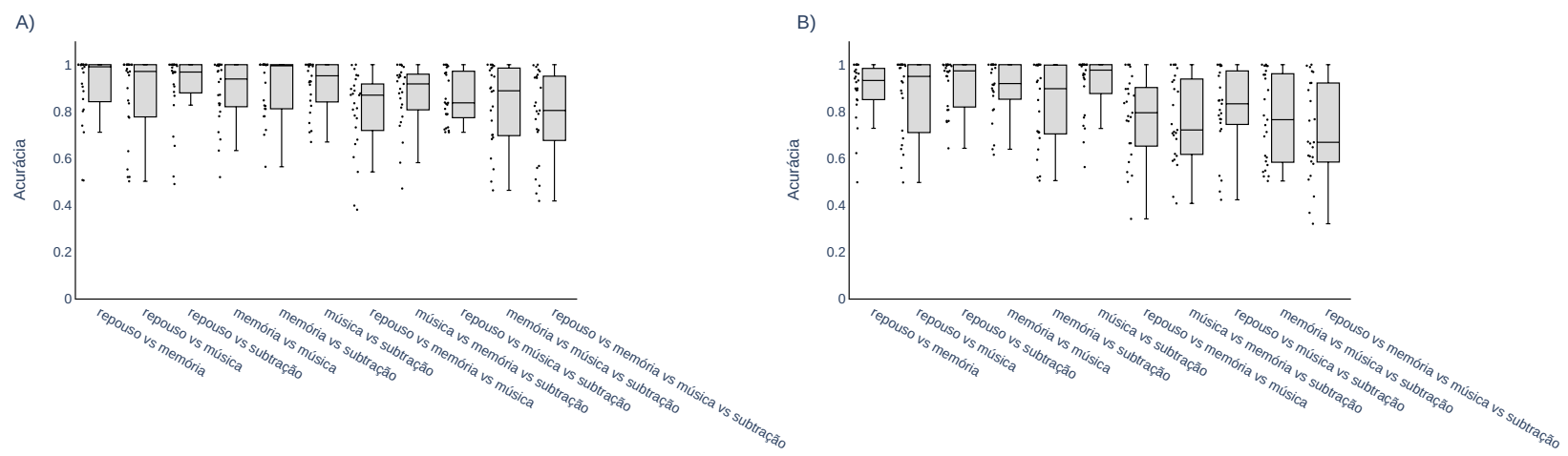

Figura 4.5: Acurácias de classificação de tarefas cognitivas intradiárias para o SVM depois de aplicar o ajuste de hiperparâmetros e a seleção de métricas. (A) Obtivemos a acurácia da classificação usando a primeira metade dos dados do dia 1 como o conjunto de treinamento e a segunda metade como o conjunto de validação. (B) Obtivemos a acurácia da classificação usando a primeira metade dos dados do dia 2 como o conjunto de treinamento e a segunda metade como o conjunto de validação.

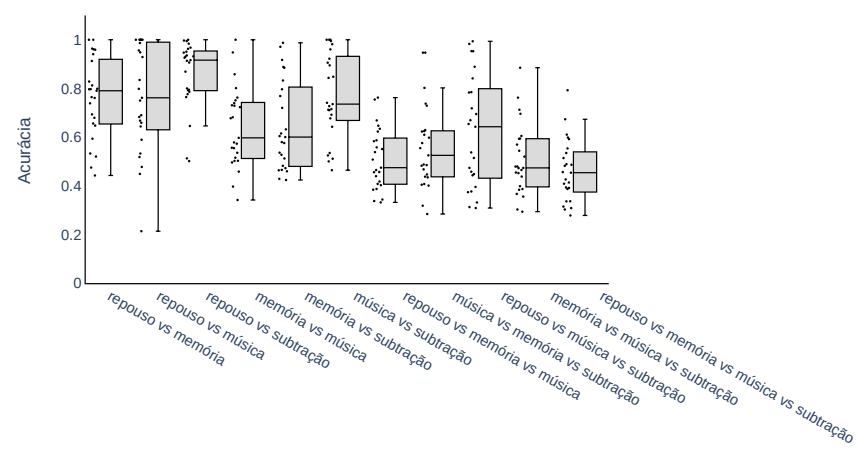

Figura 4.6: Acurácias de classificação de tarefas cognitivas interdiárias depois de aplicar o ajuste de hiperparâmetros e a seleção de métricas. Usamos os dados do dia 1 como o conjunto de treinamento e os dados do dia 2 como o conjunto de validação.

\subsection{Classificação dos participantes com base no sinal cardíaco}

Nesta seç̧ão mostramos o teste da hipótese de que o sinal cardíaco poderia ser usado como um biomarcador para discriminar o indivíduo. Para isso, organizamos os dados e os analisamos interdiário e intradiário. Para discriminar o indivíduo, utilizamos o estado de repouso e os sinais cardíacos das outras três tarefas cognitivas. No caso de treinar e classificar os indivíduos no mesmo dia, obtivemos uma acurácia de $46 \%(p=0,001)$ na tarefa de música. Também treinamos modelos de SVM usando os dados coletados no primeiro dia e validamos com os dados do segundo dia. Nesse caso, apenas a tarefa de música apresentou uma acurácia de classificação mais significativa do que esperamos ao acaso [CJ15] (acurácia de aproximadamente 20\%, $p=0,001$ ) (Figura 4.7B).

Semelhante ao que acontece na neurociência, as classificações baseadas em uma tarefa cognitiva são mais informativas do que o estado de repouso [FSD06]. 
A)

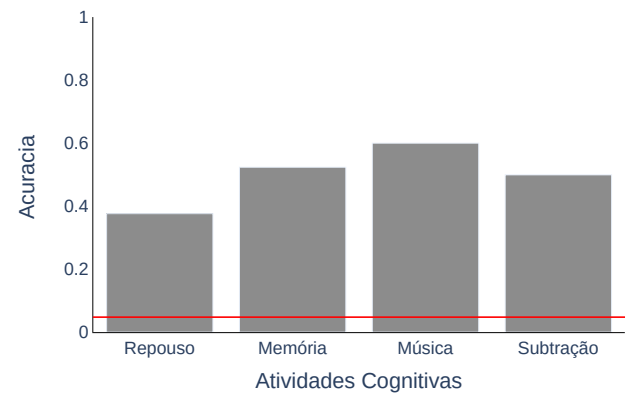

B)

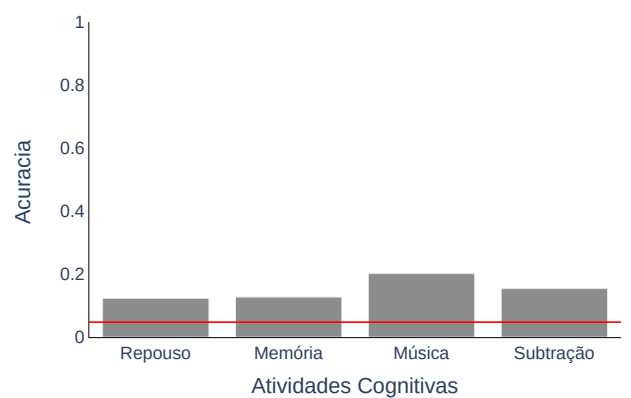

Figura 4.7: Classificação de indivíduos. (A) Obtivemos a acurácia da classificação usando a primeira metade dos dados do dia 1 como o conjunto de treinamento e a segunda metade como o conjunto de validação. (B) Obtivemos a acurácia da classificação obtida usando os dados do primeiro dia como o conjunto de treinamento e os dados do segundo dia como o conjunto de validação. 


\section{Capítulo 5}

\section{Discussão de Resultados e Conclusões}

- Para os casos de análise de classificação de atividades cognitivas no mesmo dia, em muitos participantes encontramos acurácias de 100\% (ver Figura 4.1). No entanto, no caso da classificação de atividades cognitivas em dias diferentes, a acurácia diminuiu em alguns participantes mais que outros (ver Figura 4.2). Com ajuda do ajuste de hiperparâmetros (HP) e seleção de métricas (SM) foi possível melhorar os resultados como podemos ver na Tabela 5.1. Concordamos com $\left[\mathrm{MMW}^{+}\right.$00, SvMKK02] que a variação de frequência cardíaca encontrada para os casos de análise em dias diferentes é dada pelos fatores externos que não são possíveis controlar. Mesmo assim, tentamos controlar a luminosidade, a temperatura e o horário nas diferentes sessões de coleta de dados, mas não foi possível controlar os fatores emocionais pelos quais cada pessoa pode ter passado. Também é possível observar que a acurácia de classificação diminuiu com a maior quantidade de classes, o que é esperado.

Tabela 5.1: Acurácia máxima para cada caso de análise interdiário

\begin{tabular}{l|c|c}
\hline $\begin{array}{c}\text { Número } \\
\text { de Caso }\end{array}$ & Caso de Análise & $\begin{array}{c}\text { Máxima Acurácia } \\
\text { HP+SM }\end{array}$ \\
\hline Caso 12 & 4 Classes interdiário (repouso,memória,música,subtração) & 0.71 \\
\hline Caso 13 & 3 Classes interdiário (repouso,memória,subtração) & 0.93 \\
\hline Caso 14 & 3 Classes interdiário (memória,subtração,música) & 0.85 \\
\hline Caso 15 & 3 Classes interdiário (repouso,música,subtração) & 0.93 \\
\hline Caso 16 & 3 Classes interdiário (repouso,música,memória) & 0.84 \\
\hline Caso 17 & 2 Classes interdiário (repouso, subtração) & 0.99 \\
\hline Caso 18 & 2 Classes interdiário (repouso, memória) & 0.99 \\
\hline Caso 19 & 2 Classes interdiário (repouso, música) & 0.99 \\
\hline Caso 20 & 2 Classes interdiário (memória, música) & 0.98 \\
\hline Caso 21 & 2 Classes interdiário (subtração, música) & 0.99 \\
\hline Caso 22 & 2 Classes interdiário (subtração, memória) & 0.94 \\
\hline
\end{tabular}

- A análise de importância das métricas da HRV (ver 4.3) indicou que as métricas da HRV no domínio do tempo como a frequência cardíaca e os intervalos NN junto com o pnn50, o mhr, o mrr e o sdnn foram as métricas mais importantes que contribuíram mais para uma melhor classificação das tarefas cognitivas.

- Esta pesquisa foi levantada para avaliar a possibilidade de uma alternativa técnica mais viável economicamente, devido à praticidade e portabilidade do sensor utilizado, pois em diversos trabalhos como [SRR ${ }^{+} 12$, LaC11, KL16] acostumam utilizar equipamentos mais sofisticados como o fMRI [FSD06], o EEG [NS18], o ECoG [WDS $\left.{ }^{+} 11\right]$, o ECG [SYZ ${ }^{+}$17] e o fNIRs [HKH18] para a medição das atividades psicofisiológicas porém mais caros para o mesmo fim.

- Nos casos de análise se obtiveram diferentes valores em acurácia de classificação nos parti- 
cipantes, ao distinguir essa variação de acurácia nos participantes, acreditamos que alguns participantes apresentaram certas variações psicofisiológicas em várias intensidades que alteraram seu sistema nervoso autônomo, refletindo assim nas métricas da HRV, principalmente essas variações são visíveis nos intervalos NN. Por exemplo em alguns participantes o NN diminuiu quando realizaram a tarefa de contagem regressiva. Isso nos dá uma indicação de que a tarefa de subtração requer um esforço maior do que as outras tarefas em alguns participantes. Em outras palavras, concordamos com $\left[\mathrm{BSMH}^{+} 08\right]$ que a HRV poderia ser usado para avaliar o desempenho cognitivo e o deterioro cognitivo ao longo do tempo.

- A análise de classificação destes processos cognitivos foi realizada interdiário e intradiário, permitindo assim avaliar a coerência cognitiva dos participantes ao longo dos dias, tal que acreditamos que o aprimoramento dessa abordagem deve permitir a classificação de mais estados cognitivos impulsionados pela coleta de mais dados cardíacos e pela combinação de outros sensores portáteis.

- Os resultados obtidos inicialmente com os modelos base dos diferentes algoritmos avaliados foram melhorados com as técnicas de Desenho de Experimentos que permitiu fazer o Ajuste de Hiperparâmetros (HP) e a Seleção de Métricas (SM), além disso a combinação do Ajuste de Hiperparâmetros com a Seleção de Métricas permitiu conseguir melhores acurácias em comparação de usar só um dos abordagens de forma independente, tal que permitiu ter um porcentagem del melhora em $59 \%$ dos casos para o SVM, $38 \%$ no DT, $52 \%$ no LR e $49 \%$ no RF.

- As matrizes de confusão mostram como foram confundidas as diferentes atividades cognitivas executadas entre elas para todos os participantes, a Figura 5.1 mostra a matriz de confusão para a classificação das 4 classes no mesmo dia (Caso 1) onde é possível ver que o repouso foi a atividade onde o classificador teve menor erro ao longo de todos os participantes. Na matriz da Figura 5.2 se mostra como foram confundidas as atividades cognitivas para a classificação das 4 classes em dias diferentes (Caso 12) ao longo de todos os participantes, onde a memória foi a atividade que conseguiu ser menos confundida seguida da subtração.

- No caso da identificação dos 25 participantes foi possível obter um resultado melhor que ao acaso (acurácia $=20 \%, \mathrm{p}=0,001$ ) especialmente com a tarefa de música usando os dados das sessões em dias diferentes. 
Caso 1: 4 Classes intradiário (repouso,memória,música,subtração)

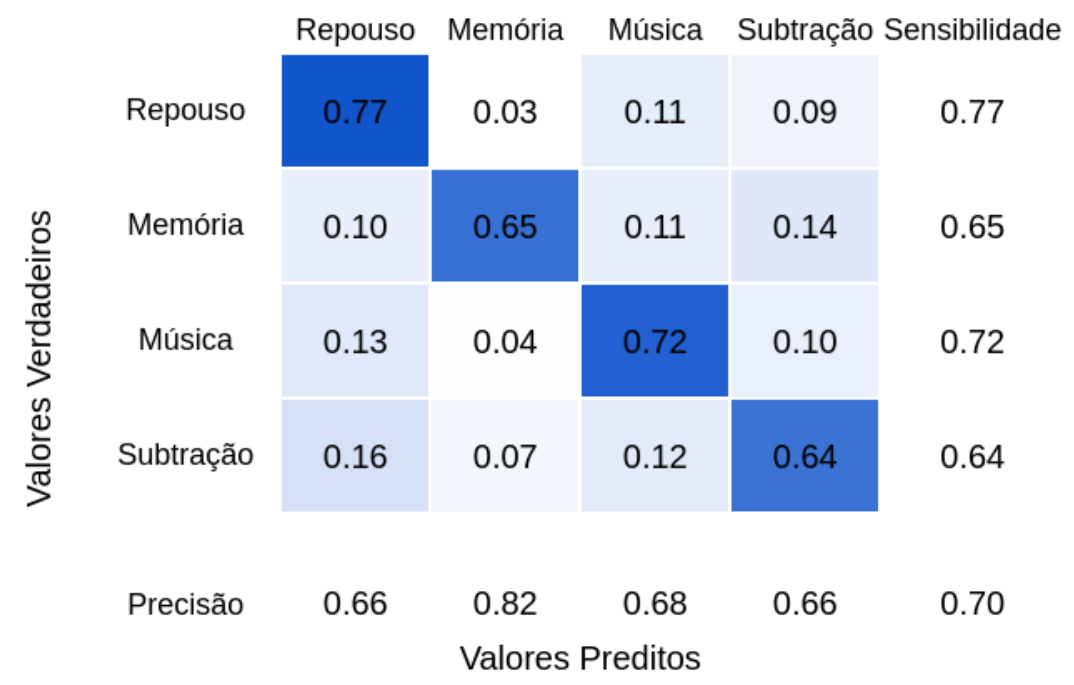

Figura 5.1: Matriz de confusão para identificação dos quatro estados cognitivos desenvolvidos no mesmo dia.

Caso 12: 4 Classes interdiário (repouso,memória,música,subtração)

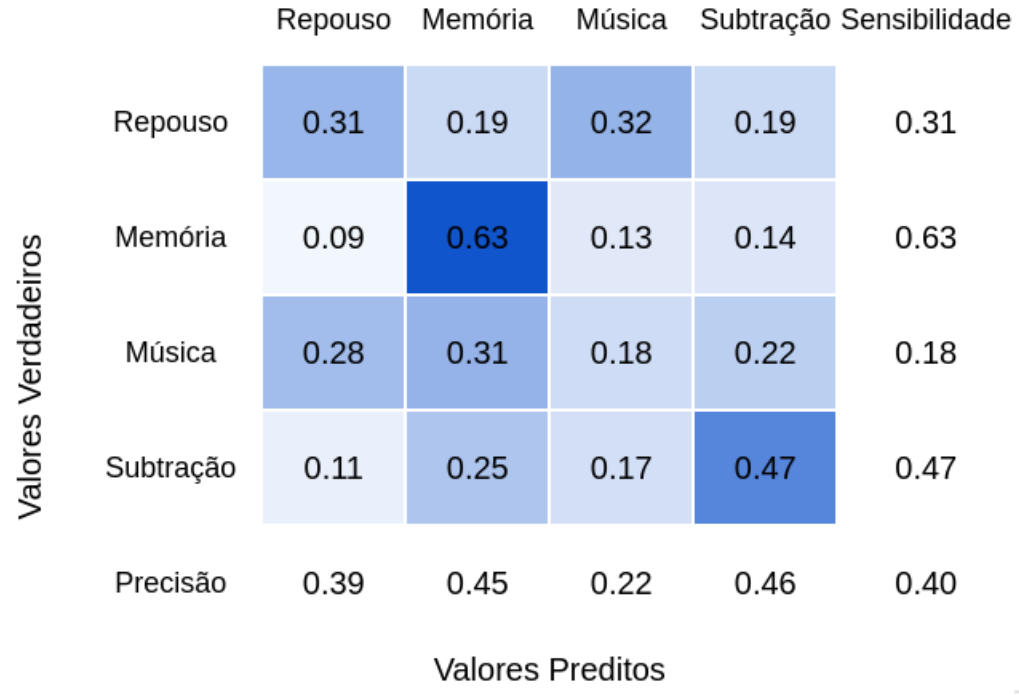

Figura 5.2: Matriz de confusão para identificação dos quatro estados cognitivos desenvolvidos em dias diferentes. 
DISCUSSÃO DE RESULTADOS E CONCLUSÕES 


\section{Apêndice A}

\section{Banco de Dados}

O dados coletados de todos os participantes podem ser encontrados y baixados no seguinte repositorio: https://github.com/caiki/HRV_DB_CognitiveStates.

\section{Apêndice B}

Comitê de Ética em Pesquisa 


\section{USP - ESCOLA DE ARTES, CIÊNCIAS E HUMANIDADES DA UNIVERSIDADE DE SÃO PAULO - EACH/USP}

\section{PARECER CONSUBSTANCIADO DO CEP}

\section{DADOS DO PROJETO DE PESQUISA}

Título da Pesquisa: Classificação da atividade baseada na variabilidade da frequência cardíaca.

Pesquisador: André Fujita

Área Temática:

Versão: 1

CAAE: 32732320.4 .0000 .5390

Instituição Proponente: Instituto de Matemática e Estatística da Universidade de São Paulo

Patrocinador Principal: UNIVERSIDADE DE SAO PAULO

\section{DADOS DO PARECER}

Número do Parecer: 4.077.287

\section{Apresentação do Projeto:}

USO DA VARIABILIDADE DA FREQUÊNCIA CARDÍACA NA CONSTRUÇÃO DE

LEITORES DE ATIVIDADE E INTERFACES HOMEM-MÁQUINA

\section{Objetivo da Pesquisa:}

a proposta deste projeto consiste em construir todo um sistema computacional para testar duas hipóteses: (1) é possível identificar a atividade executada usando apenas o sinal da VFC (equivalente ao brain reading)?; e (2) a VFC sozinha é suficiente para obter um considerável grau de controle numa interface homem-máquina?.

\section{Avaliação dos Riscos e Benefícios:}

O pesquisador informa que a pesquisa apresenta risco mínimo, e os benefícios são entender a relação do sistema nervoso autônomo com as atividades diárias.

\section{Comentários e Considerações sobre a Pesquisa:}

Pesquisa que terá importante impacto social, a partir do momento que o SNC poderá ser controlado a distância.

\section{Considerações sobre os Termos de apresentação obrigatória:}

O pesquisador apresenta todos os documentos necessários para realização da pesquisa.

\section{Recomendações:}

Recomendamos que seja detalhado o risco mínimo no TCLE.

Endereço: Av. Arlindo Béttio, ㄲo 1000

Bairro: Ermelino Matarazzo

CEP: $03.828-000$ 


\section{USP - ESCOLA DE ARTES, CIÊNCIAS E HUMANIDADES DA UNIVERSIDADE DE SÃO PAULO - EACH/USP}

Continuação do Parecer: 4.077.287

Conclusões ou Pendências e Lista de Inadequações:

Não há

Considerações Finais a critério do CEP:

Este parecer foi elaborado baseado nos documentos abaixo relacionados:

\begin{tabular}{|c|c|c|c|c|}
\hline Tipo Documento & Arquivo & Postagem & Autor & Situação \\
\hline $\begin{array}{l}\text { Informações Básicas } \\
\text { do Projeto }\end{array}$ & $\begin{array}{l}\text { PB_INFORMAÇÕES_BÁSICAS_DO_P } \\
\text { ROJETO 1325088.pdf }\end{array}$ & $\begin{array}{c}28 / 05 / 2020 \\
13: 29: 57 \\
\end{array}$ & & Aceito \\
\hline $\begin{array}{l}\text { TCLE / Termos de } \\
\text { Assentimento / } \\
\text { Justificativa de } \\
\text { Ausência }\end{array}$ & TCLE.pdf & $\begin{array}{l}28 / 05 / 2020 \\
13: 28: 12\end{array}$ & André Fujita & Aceito \\
\hline Outros & PolarH10.jpg & $\begin{array}{c}28 / 05 / 2020 \\
13: 26: 20 \\
\end{array}$ & André Fujita & Aceito \\
\hline \begin{tabular}{|l} 
Declaração de \\
Instituição e \\
Infraestrutura \\
\end{tabular} & termo_consentimento.pdf & $\begin{array}{l}\text { 28/05/2020 } \\
13: 19: 56\end{array}$ & André Fujita & Aceito \\
\hline Outros & CARTA_PROTOCOLO_PESQUISA.pdf & $\begin{array}{c}28 / 05 / 2020 \\
13: 19: 06 \\
\end{array}$ & André Fujita & Aceito \\
\hline \begin{tabular}{|l|} 
Projeto Detalhado / \\
Brochura \\
Investigador \\
\end{tabular} & ProjetoPRP.pdf & $\begin{array}{c}\text { 07/05/2020 } \\
16: 43: 39\end{array}$ & André Fujita & Aceito \\
\hline Folha de Rosto & folhaderostosigned.pdf & $\begin{array}{c}07 / 05 / 2020 \\
15: 30: 46 \\
\end{array}$ & André Fujita & Aceito \\
\hline
\end{tabular}

\section{Situação do Parecer:}

Aprovado

Necessita Apreciação da CONEP:

Não

SAO PAULO, 09 de Junho de 2020

Assinado por:

Beatriz Aparecida Ozello Gutierrez

(Coordenador(a))

Endereço: Av. Arlindo Béttio, no 1000

Bairro: Ermelino Matarazzo

CEP: $\quad 03.828-000$

UF: SP

Município: SAO PAULO

Telefone:

(11)3091-1046

E-mail: cep-each@usp.br 


\section{INSTITUTO DE MATEMÁTICA E ESTATÍSTICA \\ UNIVERSIDADE DE SÃO PAULO}

\section{TERMO DE CONSENTIMENTO LIVRE E ESCLARECIDO}

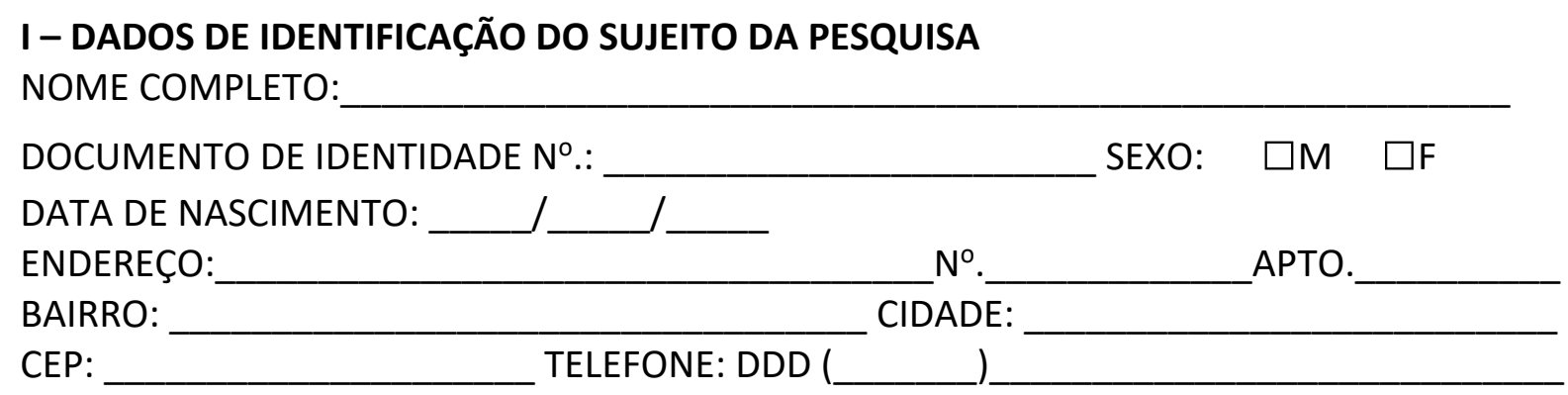

\section{II - DADOS SOBRE A PESQUISA CIENTÍFICA}

1. TÍTULO DO PROJETO DE PESQUISA: Classificação da atividade baseada na variabilidade da frequência cardíaca.

2. PESQUISADOR RESPONSÁVEL: André Fujita

3. CARGO / FUNÇÃO: Professor Associado

4. AVALIAÇÃO DO RISCO DA PESQUISA:

囚RISCO MÍNIMO $\square$ RISCO MÉDIO

(probabilidade de que o indivíduo sofra algum dano como consequência imediata ou tardia do estudo)

5. DURAÇÃO DA PESQUISA

Cada sujeito terá 2 (duas) sessões de coletas de dados com intervalo mínimo de 1 (uma) semana entre elas. Desse modo, cada sessão de coleta será agendada previamente com o sujeito respeitando sua disponibilidade. Após conclusão das duas sessões o sujeito terá terminada sua participação no estudo.

\section{III - EXPLICAÇÕES DO PESQUISADOR AO INDIVÍDUO SOBRE A PESQUISA, CONSIGNANDO:}

A frequência cardíaca de 30 indivíduos, todos do sexo masculino serão monitorados ao longo do tempo. Cada sujeito terá 2 (duas) sessões de coletas de dados com intervalo mínimo de uma semana entre elas. Todos os indivíduos serão submetidos a cinco tarefas com duração de 10 minutos cada: (1) estado de repouso, (2) tarefa de memória, (3) tarefa de música, (4) tarefa de subtração, (5) tarefa de imaginação espacial. Sempre o estado de repouso será completado primeiro, e a ordem das demais tarefas serão bem balanceadas entre os 30 indivíduos.

1. ESTADO DE REPOUSO: os indivíduos serão instruídos a não pensar em nada de forma focada.

2. TAREFA DE MEMÓRIA: os indivíduos serão instruídos a lembrar dos eventos que ocorreram desde o dia anterior até o momento do experimento. 
3. TAREFA DE MÚSICA: os indivíduos serão instruídos a cantarem suas músicas favoritas mentalmente.

4. TAREFA DE SUBTRAÇÃO: os indivíduos serão instruídos a contarem de 5.000 de sete em sete.

5. TAREFA DE IMAGINAÇÃO ESPACIAL: os indivíduos serão instruídos a imaginar andando pela sala onde estão.

Para todas as tarefas de 1 a 5 , os indivíduos serão instruídos a se sentarem e manterem seus olhos fechados durante todo o experimento.

Destacamos ainda que os experimentos serão realizados de forma não-invasiva (uso de uma cinta peitoral Polar). Portanto, não há riscos a sua integridade física.

A sua participação no estudo contribuirá para o avanço do conhecimento na área de interfaces homem-máquina.

\section{IV - ESCLARECIMENTOS DADOS PELO PESQUISADOR SOBRE GARANTIAS DO SUJEITO DA PESQUISA:}

Ao participar desta pesquisa você tem acesso, a qualquer tempo, as informações sobre procedimentos, riscos e benefícios relacionados a pesquisa, inclusive para dirimir eventuais dúvidas. Também tem liberdade de retirar seu consentimento a qualquer momento e de deixar de participar do estudo, sem que isto traga prejuízo a continuidade da assistência.

Todos os dados coletados e declarados por você são salvaguardados de confidencialidade, sigilo e privacidade.

A Universidade de São Paulo coloca a disponibilidade a assistência no Hospital Universitário (HU) para eventuais danos a saúde, decorrentes da pesquisa.

\section{V - INFORMAÇÕES DE NOMES, ENDEREÇOS E TELEFONES DOS RESPONSÁVEIS PELO ACOMPANHAMENTO DA PESQUISA, PARA CONTATO EM CASO DE INTERCORRÊNCIAS CLÍNICAS E REAÇÕES ADVERSAS.}

Prof. Dr. André Fujita

Rua do Matão, 1010 - Cidade Universitária - 05508-090

Tel.: (11) 30915177

\section{VI - CONSENTIMENTO PÓS-ESCLARECIDO}

Declaro que, após convenientemente esclarecido pelo pesquisador e ter entendido o que me foi explicado, consinto em participar do presente Projeto de Pesquisa. Também declaro estar ciente de que tanto os resultados da pesquisa como os dados (que não permitam minha identificação) poderão ser publicados.

São Paulo, de de 20 


\section{Referências Bibliográficas}

[20007] Design of Experiments. Em Analytic Methods for Design Practice, páginas 309391. Springer London, London, 2007. 18

[AD89] A. C. Atkinson e A. N. Donev. The construction of exact d-optimum experimental designs with application to blocking response surface designs. Biometrika, 76(3):515-526, 9 1989. 20, 35

[ALAAANAT] Hilal Al-Libawy, Ali Al-Ataby, Waleed Al-Nuaimy e Majid A Al-Taee. HRV-Based Operator Fatigue Analysis and Classification Using Wearable Sensors. Relatório técnico. 8

[ALAAANAT16] Hilal Al-Libawy, Ali Al-Ataby, Waleed Al-Nuaimy e Majid A. Al-Taee. HRVbased operator fatigue analysis and classification using wearable sensors. Em 13th International Multi-Conference on Systems, Signals and Devices, SSD 2016, páginas 268-273. Institute of Electrical and Electronics Engineers Inc., 52016. 12

[Alt17] Miguel Alfonso Altuve. Variabilidad de la frecuencia cardíaca : Técnicas temporales, frecuenciales y no lineales, 2017. 2, 5, 11

[APK07] Paula Alhola e Päivi Polo-Kantola. Sleep deprivation: Impact on cognitive performance. Neuropsychiatric disease and treatment, 2007. 6

[ASHM00] Burak Acar, Irina Savelieva, Harry Hemingway e Marek Malik. Automatic ectopic beat elimination in short-term heart rate variability measurement. Computer Methods and Programs in Biomedicine, 63(2):123-131, 10 2000. 26

[ASM08] Babak Mohammadzadeh Asl, Seyed Kamaledin Setarehdan e Maryam Mohebbi. Support vector machine-based arrhythmia classification using reduced features of heart rate variability signal. Artificial Intelligence in Medicine, 44(1):51-64, 9 2008. 12

[ASS ${ }^{+}$16] A. Altmann, M. S. Schröter, V. I. Spoormaker, S. A. Kiem, D. Jordan, R. Ilg, E. T. Bullmore, M. D. Greicius, M. Czisch e P. G. Sämann. Validation of nonREM sleep stage decoding from resting state fMRI using linear support vector machines. NeuroImage, 125:544-555, 1 2016. 7

$\left[\mathrm{BAJ}^{+} 19\right]$ Soheil Borhani, Reza Abiri, Yang Jiang, Taylor Berger e Xiaopeng Zhao. Brain connectivity evaluation during selective attention using EEG-based braincomputer interface. Brain-Computer Interfaces, 6(1-2):25-35, 2019. 7

[BBBK11] James Bergstra, Rémi Bardenet, Yoshua Bengio e Balázs Kégl. Algorithms for Hyper-Parameter Optimization. Relatório técnico, 2011. 17

[BCC12] James Bergstra, James Bergstra@umontreal Ca e Yoshua Bengio@umontreal Ca. Random Search for Hyper-Parameter Optimization Yoshua Bengio. Relatório Técnico 10, 2012. 17 
[BDDS05] Christian F. Beckmann, Marilena DeLuca, Joseph T. Devlin e Stephen M. Smith. Investigations into resting-state connectivity using independent component analysis. Philosophical Transactions of the Royal Society B: Biological Sciences, 360(1457):1001-1013, 2005. 1

[BFS ${ }^{+}$92] J T Bigger, J L Fleiss, R C Steinman, L M Rolnitzky, R E Kleiger e J N Rottman. Frequency domain measures of heart period variability and mortality after myocardial infarction. Circulation, 85(1):164-171, 1 1992. 11

$\left[\mathrm{BKK}^{+} 17\right]$ Rohan K. Bodapati, Jorge R. Kizer, Willem J. Kop, Hooman Kamel e Phyllis K. Stein. Addition of 24-Hour Heart Rate Variability Parameters to the Cardiovascular Health Study Stroke Risk Score and Prediction of Incident Stroke: The Cardiovascular Health Study. Journal of the American Heart Association, 6(7), 7 2017. 9

[BMMFT18] Neeltje J. Boogert, Joah R. Madden, Julie Morand-Ferron e Alex Thornton. Measuring and understanding individual differences in cognition, 9 2018. 6

[BP94] John J. Borkowski e Friedrich Pukelsheim. Optimal Design of Experiments, volume 36. SIAM, 1994. 19, 20

$\left[\mathrm{BPJ}^{+}\right.$06] Pierre Bellec, Vincent Perlbarg, Saâd Jbabdi, Mélanie Pélégrini-Issac, Jean Luc Anton, Julien Doyon e Habib Benali. Identification of large-scale networks in the brain using fMRI. NeuroImage, 29(4):1231-1243, 2 2006. 1

[Bre99] Leo Breiman. Random Forests - Random Features. Technical Report 567. Relatório técnico, University of California, Berkeley, 1999. 15

[Bru20] Pedro Bruel. ExperimentalDesign.jl, 2020. 35

$\left[\mathrm{BSM}^{+} 07\right]$ Annie Britton, Martin Shipley, Marek Malik, Katerina Hnatkova, Harry Hemingway e Mlichael Marmot. Changes in Heart Rate and Heart Rate Variability Over Time in Middle-Aged Men and Women in the General Population (from the Whitehall II Cohort Study). The American Journal of Cardiology, 100(3):524527, 8 2007. 12

$\left[\mathrm{BSMH}^{+} 08\right]$ Annie Britton, Archana Singh-Manoux, Katerina Hnatkova, Marek Malik, Michael G. Marmot e Martin Shipley. The Association between Heart Rate Variability and Cognitive Impairment in Middle-Aged Men and Women. Neuroepidemiology, 31(2):115-121, 9 2008. 42

[BZYHH95] Bharat Biswal, F. Zerrin Yetkin, Victor M. Haughton e James S. Hyde. Functional connectivity in the motor cortex of resting human brain using echo-planar mri. Magnetic Resonance in Medicine, 34(4):537-541, 10 1995. 1

[CDM15] Marc Claesen e Bart De Moor. Hyperparameter Search in Machine Learning. 2 2015. 17

$\left[\mathrm{CFS}^{+}\right.$07] Robert M. Carney, Kenneth E. Freedland, Phyllis K. Stein, Gregory E. Miller, Brian Steinmeyer, Michael W. Rich e Stephen P. Duntley. Heart rate variability and markers of inflammation and coagulation in depressed patients with coronary heart disease. Journal of Psychosomatic Research, 62(4):463-467, 4 2007. 11

[Cha21] Robin Champseix. hrv-analysis · PyPI, 2021. 27

[CJ15] Etienne Combrisson e Karim Jerbi. Exceeding chance level by chance: The caveat of theoretical chance levels in brain signal classification and statistical assessment of decoding accuracy. Journal of Neuroscience Methods, 250:126-136, 7 2015. 39 
$\left[\mathrm{CKK}^{+} 17\right]$ Kwang-Ho Choi, Junbeom Kim, O. Sang Kwon, Min Ji Kim, Yeon Hee Ryu e JiEun Park. Is heart rate variability (HRV) an adequate tool for evaluating human emotions? - A focus on the use of the International Affective Picture System (IAPS). Psychiatry Research, 251:192-196, 5 2017. 10, 12

[Cli02] Gari D Clifford. Signal Processing Methods for Heart Rate Variability. Tese de Doutorado, University of Oxford, Oxford, 2002. 27

[CN14] Daniel E. Callan e Eiichi Naito. Neural Processes Distinguishing Elite from Expert and Novice Athletes. Cognitive and Behavioral Neurology, 27(4):183-188, 12 2014. 7

[Cra03] A. D. Craig. Interoception: The sense of the physiological condition of the body, 8 2003. 1

[CST00] Nello Cristianini e John Shawe-Taylor. Support Vector Machines. Em An Introduction to Support Vector Machines and Other Kernel-based Learning Methods, páginas 93-124. Cambridge University Press, 3 2000. 13

[CYBB17] Abdullah Caliskan, Mehmet Emin Yuksel, Hasan Badem e Alper Basturk. A deep neural network classifier for decoding human brain activity based on magnetoencephalography. Elektronika ir Elektrotechnika, 23(2):63-67, 4 2017. 7

[DC04] Michael E. Dewey e Chih Mei Chen. Neurosis and mortality in persons aged 65 and over living in the community: A systematic review of the literature. International Journal of Geriatric Psychiatry, 19(6):554-557, 2004. 6

[DHDMN03] Walter Daelemans, Véronique Hoste, Fien De Meulder e Bart Naudts. Combined optimization of feature selection and algorithm parameters in machine learning of language. Em Lecture Notes in Artificial Intelligence (Subseries of Lecture Notes in Computer Science), volume 2837, páginas 84-95. Springer Verlag, 2003. 35

[DOM02] Stephan Dreiseitl e Lucila Ohno-Machado. Logistic regression and artificial neural network classification models: A methodology review. Journal of Biomedical Informatics, 35(5-6):352-359, 10 2002. 14

[DPZ13] Holger Dette, Andrey Pepelyshev e Anatoly Zhigljavsky. Optimal design for linear models with correlated observations. Annals of Statistics, 41(1):143-176, 32013. 20

$\left[\mathrm{DRB}^{+} 06\right]$ J. S. Damoiseaux, S. A.R.B. Rombouts, F. Barkhof, P. Scheltens, C. J. Stam, S. M. Smith e C. F. Beckmann. Consistent resting-state networks across healthy subjects. Proceedings of the National Academy of Sciences of the United States of America, 103(37):13848-13853, 9 2006. 1

[DT16] Adina E. Draghici e J. Andrew Taylor. The physiological basis and measurement of heart rate variability in humans, 1 2016. xiii, 9

[DYL19] F. John Dian, Amirhossein Yousefi e Sungjoon Lim. A practical study on Bluetooth Low Energy (BLE) throughput. Em 2018 IEEE 9th Annual Information Technology, Electronics and Mobile Communication Conference, IEMCON 2018, páginas 768-771. Institute of Electrical and Electronics Engineers Inc., 1 2019. 8

[EDSC07] Michael W Eysenck, Nazanin Derakshan, Rita Santos e Manuel G Calvo. Anxiety and cognitive performance: attentional control theory. Emotion, 7(2):336, 2007. 
$\left[\mathrm{EPB}^{+} 11\right]$ Andrew J. Elliot, Vincent Payen, Jeanick Brisswalter, Francois Cury e Julian F. Thayer. A subtle threat cue, heart rate variability, and cognitive performance. Psychophysiology, 48(10):1340-1345, 10 2011. 7

[Fed13] Valerii Vadimovich Fedorov. Theory of optimal experiments. Elsevier, 2013. 20

[FFC19] Giuseppe Forte, Francesca Favieri e Maria Casagrande. Heart Rate Variability and Cognitive Function: A Systematic Review. Frontiers in Neuroscience, 13(JUL):710, 7 2019. 2

[FH19] Matthias Feurer e Frank Hutter. Hyperparameter Optimization. Em Automated Machine Learning, chapter 1, páginas 3-33. Springer, Cham, 2019. 17

[FLZH19] Pedro Ferreira, Duc C. Le e Nur Zincir-Heywood. Exploring Feature Normalization and Temporal Information for Machine Learning Based Insider Threat Detection. Em 15th International Conference on Network and Service Management, CNSM 2019. Institute of Electrical and Electronics Engineers Inc., 10 2019. 31

[FPea11] A. Gramfort F. Pedregosa, G. Varoquaux e et al. Scikit-learn: Machine Learning in Python. Journal of Machine Learning Research, 12(85):2825-2830, 2011. 17, 33

[FSD06] Yong Fan, Dinggang Shen e Christos Davatzikos. Detecting cognitive states from fMRI images by machine learning and multivariate classification. Em Proceedings of the IEEE Computer Society Conference on Computer Vision and Pattern Recognition, volume 2006, 2006. 39, 41

[GDN16] David Giles, Nick Draper e William Neil. Validity of the Polar V800 heart rate monitor to measure RR intervals at rest. European Journal of Applied Physiology, 116(3):563-571, 3 2016. 8, 27

[GKRM03] Michael D. Greicius, Ben Krasnow, Allan L. Reiss e Vinod Menon. Functional connectivity in the resting brain: A network analysis of the default mode hypothesis. Proceedings of the National Academy of Sciences of the United States of America, 100(1):253-258, 1 2003. 1

[GQVMCJ07] Felipe García Quiroz, Adriana Villa Moreno e Paula Castaño Jaramillo. Interfaces neuronales y sistemas máquina cerebro: Fundamentos y aplicaciones. Revisión. $\underline{\text { Revista Ingeniería Biomédica, 1:14 - 22, 2007. } 7}$

[GRP00] C. Guger, H. Ramoser e G. Pfurtscheller. Real-time EEG analysis with subjectspecific spatial patterns for a brain-computer interface (BCI). IEEE Transactions on Rehabilitation Engineering, 8(4):447-456, 2000. 7

[GT07] Paul Grossman e Edwin W. Taylor. Toward understanding respiratory sinus arrhythmia: Relations to cardiac vagal tone, evolution and biobehavioral functions. Biological Psychology, 74(2):263-285, 2 2007. 11

[HKH18] Keum Shik Hong, M. Jawad Khan e Melissa J. Hong. Feature Extraction and Classification Methods for Hybrid fNIRS-EEG Brain-Computer Interfaces, 6 2018. 41

[HL02] Chih Wei Hsu e Chih Jen Lin. A comparison of methods for multiclass support vector machines. IEEE Transactions on Neural Networks, 13(2):415-425, 32002. 13

[HPS ${ }^{+}$02] Michelle Hampson, Bradley S. Peterson, Pawel Skudlarski, James C. Gatenby e John C. Gore. Detection of functional connectivity using temporal correlations in MR images. Human Brain Mapping, 15(4):247-262, 2002. 1 
[HR06] John Dylan Haynes e Geraint Rees. Decoding mental states from brain activity in humans, 7 2006. 1, 7

[Jul06] Claude Julien. The enigma of Mayer waves: Facts and models, 4 2006. 2

[KF95] M V Kamath e E L Fallen. Correction of the Heart Rate Variability Signal for Ectopics and Missing Beats, In: Malik M., Camm AJ (eds.): Heart Rate Variability. Armonk, NY Futura Pub. Co. Inc, 1995. 26

[KFP91] Daniel T. Kaplan, Mark I. Furman e Steven M. Pincus. Techniques for analyzing complexity in heart rate and beat-to-beat blood pressure signals. Em Computers in Cardiology, páginas 243-246. Publ by IEEE, 5 1991. 12

[KHA07] Kathi J. Kemper, Craig Hamilton e Mike Atkinson. Heart rate variability: Impact of differences in outlier identification and management strategies on common measures in three clinical populations. Pediatric Research, 62(3):337-342, 92007. 26

[KHH14] M. Jawad Khan, Melissa Jiyoun Hong e Keum-Shik Hong. Decoding of four movement directions using hybrid NIRS-EEG brain-computer interface. Frontiers in Human Neuroscience, 8(1 APR):244, 4 2014. 7

[KK06] S B Kotsiantis e D Kanellopoulos. Data preprocessing for supervised leaning. International Journal of ..., 1(2):1-7, 2006. 26

$\left[\mathrm{KKJ}^{+}\right.$03] Vesa Kiviniemi, Juha Heikki Kantola, Jukka Jauhiainen, Aapo Hyvärinen e Osmo Tervonen. Independent component analysis of nondeterministic fMRI signal sources. NeuroImage, 19(2):253-260, 6 2003. 1

[KL16] Philip A. Kragel e Kevin S. LaBar. Decoding the Nature of Emotion in the Brain, 6 2016. 7, 41

[KMN09] A. Kampouraki, G. Manis e C. Nikou. Heartbeat Time Series Classification With Support Vector Machines. IEEE Transactions on Information Technology in Biomedicine, 13(4):512-518, 7 2009. 12

$\left[\mathrm{KQG}^{+} 10\right]$ Andrew H. Kemp, Daniel S. Quintana, Marcus A. Gray, Kim L. Felmingham, Kerri Brown e Justine M. Gatt. Impact of Depression and Antidepressant Treatment on Heart Rate Variability: A Review and Meta-Analysis. Biological Psychiatry, 67(11):1067-1074, 6 2010. 12

[KSB05] Robert E. Kleiger, Phyllis K. Stein e J. Thomas Bigger. Heart Rate Variability: Measurement and Clinical Utility. Annals of Noninvasive Electrocardiology, 10(1):88-101, 1 2005. 11

[KSR ${ }^{+}$09] Vesa Kiviniemi, Tuomo Starck, Jukka Remes, Xiangyu Long, Juha Nikkinen, Marianne Haapea, Juha Veijola, Irma Moilanen, Matti Isohanni, Yu-Feng Zang e Osmo Tervonen. Functional segmentation of the brain cortex using high model order group PICA. Human Brain Mapping, 30(12):3865-3886, 12 2009. 1

[LaC11] Stephen M. LaConte. Decoding fMRI brain states in real-time. NeuroImage, 56(2):440-454, 5 2011. 41

$\left[\mathrm{LBS}^{+}\right.$08] Rachel Lampert, J. Douglas Bremner, Shaoyong Su, Andrew Miller, Forrester Lee, Faiz Cheema, Jack Goldberg e Viola Vaccarino. Decreased heart rate variability is associated with higher levels of inflammation in middle-aged men. American Heart Journal, 156(4):1-759, 10 2008. 11 
$\left[\mathrm{LHW}^{+}\right]$Thomas Navin Lal, Thilo Hinterberger, Guido Widman, Michael Schröder, Jeremy Hill, Wolfgang Rosenstiel, Christian E Elger, Bernhard Schölkopf e Niels Birbaumer. Methods Towards Invasive Human Brain Computer Interfaces. Relatório técnico. 7

$\left[\mathrm{LLY}^{+}\right.$06] Ruiming Liu, Erqi Liu, Jie Yang, Ming Li e Fanglin Wang. Optimizing the Hyperparameters for SVM by Combining Evolution Strategies with a Grid Search. Em Intelligent Control and Automation, páginas 712-721. Springer Berlin Heidelberg, 102006.17

[LR07] Víctor Ignacio López e Rogelio Ramos. Una introducción a los diseños óptimos.

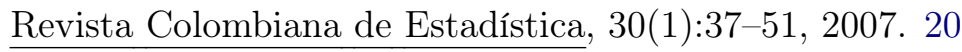

[LTMC08] Xia Lin, Christopher R Tench, Paul S Morgan e Cris S Constantinescu. Use of combined conventional and quantitative MRI to quantify pathology related to cognitive impairment in multiple sclerosis. Journal of Neurology, Neurosurgery \& Psychiatry, 79(4):437-441, 2008. 6

[LY13] Guo Cheng Liu e Hong Yang Yu. Design and implementation of a Bluetooth 4.0-based heart rate monitor system on iOS platform. Em 2013 International Conference on Communications, Circuits and Systems, ICCCAS 2013, volume 2, páginas 112-115. IEEE Computer Society, 2013. 23

[LZOC] Ching-Liang Lu, Xiaoping Zou, William C. Orr e J. D. Z. Chen. Postprandial Changes of Sympathovagal Balance Measured by Heart Rate Variability. Digestive Diseases and Sciences, 44(4):857-861. 12

[Mal96] Marek Malik. Heart rate variability: Standards of measurement, physiological interpretation, and clinical use. Circulation, 93:1043-1065, 1996. 11

$\left[\mathrm{MBC}^{+} 96\right]$ M. Malik, J. T. Bigger, A. J. Camm, R. E. Kleiger, A. Malliani, A. J. Moss e P. J. Schwartz. Heart rate variability: Standards of measurement, physiological interpretation, and clinical use. European Heart Journal, 17(3):354-381, 31996. $9,10,11$

$\left[\mathrm{MCP}^{+} 10\right]$ Donatella Marazziti, Giorgio Consoli, Michela Picchetti, Marina Carlini e Luca Faravelli. Cognitive impairment in major depression. European journal of pharmacology, 626(1):83-86, 2010. 6

[MF19] Naveen Masood e Humera Farooq. Investigating EEG Patterns for Dual-Stimuli Induced Human Fear Emotional State. Sensors, 19(3):522, 1 2019. 7

$\left[\mathrm{MHN}^{+} 04\right]$ Tom M. Mitchell, Rebecca Hutchinson, Radu S. Niculescu, Francisco Pereira, Xuerui Wang, Marcel Just e Sharlene Newman. Learning to decode cognitive states from brain images. Machine Learning, 57(1-2 SPEC. ISS.):145-175, 10 2004. 1

[Mik97] Mario Mikulincer. Adult attachment style and information processing: Individual differences in curiosity and cognitive closure. Journal of personality and social $\underline{\text { psychology, } 72(5): 1217,1997.6}$

$\left[\mathrm{MMJ}^{+} 11\right]$ Nathan T. Mowery, John A. Morris, Judith M. Jenkins, Asli Ozdas e Patrick R. Norris. Core temperature variation is associated with heart rate variability independent of cardiac index: A study of 278 trauma patients. Journal of Critical Care, 26(5):9-534, 2011. 12 
$\left[\mathrm{MMW}^{+} 00\right] \mathrm{M}$ M Massin, K Maeyns, N Withofs, F Ravet e P Gérard. Circadian rhythm of heart rate and heart rate variability. Archives of disease in childhood, 83(2):17982,8 2000. 12, 41

[Mon92] Douglas C. Montgomery. The Use of Statistical Process Control and Design of Experiments in Product and Process Improvement. IIE Transactions (Institute of Industrial Engineers), 24(5):4-17, 1992. 18

[MPB05] Weng-Kee Wong Martijn P.F. Berger. Applied Optimal Designs . Jhon Wiley \& Sons Ltd, Chichester, 2005. 19, 20

[MS16] Zerina Masetic e Abdulhamit Subasi. Congestive heart failure detection using random forest classifier. Computer Methods and Programs in Biomedicine, 130:5464, 7 2016. 15

[NC00] J. Shawe-Taylor N. Cristianini. Support Vector Machines. Em An Introduction to Support Vector Machines and Other Kernel-based Learning Methods, páginas 93-124. Cambridge University Press, London, 3 2000. 13

[NJHH14] Noman Naseer, Melissa Jiyoun Hong e Keum-Shik Hong. Online binary decision decoding using functional near-infrared spectroscopy for the development of brain-computer interface Keywords Binary decision decoding - Functional nearinfrared spectroscopy · Brain-computer interface · Yes/no decoding. Exp Brain Res, 232:555-564, 2014. 7

[NMB14] S C Nayak, B B Misra e H S Behera. Impact of Data Normalization on Stock Index Forecasting. International Journal of Computer Information Systems and Industrial Management Applications, 6:257-269, 2014. 31

[NS18] J. K. Nuamah e Younho Seong. Support vector machine (SVM) classification of cognitive tasks based on electroencephalography (EEG) engagement index. Brain-Computer Interfaces, 5(1):1-12, 1 2018. 41

[OOC86] Iain A D O'brien, Paul O'hare e Roger J M Corrall. Heart rate variability in healthy subjects: effect of age and the derivation of normal ranges for tests of autonomic function. Br Heart J, 55:348-54, 1986. 12, 26

$\left[\mathrm{PFMGO}^{+} 11\right]$ Fabian Pedregosa FABIANPEDREGOSA, Vincent Michel, Olivier Grisel OLIVIERGRISEL, Mathieu Blondel, Peter Prettenhofer, Ron Weiss, Jake Vanderplas, David Cournapeau, Fabian Pedregosa, Gaël Varoquaux, Alexandre Gramfort, Bertrand Thirion, Olivier Grisel, Vincent Dubourg, Alexandre Passos, Matthieu Brucher, Matthieu Perrot andÉdouardand, andÉdouard Duchesnay e FRÉdouard Duchesnay EDOUARDDUCHESNAY. Scikit-learn: Machine Learning in Python Gaël Varoquaux Bertrand Thirion Vincent Dubourg Alexandre Passos PEDREGOSA, VAROQUAUX, GRAMFORT ET AL. Matthieu Perrot. Relatório técnico, 2011. 15

[PG94] S. M. Pincus e A. L. Goldberger. Physiological time-series analysis: What does regularity quantify?, 1994. 12

[Por95] Stephen W. Porges. Cardiac vagal tone: A physiological index of stress. Neuroscience and Biobehavioral Reviews, 19(2):225-233, 1995. 11

[POS $\left.{ }^{+} 17\right]$ Joana Pereira, Patrick Ofner, Andreas Schwarz, Andreea Ioana Sburlea e Gernot R. Müller-Putz. EEG neural correlates of goal-directed movement intention. NeuroImage, 149:129-140, 4 2017. 7 
[PP17] Chinmay Pai e Kedar Potdar. A Comparative Study of Categorical Variable Encoding Techniques for Neural Network Classifiers. Article in International Journal of Computer Applications, 175(4):975-8887, 2017. 31

[Pra17] Joshi Prateek. Artificial Intelligence with Python. 2017. 15

$\left[\mathrm{PSA}^{+}\right.$17] Daniel J. Plews, Ben Scott, Marco Altini, Matt Wood, Andrew E. Kilding e Paul B. Laursen. Comparison of Heart-Rate-Variability Recording With Smartphone Photoplethysmography, Polar H7 Chest Strap, and Electrocardiography. International Journal of Sports Physiology and Performance, 12(10):1324-1328, 11 2017. xiii, 8

[PSBA $\left.{ }^{+} 12\right]$ Tobias Pistohl, Andreas Schulze-Bonhage, Ad Aertsen, Carsten Mehring e Tonio Ball. Decoding natural grasp types from human ECoG. NeuroImage, 59(1):248260, 1 2012. 7

[PTB14] Hyeong Dong Park e Catherine Tallon-Baudry. The neural subjective frame: From bodily signals to perceptual consciousness. Philosophical Transactions of the Royal Society B: Biological Sciences, 369(1641), 5 2014. 1

[PTU14] Rahul Pitale, Kapil Tajane e Jayant Umale. Heart Rate Variability Classification and Feature Extraction Using Support Vector Machine and PCA: An Overview. Relatório Técnico 1, 2014. 12

[PW00] Panos Y Papalambros e Douglass J Wilde. Principles of optimal design: modeling and computation. Cambridge university press, 2000. 20

[QH14] Daniel S. Quintana e James A. J. Heathers. Considerations in the assessment of heart rate variability in biobehavioral research. Frontiers in Psychology, 5:805, 7 2014. 12,25

$\left[\mathrm{RJO}^{+} 94\right]$ T.W. Robbins, M. James, A.M. Owen, B.J. Sahakian, L. McInnes e P. Rabbitt. Cambridge Neuropsychological Test Automated Battery (CANTAB): A Factor Analytic Study of a Large Sample of Normal Elderly Volunteers. Dementia and Geriatric Cognitive Disorders, 5(5):266-281, 1994. 6

$\left[\mathrm{RKG}^{+} 17\right]$ Timothy N. Rubin, Oluwasanmi Koyejo, Krzysztof J. Gorgolewski, Michael N. Jones, Russell A. Poldrack e Tal Yarkoni. Decoding brain activity using a large-scale probabilistic functional-anatomical atlas of human cognition. PLOS Computational Biology, 13(10):e1005649, 10 2017. 6

$\left[\mathrm{RLLV}^{+} 14\right]$ L. Rodríguez-Liñares, M.J. Lado, X.A. Vila, A.J. Méndez e P. Cuesta. gHRV: Heart rate variability analysis made easy. Computer Methods and Programs in Biomedicine, 116(1):26-38, 8 2014. 9, 24, 27, 29

$\left[\mathrm{RMF}^{+}\right.$16] Maria R Restivo, Margaret C McKinnon, Benicio N Frey, Geoffrey B Hall e Valerie $\mathrm{H}$ Taylor. Effect of obesity on cognition in adults with and without a mood disorder: study design and methods. BMJ open, 6(2):e009347, 2016. 6

$\left[\mathrm{RPR}^{+}\right]$Gil Rodas, Carles Pedret, Juan Ramos, Lluis Capdevila, Antoni Abad Mútua Activa e Gil FC Rodas Servicios Médicos Barcelona Avda Arístides Maillol. VARIABILIDAD DE LA FRECUENCIA CARDIACA: CONCEPTO, MEDIDAS Y RELACIÓN CON ASPECTOS CLÍNICOS (PARTE II) VARIABILIDAD DE LA FRECUENCIA CARDIACA: CONCEPTO, MEDIDAS Y RELACIÓN CON ASPECTOS CLÍNICOS (PARTE II)* HEART RATE VARIABILITY: DEFINITION, MEASUREMENT AN. Relatório técnico. 10 
[SE05] Abdulhamit Subasi e Ergun Erçelebi. Classification of EEG signals using neural network and logistic regression. Computer Methods and Programs in Biomedicine, 78(2):87-99, 5 2005. 14

$\left[\mathrm{SFC}^{+} 11\right]$ Thomas R. Shultz, Scott E. Fahlman, Susan Craw, Periklis Andritsos, Panayiotis Tsaparas, Ricardo Silva, Chris Drummond, Charles X. Ling, Victor S. Sheng, Chris Drummond, Pier Luca Lanzi, João Gama, R. Paul Wiegand, Prithviraj Sen, Galileo Namata, Mustafa Bilgic, Lise Getoor, Jun He, Sanjay Jain, Frank Stephan, Sanjay Jain, Frank Stephan, Claude Sammut, Michael Harries, Claude Sammut, Kai Ming Ting, Bernhard Pfahringer, John Case, Sanjay Jain, Kiri L. Wagstaff, Siegfried Nijssen, Anthony Wirth, Charles X. Ling, Victor S. Sheng, Xinhua Zhang, Claude Sammut, Nicola Cancedda, Jean-Michel Renders, Pietro Michelucci, Daniel Oblinger, Eamonn Keogh e Abdullah Mueen. Confusion Matrix. Em Encyclopedia of Machine Learning, páginas 209-209. Springer US, 2011. 15

[SG17] Fred Shaffer e J. P. Ginsberg. An Overview of Heart Rate Variability Metrics and Norms. Frontiers in Public Health, 5:258, 9 2017. 9, 11

[SH77] Philip H. Swain e Hans Hauska. DECISION TREE CLASSIFIER: DESIGN AND POTENTIAL. IEEE Trans Geosci Electron, GE-15(3):142-147, 1977. 14

[Sha15] Matthew Shardlow. An Analysis of Feature Selection Techniques. Relatório técnico, The University of Manchester, Manchester, 2015. 17

[SHY ${ }^{+}$09] Glenn E. Smith, Patricia Housen, Kristine Yaffe, Ronald Ruff, Robert F. Kennison, Henry W. Mahncke e Elizabeth M. Zelinski. A Cognitive Training Program Based on Principles of Brain Plasticity: Results from the Improvement in Memory with Plasticity-based Adaptive Cognitive Training (IMPACT) Study. Journal of the American Geriatrics Society, 57(4):594-603, 4 2009. 1

[SIL07] Y. Saeys, I. Inza e P. Larranaga. A review of feature selection techniques in bioinformatics. Bioinformatics, 23(19):2507-2517, 10 2007. 17

[SL05] Leif. Sornmo e Pablo Laguna. Bioelectrical signal processing in cardiac and neurological appli Elsevier Academic Press, 2005. 5

[Slo18] Scott D. Slotnick. Types of Memory and Brain Regions of Interest. Em Cognitive Neuroscience of Memory, páginas 1-23. Cambridge University Press, 5 2018. 7

[SMS ${ }^{+}$07] William W. Seeley, Vinod Menon, Alan F. Schatzberg, Jennifer Keller, Gary H. Glover, Heather Kenna, Allan L. Reiss e Michael D. Greicius. Dissociable intrinsic connectivity networks for salience processing and executive control. Journal of Neuroscience, 27(9):2349-2356, 2 2007. 1

[SMWY13] Jerritta Selvaraj, Murugappan Murugappan, Khairunizam Wan e Sazali Yaacob. Classification of emotional states from electrocardiogram signals: a non-linear approach based on Hurst. Biomedical engineering online, 12:44, 5 2013. 10

[Sod19] S. Soderi. Cybersecurity Assessment of the Polar Bluetooth Low Energy Heart-Rate Sensor. Em Lecture Notes of the Institute for Computer Sciences, Social-Informatics and Telecommunications Engineering, LNICST, volume 297 LNICST, páginas 252-265. Springer, 10 2019. 23

[SRR $\left.{ }^{+} 12\right]$ W. R. Shirer, S. Ryali, E. Rykhlevskaia, V. Menon e M. D. Greicius. Decoding subject-driven cognitive states with whole-brain connectivity patterns. Cerebral Cortex, 22(1):158-165, 1 2012. 1, 2, 7, 25, 41 
[SS20] Dalwinder Singh e Birmohan Singh. Investigating the impact of data normalization on classification performance. Applied Soft Computing, 97:105524, 122020. 31

[SSNM20] Kathryn E. Speer, Stuart Semple, Nenad Naumovski e Andrew J. McKune. Measuring Heart Rate Variability Using Commercially Available Devices in Healthy Children: A Validity and Reliability Study. European Journal of Investigation in Health, Psychology and Education, 10(1):390-404, 1 2020. 8

[SvMKK02] Hugo P Sondermeijer, Alexander G.J van Marle, Peter Kamen e Henry Krum. Acute effects of caffeine on heart rate variability. The American Journal of Cardiology, 90(8):906-907, 10 2002. 12, 41

[SVS04] D. Singh, K. Vinod e S. C. Saxena. Sampling frequency of the RR interval time series for spectral analysis of heart rate variability. Journal of Medical Engineering and Technology, 28(6):263-272, 11 2004. 27

[SYZ ${ }^{+}$17] Hongyu Shi, Licai Yang, Lulu Zhao, Zhonghua Su, Xueqin Mao, Li Zhang e Chengyu Liu. Differences of Heart Rate Variability Between Happiness and Sadness Emotion States: A Pilot Study. Journal of Medical and Biological Engineering, 37(4), 2017. 41

[Szt04] Juan Sztajzel. Heart rate variability: a noninvasive electrocardiographic method to measure the autonomic nervous system. Relatório técnico, 2004. 9

[TG13] Tsung-han Tsai e Jeff Gill. Interactions in generalized linear models: theoretical issues and an application to personal vote-earning attributes. Social Sciences, 2(2):91-113, 2013. 21

[Tha13] Nitish V. Thakor. Translating the brain-machine interface, 11 2013. 1

[TLJBE07] Töres Theorell, Yvonne Liljeholm-Johansson, Helena Björk e Mats Ericson. Saliva testosterone and heart rate variability in the professional symphony orchestra after "public faintings" of an orchestra member. Psychoneuroendocrinology, 32(6):660-668, 7 2007. 11

[USMA98] Ken Umetani, Donald H Singer, Rollin McCraty e Mike Atkinson. Twenty-Four Hour Time Domain Heart Rate Variability and Heart Rate: Relations to Age and Gender Over Nine Decades. Journal of the American College of Cardiology, 31(3):593-601, 3 1998. 11

$\left[\mathrm{VSP}^{+}\right.$08] L.C.M. Vanderlei, R.A. Silva, C.M. Pastre, F.M. Azevedo e M.F. Godoy. Comparison of the Polar S810i monitor and the ECG for the analysis of heart rate variability in the time and frequency domains. Brazilian Journal of Medical and Biological Research, 41(10):854-859, 9 2008. 8

[WA14] Steven A Weissman e Neal G Anderson. Design of Experiments (DoE) and Process Optimization. A Review of Recent Publications. 2014. 18

[WDS $\left.{ }^{+} 11\right]$ Wei Wang, Alan D. Degenhart, Gustavo P. Sudre, Dean A. Pomerleau e Elizabeth C. Tyler-Kabara. Decoding semantic information from human electrocorticographic (ECoG) signals. Em Proceedings of the Annual International Conference of the IEEE Engineering in Medicine and Biology Society, EMBS, páginas 62946298, 2011. 41

[Wel67] Peter D. Welch. The Use of Fast Fourier Transform for the Estimation of Power Spectra: A Method Based on Time Averaging Over Short, Modified Periodograms. IEEE Transactions on Audio and Electroacoustics, 15(2):70-73, 1967. 26 